CERN-PPE/95-075

18 May 1995

\title{
A model independent measurement of quark and gluon jet properties and differences
}

\author{
The OPAL Collaboration
}

\begin{abstract}
Three jet events are selected from hadronic $Z^{0}$ decays with a symmetry such that the two lower energy jets are produced with the same energy and in the same jet environment. In some of the events, a displaced secondary vertex is reconstructed in one of the two lower energy jets, which permits the other lower energy jet to be identified as a gluon jet, with an estimated purity of about $93 \%$. Comparing these gluon jets to the inclusive sample of lower energy jets from the symmetric data set yields direct, model independent measurements of quark and gluon jet properties, which have essentially no bias except from the jet definition. Results are reported using both the $k_{\perp}$ and cone jet definitions. For the $k_{\perp}$ algorithm, we find

$$
\left.\frac{\left\langle n_{k_{\perp}}^{\text {ch. }}\right\rangle_{\text {gluon }}}{\left\langle n_{k_{\perp}}^{\text {ch. }}\right\rangle_{\text {quark }}}=1.25 \pm 0.02 \text { (stat. }\right) \pm 0.03 \text { (syst.) }
$$

for the ratio of the mean charged particle multiplicity of gluon to quark jets, while for the cone algorithm, we find

$$
\frac{\left\langle n_{\text {cone }}^{\text {ch. }}\right\rangle_{\text {gluon }}}{\left\langle n_{\text {cone }}^{\text {ch. }}\right\rangle_{\text {quark }}}=1.10 \pm 0.02 \text { (stat.) } \pm 0.02 \text { (syst.) }
$$

using a cone size of $30^{\circ}$. We also report measurements of the angular distributions of particle energy and multiplicity around the jet directions, and of the fragmentation functions of the jets. Gluon jets are found to be substantially broader and to have a markedly softer fragmentation function than quark jets, in agreement with our earlier observations.
\end{abstract}

(To be submitted to Zeitschrift für Physik C) 


\section{The OPAL Collaboration}

R. Akers ${ }^{16}$, G. Alexander ${ }^{23}$, J.Allison ${ }^{16}$, N. Altekamp ${ }^{5}$, K. Ametewee ${ }^{25}$, K.J. Anderson ${ }^{9}$, S. Anderson ${ }^{12}$, S. Arcelli ${ }^{2}$, S. Asai ${ }^{24}$, D. Axen ${ }^{29}$, G. Azuelos ${ }^{18, a}$, A.H. Balli ${ }^{17}$ E. Barberio ${ }^{26}$, R.J. Barlow ${ }^{16}$, R. Bartoldus ${ }^{3}$, J.R. Batley ${ }^{5}$, G.Beaudoin ${ }^{18}$, J. Bechtluft ${ }^{14}$, A. Beck ${ }^{23}$, G.A.Beck ${ }^{13}$,

C.Beeston ${ }^{16}$, T.Behnke ${ }^{27}$, K.W.Bell ${ }^{20}$, G. Bella ${ }^{23}$, S. Bentvelsen ${ }^{8}$, P. Berlich ${ }^{10}$, S. Bethke $^{14}$, O. Biebel ${ }^{14}$, I.J.Bloodworth ${ }^{1}$, P. Bock ${ }^{11}$, H.M.Bosch ${ }^{11}$, M. Boutemeur ${ }^{18}$, S. Braibant ${ }^{12}$, P. Bright-Thomas ${ }^{25}$, R.M. Brown ${ }^{20}$, A. Buijs ${ }^{8}$, H.J.Burckhart ${ }^{8}$, C. Burgard ${ }^{27}$, R. Bürgin ${ }^{10}$, P. Capiluppi ${ }^{2}$, R.K. Carnegie ${ }^{6}$, A.A. Carter ${ }^{13}$, J.R. Carter $^{5}$, C.Y.Chang ${ }^{17}$, C. Charlesworth ${ }^{6}$, D.G. Charlton ${ }^{1, b}$, S.L. Chu ${ }^{4}$, P.E.L.Clarke ${ }^{15}$, J.C.Clayton ${ }^{1}$, S.G. Clowes ${ }^{16}$, I. Cohen $^{23}$, J.E. Conboy ${ }^{15}$, O.C.Cooke ${ }^{16}$, M.Cuffiani ${ }^{2}$, S.Dado ${ }^{22}$, C.Dallapiccola ${ }^{17}$, G.M.Dallavalle ${ }^{2}$, C.Darling ${ }^{31}$, S. De Jong ${ }^{12}$, L.A.del Pozo ${ }^{8}$, H.Deng ${ }^{17}$, M.S. Dixit ${ }^{7}$, E. do Couto e Silva ${ }^{12}$, J.E. Duboscq ${ }^{8}$, E. Duchovni ${ }^{26}$, G. Duckeck ${ }^{8}$, I.P.Duerdoth ${ }^{16}$, U.C.Dunwoody ${ }^{8}$, J.E.G.Edwards ${ }^{16}$, P.G.Estabrooks ${ }^{6}$, H.G.Evans ${ }^{9}$,

F. Fabbri ${ }^{2}$, B.Fabbro ${ }^{21}$, M.Fanti ${ }^{2}$, P. Fath ${ }^{11}$, F.Fiedler ${ }^{12}$, M.Fierro ${ }^{2}$, M.Fincke-Keeler ${ }^{28}$, H.M. Fischer ${ }^{3}$, R. Folman ${ }^{26}$, D.G.Fong ${ }^{17}$, M.Foucher ${ }^{17}$, H. Fukui ${ }^{24}$, A.Fürtjes ${ }^{8}$, P. Gagnon ${ }^{6}$, A. Gaidot ${ }^{21}$, J.W. Gary ${ }^{4}$, J. Gascon ${ }^{18}$, S.M. Gascon-Shotkin ${ }^{17}$, N.I. Geddes ${ }^{20}$, C. Geich-Gimbel ${ }^{3}$, S.W. Gensler ${ }^{9}$, F.X. Gentit ${ }^{21}$, T. Geralis ${ }^{20}$, G. Giacomelli ${ }^{2}$, P. Giacomelli ${ }^{4}$, R. Giacomelli², V. Gibson ${ }^{5}$, W.R. Gibson ${ }^{13}$, J.D. Gillies ${ }^{20}$, D.M. Gingrich ${ }^{30, a}$, J. Goldberg ${ }^{22}$, M.J. Goodrick ${ }^{5}$, W. Gorn ${ }^{4}$,

C. Grandi ${ }^{2}$, E. Gross ${ }^{26}$, G.G.Hanson ${ }^{12}$, M.Hansroul ${ }^{8}$, M. Hapke ${ }^{13}$, C.K. Hargrove ${ }^{7}$, P.A.Hart ${ }^{9}$, C.Hartmann ${ }^{3}$, M.Hauschild ${ }^{8}$, C.M.Hawkes ${ }^{8}$, R.Hawkings ${ }^{8}$, R.J.Hemingway ${ }^{6}$, G. Herten ${ }^{10}$, R.D.Heuer ${ }^{8}$, J.C.Hill ${ }^{5}$, S.J. Hillier ${ }^{8}$, T.Hilse ${ }^{10}$, P.R.Hobson ${ }^{25}$, D. Hochman ${ }^{26}$, R.J. Homer ${ }^{1}$,

A.K.Honma ${ }^{28, a}$, R. Howard ${ }^{29}$, R.E.Hughes-Jones ${ }^{16}$, D.E. Hutchcroft ${ }^{5}$, P. Igo-Kemenes ${ }^{11}$,

D.C. Imrie ${ }^{25}$, A. Jawahery ${ }^{17}$, P.W. Jeffreys ${ }^{20}$, H. Jeremie ${ }^{18}$, M. Jimack ${ }^{1}$, A. Joly ${ }^{18}$, M. Jones ${ }^{6}$, R.W.L. Jones ${ }^{8}$, P. Jovanovic ${ }^{1}$, J. Kanzaki ${ }^{24}$, D. Karlen ${ }^{6}$, K. Kawagoe ${ }^{24}$, T. Kawamoto ${ }^{24}$, R.K.Keeler ${ }^{28}$,

R.G.Kellogg ${ }^{17}$, B.W.Kennedy ${ }^{20}$, B.J.King ${ }^{8}$, J.King ${ }^{13}$, J. Kirk ${ }^{29}$, S. Kluth ${ }^{5}$, T.Kobayashi ${ }^{24}$, M.Kobel ${ }^{10}$, D.S. Koetke ${ }^{6}$, T.P. Kokott ${ }^{3}$, S. Komamiya ${ }^{24}$, R.Kowalewski ${ }^{8}$, T.Kress ${ }^{11}$, P. Krieger ${ }^{6}$,

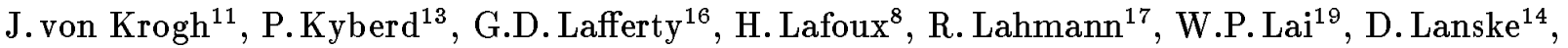

J. Lauber ${ }^{8}$, J.G. Layter ${ }^{4}$, A.M.Lee ${ }^{31}$,E. Lefebvre ${ }^{18}$, D. Lellouch ${ }^{26}$, J. Letts ${ }^{2}$, L. Levinson ${ }^{26}$, S.L. Lloyd ${ }^{13}$, F.K. Loebinger ${ }^{16}$, G.D.Long ${ }^{17}$, B. Lorazo $^{18}$, M.J.Losty ${ }^{7}$, J. Ludwig ${ }^{10}$, A. Luig ${ }^{10}$, A. Malik ${ }^{21}$, M.Mannelli ${ }^{8}$, S. Marcellini ${ }^{2}$, C.Markus ${ }^{3}$, A.J. Martin ${ }^{13}$, J.P. Martin ${ }^{18}$, T. Mashimo ${ }^{24}$,

W.Matthews ${ }^{25}$, P.Mättig ${ }^{3}$, J.McKenna ${ }^{29}$, E.A.Mckigney ${ }^{15}$, T.J.McMahon ${ }^{1}$, A.I.McNab ${ }^{13}$, F.Meijers ${ }^{8}$, S.Menke ${ }^{3}$, F.S.Merritt ${ }^{9}$, H. Mes $^{7}$, A.Michelini ${ }^{8}$, G. Mikenberg ${ }^{26}$, D.J. Miller ${ }^{15}$, R. Mir $^{26}$, W.Mohr ${ }^{10}$, A.Montanari ${ }^{2}$, T. Mori ${ }^{24}$, M.Morii ${ }^{24}$, U. Müller ${ }^{3}$, B. Nellen ${ }^{3}$, B. Nijjhar ${ }^{16}$, S.W.O'Neale ${ }^{1}$, F.G.Oakham ${ }^{7}$, F. Odorici ${ }^{2}$, H.O. Ogren ${ }^{12}$, N.J. Oldershaw ${ }^{16}$, C.J. Oram ${ }^{28, a}$, M.J.Oreglia ${ }^{9}$, S. Orito ${ }^{24}$, F.Palmonari ${ }^{2}$, J.P.Pansart ${ }^{21}$, G.N.Patrick ${ }^{20}$, M.J.Pearce ${ }^{1}$, P.D.Phillips ${ }^{16}$, J.E.Pilcher ${ }^{9}$, J.Pinfold ${ }^{30}$,

D.E.Plane ${ }^{8}$, P.Poffenberger ${ }^{28}$, B.Poli ${ }^{2}$, A.Posthaus ${ }^{3}$, T.W.Pritchard ${ }^{13}$, H.Przysiezniak ${ }^{30}$,

M.W.Redmond ${ }^{8}$, D.L. Rees ${ }^{1}$, D. Rigby ${ }^{1}$, M.G.Rison ${ }^{5}$, S.A.Robins ${ }^{13}$, N. Rodning ${ }^{30}$, J.M. Roney ${ }^{28}$,

E. $\operatorname{Ros}^{8}$, A.M.Rossi ${ }^{2}$, M.Rosvick ${ }^{28}$, P. Routenburg ${ }^{30}$, Y.Rozen ${ }^{8}$, K. Runge ${ }^{10}$, O. Runolfsson ${ }^{8}$,

D.R.Rust ${ }^{12}$, M.Sasaki ${ }^{24}$, C.Sbarra ${ }^{2}$, A.D.Schaile ${ }^{8}$, O.Schaile ${ }^{10}$, F. Scharf ${ }^{3}$, P.Scharff-Hansen ${ }^{8}$, P.Schenk ${ }^{4}$, B.Schmitt ${ }^{3}$, M.Schröder ${ }^{8}$, H.C.Schultz-Coulon ${ }^{10}$, M.Schulz ${ }^{8}$, P.Schütz ${ }^{3}$, J.Schwiening ${ }^{3}$,

W.G.Scott ${ }^{20}$, M.Settles ${ }^{12}$, T.G.Shears ${ }^{16}$, B.C.Shen ${ }^{4}$, C.H. Shepherd-Themistocleous ${ }^{7}$,

P. Sherwood ${ }^{15}$, G.P.Siroli ${ }^{2}$, A.Skillman ${ }^{15}$, A.Skuja ${ }^{17}$, A.M.Smith ${ }^{8}$, T.J. Smith ${ }^{28}$, G.A.Snow ${ }^{17}$,

R. Sobie $^{28}$, S.Söldner-Rembold ${ }^{10}$, R.W.Springer ${ }^{30}$, M.Sproston ${ }^{20}$, A.Stahl ${ }^{3}$, M.Starks ${ }^{12}$,

C.Stegmann ${ }^{10}$, K.Stephens ${ }^{16}$, J. Steuerer ${ }^{28}$, B.Stockhausen ${ }^{3}$, D. Strom ${ }^{19}$, P.Szymanski ${ }^{20}$,

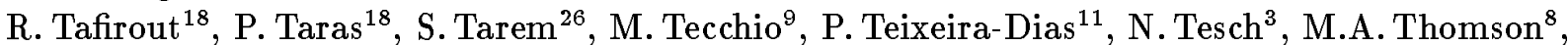

E. von Törne ${ }^{3}$, S. Towers ${ }^{6}$, M. Tscheulin ${ }^{10}$, T. Tsukamoto ${ }^{24}$, A.S. Turcot ${ }^{9}$, M.F. Turner-Watson ${ }^{8}$, P. Utzat ${ }^{11}$, R. Van Kooten ${ }^{12}$, G. Vasseur ${ }^{21}$, P. Vikas ${ }^{18}$, M. Vincter ${ }^{28}$, F. Wäckerle ${ }^{10}$, A. Wagner ${ }^{27}$,

D.L. Wagner ${ }^{9}$, C.P. Ward $^{5}$, D.R. Ward ${ }^{5}$, J.J. Ward ${ }^{15}$, P.M. Watkins ${ }^{1}$, A.T. Watson ${ }^{1}$, N.K. Watson ${ }^{7}$,

P. Weber ${ }^{6}$, P.S. Wells ${ }^{8}$, N. Wermes ${ }^{3}$, B. Wilkens ${ }^{10}$, G.W.Wilson ${ }^{27}$, J.A. Wilson ${ }^{1}$, T. Wlodek ${ }^{26}$,

G. Wolf ${ }^{26}$, S. Wotton ${ }^{11}$, T.R. Wyatt ${ }^{16}$, G. Yekutieli ${ }^{26}$, V.Zacek ${ }^{18}$, W.Zeuner ${ }^{8}$, G.T.Zorn ${ }^{17}$. 
${ }^{1}$ School of Physics and Space Research, University of Birmingham, Birmingham B15 2TT, UK

${ }^{2}$ Dipartimento di Fisica dell' Università di Bologna and INFN, I-40126 Bologna, Italy

${ }^{3}$ Physikalisches Institut, Universität Bonn, D-53115 Bonn, Germany

${ }^{4}$ Department of Physics, University of California, Riverside CA 92521, USA

${ }^{5}$ Cavendish Laboratory, Cambridge CB3 0HE, UK

${ }^{6}$ Carleton University, Department of Physics, Colonel By Drive, Ottawa, Ontario K1S 5B6, Canada

${ }^{7}$ Centre for Research in Particle Physics, Carleton University, Ottawa, Ontario K1S 5B6, Canada

${ }^{8}$ CERN, European Organisation for Particle Physics, CH-1211 Geneva 23, Switzerland

${ }^{9}$ Enrico Fermi Institute and Department of Physics, University of Chicago, Chicago IL 60637, USA

${ }^{10}$ Fakultät für Physik, Albert Ludwigs Universität, D-79104 Freiburg, Germany

${ }^{11}$ Physikalisches Institut, Universität Heidelberg, D-69120 Heidelberg, Germany

${ }^{12}$ Indiana University, Department of Physics, Swain Hall West 117, Bloomington IN 47405, USA

${ }^{13}$ Queen Mary and Westfield College, University of London, London E1 4NS, UK

${ }^{14}$ Technische Hochschule Aachen, III Physikalisches Institut, Sommerfeldstrasse 26-28, D-52056 Aachen, Germany

${ }^{15}$ University College London, London WC1E 6BT, UK

${ }^{16}$ Department of Physics, Schuster Laboratory, The University, Manchester M13 9PL, UK

${ }^{17}$ Department of Physics, University of Maryland, College Park, MD 20742, USA

${ }^{18}$ Laboratoire de Physique Nucléaire, Université de Montréal, Montréal, Quebec H3C 3J7, Canada

${ }^{19}$ University of Oregon, Department of Physics, Eugene OR 97403, USA

${ }^{20}$ Rutherford Appleton Laboratory, Chilton, Didcot, Oxfordshire OX11 0QX, UK

${ }^{21} \mathrm{CEA}$, DAPNIA/SPP, CE-Saclay, F-91191 Gif-sur-Yvette, France

${ }^{22}$ Department of Physics, Technion-Israel Institute of Technology, Haifa 32000, Israel

${ }^{23}$ Department of Physics and Astronomy, Tel Aviv University, Tel Aviv 69978, Israel

${ }^{24}$ International Centre for Elementary Particle Physics and Department of Physics, University of Tokyo, Tokyo 113, and Kobe University, Kobe 657, Japan

${ }^{25}$ Brunel University, Uxbridge, Middlesex UB8 3PH, UK

${ }^{26}$ Particle Physics Department, Weizmann Institute of Science, Rehovot 76100, Israel

${ }^{27}$ Universität Hamburg/DESY, II Institut für Experimental Physik, Notkestrasse 85, D-22607 Hamburg, Germany

${ }^{28}$ University of Victoria, Department of Physics, P O Box 3055, Victoria BC V8W 3P6, Canada

${ }^{29}$ University of British Columbia, Department of Physics, Vancouver BC V6T 1Z1, Canada

${ }^{30}$ University of Alberta, Department of Physics, Edmonton AB T6G 2J1, Canada

${ }^{31}$ Duke University, Department of Physics, Durham, NC 27708-0305, USA

${ }^{a}$ Also at TRIUMF, Vancouver, Canada V6T $2 \mathrm{~A} 3$

${ }^{b}$ Royal Society University Research Fellow 


\section{Introduction}

The gauge theory of strong interactions, Quantum Chromodynamics (QCD), predicts large differences between the properties of quark- and gluon-induced jets [1]. These differences are a consequence of the different probabilities for a quark or gluon to radiate an additional gluon. For quark and gluon jets of equal energy produced under the same circumstances, the mean particle multiplicity of a gluon jet is predicted to be larger than that of a quark jet and thus its particle energy spectrum is predicted to be softer. Assuming the transverse energy scale of quark and gluon jet development to be about the same, the softer energy spectrum of the gluon jet implies that its particle multiplicity and energy should be distributed at larger mean angles with respect to the jet axis than is the case for quark jets. Many attempts have been made to observe such features in the data [2], but these studies often yielded inconclusive results either because the jets could not be selected without biasing them or because the analysis technique was not sensitive to quark-gluon jet differences.

In several earlier publications [3, 4], we introduced a method of comparing the properties of quark- and gluon-induced jets in an essentially unbiased and model independent manner. These studies resulted in an unambiguous confirmation of the predictions for quark and gluon jet differences given above. Three-jet events from hadronic $Z^{0}$ decays were selected with a onefold symmetry, namely such that the two lower energy jets, one of which was assumed to be a quark jet and the other a gluon jet, were both produced at an angle of about $150^{\circ}$ with respect to the highest energy jet. In some cases, one of the two lower energy jets could be identified as a quark jet due to the presence of a displaced secondary vertex. The lower energy jet without the displaced secondary vertex was thereby identified as a gluon jet using an anti-tagging method. The properties of these anti-tagged gluon jets were compared to those of the two lower energy jets from the inclusive one-fold symmetric sample. The essential feature of our analysis which allowed an unambiguous interpretation of the results was that the quark and gluon jets being compared had the same energy and were produced in an identical jet environment because of the symmetric event geometry. There was thus no need to employ Monte Carlo simulations to account for kinematic differences between the quark and gluon jet samples, in contrast to some earlier studies of quark and gluon jet differences. Although model independent, some quantitative aspects of the analysis which were sensitive to the treatment of soft particles, such as the ratio of the mean particle multiplicity values between gluon and quark jets, were found to depend on the jet definition.

Our earlier work [4] was based on the $k_{\perp}$ jet finder [5], which is an extension of the invariant mass jet algorithms commonly used by $\mathrm{e}^{+} \mathrm{e}^{-}$experiments. With $\mathrm{p} \overline{\mathrm{p}}$ experiments, it is more common to define jets using the cone jet finder [6]. The use of different jet definitions by $\mathrm{e}^{+} \mathrm{e}^{-}$ and $\mathrm{p} \overline{\mathrm{p}}$ experiments has made it difficult to compare the properties of jets produced in the two different processes. First results on the use of the cone jet finder with our $\mathrm{e}^{+} \mathrm{e}^{-}$data were recently reported in [7].

In this paper, we extend our study of quark and gluon jet differences by employing a cone definition for the jets. An update and extension of our earlier work based on the $k_{\perp}$ jet definition is also included. The data sample, collected with the OPAL detector at the $\mathrm{e}^{+} \mathrm{e}^{-}$collider LEP at CERN, is about three times larger than that used for our earlier studies. Results obtained using the cone algorithm, in conjunction with those obtained using the $k_{\perp}$ algorithm, provide 
a means to establish the sensitivity of the observed quark and gluon jet characteristics to the jet definition. Use of the cone algorithm is also expected to facilitate the comparison of our data with jet data from $\mathrm{p} \overline{\mathrm{p}}$ experiments, as noted above. In our earlier publications, the data were not corrected for detector acceptance and resolution. In the present study, the data are corrected for these effects.

In section 2, we present a brief account of the OPAL detector and the multihadron event selection procedure. The three jet event definition is discussed in section 3 . In sections 4 and 5, the analysis method for obtaining fully corrected information for the separated quark and gluon jet samples is described. The results obtained with the $k_{\perp}$ and cone jet finders are given in sections 6 and 7. Systematic uncertainties of the measurements are discussed in section 8 . Section 9 presents a comparison of the results found in this paper for the gluon jet fragmentation function to those found using our measurements of the longitudinal and transverse fragmentation functions, $F_{L}(x)$ and $F_{T}(x)$ [8]. Section 10 contains a discussion and summary.

\section{Detector and data selection}

The OPAL detector is described in detail elsewhere [9]. A brief account of those features most relevant to the present study is given below.

Charged particle trajectories are reconstructed using a central detector, which includes three systems of drift chambers: an inner vertex chamber, a large volume jet chamber and specialized chambers at the outer radius of the jet chamber which improve the measurements in the $z$ direction. ${ }^{1}$ The central detector also includes a silicon microvertex detector, discussed below. The tracking chambers are enclosed by a solenoidal magnet coil which provides an axial field of approximately $0.435 \mathrm{~T}$. The most important tracking detector for the momentum measurement is the jet chamber, which provides up to 159 space-points per track and nearly $100 \%$ track finding efficiency in the region $|\cos \theta|<0.95$. The momentum resolution for charged tracks in the plane perpendicular to the beam axis is $\sigma_{p_{\perp}} / p_{\perp} \approx \sqrt{(0.020)^{2}+\left(0.0015 \cdot p_{\perp} /[\mathrm{GeV} / c]\right)^{2}}$. The average angular resolution is about $0.1 \mathrm{mrad}$ in $\phi$ and $1 \mathrm{mrad}$ in $\theta$.

Of particular importance to this analysis is the silicon microvertex detector [10]. This device contains two layers of silicon microstrip detectors, one positioned at a radius of $6.1 \mathrm{~cm}$ with an angular coverage of $|\cos \theta|<0.83$ and the other positioned at a radius of $7.5 \mathrm{~cm}$ with a coverage of $|\cos \theta|<0.77$. A position resolution of about $10 \mu \mathrm{m}$ is currently achieved with this detector in the plane perpendicular to the beam axis, with an efficiency of about $95 \%$ for finding silicon detector $r$ - $\phi$ information in either layer for tracks in hadronic events which pass through the active region.

Electromagnetic energy is measured by a lead-glass calorimeter located outside the magnet coil, with a barrel $(|\cos \theta|<0.82)$ and two endcap $(0.81<|\cos \theta|<0.98)$ sections. Each lead glass block subtends approximately $40 \times 40 \mathrm{mrad}^{2}$. The depth of material to the back

\footnotetext{
${ }^{1}$ Our coordinate system is defined so that $z$ is the coordinate parallel to the $\mathrm{e}^{-}$beam axis, $r$ is the coordinate normal to the beam axis, $\phi$ is the azimuthal angle around the beam axis and $\theta$ is the polar angle with respect to $z$.
} 
of the calorimeter is about 25 radiation lengths. The basic calorimeter entity is a cluster of energy, i.e. a group of contiguous blocks containing significant energy deposition. To minimize double counting of energy, clusters are used in this analysis only if they are not associated with a charged track. A cluster is associated with a charged track if the extrapolated track coordinates at the entrance to the calorimeter match to better than $80 \mathrm{mrad}$ in $\phi$ and $150 \mathrm{mrad}$ in $\theta$, if the cluster is in the barrel, or $50 \mathrm{mrad}$ in both $\phi$ and $\theta$, if it is in the endcap.

The analysis presented here is based on a sample of about 2800000 hadronic events collected at $\mathrm{e}^{+} \mathrm{e}^{-}$center-of-mass energies within $250 \mathrm{MeV}$ of the $\mathrm{Z}^{0}$ peak by the OPAL detector from 1991 to 1994. Specifications of the OPAL trigger are given in [11]. The procedures for identifying hadronic events are discussed in [12]. Within the geometrical region used for the present study, the efficiency of this selection is estimated to be greater than $99.6 \%$. Charged tracks used in this analysis were required to have at least 20 measured points in the jet chamber, to have a transverse momentum in the $r-\phi$ plane, $p_{\perp}$, greater than $0.15 \mathrm{GeV} / c$, to lie in the region $|\cos \theta|<0.94$ and to point to the origin to within $5 \mathrm{~cm}$ in the $r-\phi$ plane. In addition, they were required to yield a $\chi^{2}$ per degree-of-freedom of less than 100 for the track fit in the $r$ - $\phi$ plane. Clusters were required to be spread over at least two lead glass blocks and to have an energy greater than $0.1 \mathrm{GeV}$ if they were in the barrel section of the detector or greater than $0.3 \mathrm{GeV}$ if they were in the endcap section. For the present analysis, each accepted track and unassociated cluster was considered to be a particle. Tracks were assigned the pion mass. Clusters were assigned zero mass since they originate mostly from photons. Event cuts were applied to eliminate residual background and events in which a significant number of particles were lost near the beam direction. First, the number of accepted charged tracks was required to be at least five. Second, the thrust axis of the event was calculated using the particles and was required to satisfy $\left|\cos \left(\theta_{\text {thrust }}\right)\right|<0.9$. The residual background from all sources was estimated to be less than $1 \%$.

\section{$3 \quad$ Three-jet event selection}

One of the principal purposes of the present study is to investigate the properties of separated quark and gluon jet samples using different jet definitions. The jet definitions we select are based on the $k_{\perp}[5]$ and cone $[6,7]$ algorithms. We also study the sensitivity of the results to the choice of the resolution parameters for these two jet finding methods.

The $k_{\perp}$ jet finder begins by assigning each particle to be an individual jet. The scaled quantity $y_{i j}$ is then calculated for each jet pair:

$$
y_{i j}=\frac{2 \cdot \min \left(E_{i}^{2}, E_{j}^{2}\right) \cdot\left(1-\cos \theta_{i j}\right)}{E_{\mathrm{vis}}^{2}}
$$

where $E_{\text {vis. }}$ is the sum of the particle energies, $E_{i}$ is the energy of jet $i$ and $\theta_{i j}$ is the angle between jets $i$ and $j$. The pair with smallest $y_{i j}$ is combined into a single jet, $k$, where the momentum vector $\vec{p}_{k}$ and energy $E_{k}$ of the new jet are given by $\vec{p}_{k}=\vec{p}_{i}+\vec{p}_{j}$ and $E_{k}=E_{i}+E_{j}$. The process is repeated until all jet pairs have a $y_{i j}$ value which exceeds a resolution parameter,

$y_{c u t}$. The $y_{\text {cut }}$ value chosen as the standard for this analysis is 0.02 , which maximizes the event statistics for the event configuration we study. 
The cone jet finder begins by treating each particle in an event in turn as the axis of a cone of half angle $R$. The momenta of particles lying within this cone are summed. If the momentum sum and cone axis do not coincide, the momentum sum is taken to define a new cone axis and the process is iterated. When a stable solution is found, the particles in the cone are deemed to constitute a "proto-jet". If two narrow jets are separated by an angle between $R$ and $2 R$, they could be found as two separate proto-jets at this stage. To permit this configuration to be found as a single jet, the direction which bisects the angle between the two jet axes is also considered as a cone axis, and an iterative search for a stable proto-jet is performed. Proto-jets which are not identical to a previously found proto-jet and for which the total particle energy exceeds a minimum given by a cutoff $\epsilon$ are accepted as jets. If two jets overlap because they contain particles in common, an overlap fraction $f$ is defined by the ratio of the total energy of the particles in common to the energy of the lower energy jet. If $f$ exceeds 0.75 , the particles in common are assigned to the higher energy jet and the lower energy jet is eliminated. If $f$ is less than 0.75 , each particle in the overlap region is assigned to the jet to which it lies nearest in angle. The cone jet finder has two resolution parameters, $R$ and $\epsilon$ : the standard values we employ for this analysis are $30^{\circ}$ and $10 \mathrm{GeV}$, respectively. This value of $R$ is a natural choice for the events we study, as explained below, while this value of $\epsilon$ maximizes the event statistics for the choice of $R$.

With the cone jet finder, soft particles between jets often lie outside the cones and are not assigned to a jet. This represents an important difference compared to the $k_{\perp}$ jet finder. In the latter case, all particles in an event are associated with a jet, even those at large angles to the axes.

Events reconstructed with exactly three jets, using either the $k_{\perp}$ or cone method, are retained for further study. These events are interpreted as arising from two quark jets and a gluon jet. ${ }^{2}$ Each jet is required to contain at least two particles and to lie in the polar angle region $|\cos \theta|<0.9$. The sum of the angles between the three jets is required to exceed $358^{\circ}$ to eliminate non-planar events. The jets in each event are assigned a calculated energy based on the angles between them, assuming massless kinematics and perfect event reconstruction. One-fold symmetric three-jet events are selected by projecting the jets into the three-jet event plane and requiring the angles between the jet with the highest calculated energy and each of the two others to be $150 \pm 10^{\circ}$. A value of $150^{\circ}$ is chosen because this leads to well separated jets, good event statistics, and events in which the highest energy jets are virtually always quark jets, as discussed below. Thus the angle between the two lower energy jets is about $60^{\circ}$, which is why a value of $30^{\circ}$ for the cone size $R$ is a natural choice for our analysis. The event plane is defined by the plane normal to the smallest sphericity [13] eigenvector. In total, 65148 symmetric three-jet events are found using the $k_{\perp}$ jet finder, and 51317 using the cone jet finder. We refer to these events as the "normal-mixture" samples because they contain approximately the same composition of quark flavors and decays as the inclusive sample of on-peak hadronic $\mathrm{Z}^{0}$ events. ${ }^{3}$ About $65 \%$ of the events found using the cone algorithm are also found by the $k_{\perp}$ one. For the $k_{\perp}$ jet finder, the mean calculated jet energies are $42.43 \pm 0.01 \mathrm{GeV}$ for the highest energy jet and $24.39 \pm 0.01 \mathrm{GeV}$ for the two lower energy jets, while the mean visible jet energies, defined by summing the particle energies assigned to

\footnotetext{
${ }^{2}$ We do not distinguish between a quark and an anti-quark jet.

${ }^{3}$ Due to quark mass effects, there are predicted to be $2-4 \%$ fewer b quark events in the normal-mixture samples than in the inclusive multihadronic one, but otherwise the flavor composition of the two samples is believed to be the same.
} 
each jet, are $33.65 \pm 0.03 \mathrm{GeV}$ and $21.59 \pm 0.01 \mathrm{GeV}$, respectively. The corresponding values for the cone jet finder are $42.40 \pm 0.01 \mathrm{GeV}$ and $24.40 \pm 0.01 \mathrm{GeV}$ for the calculated energies and $31.31 \pm 0.03 \mathrm{GeV}$ and $19.79 \pm 0.02 \mathrm{GeV}$ for the visible ones. The visible energies obtained using the cone algorithm are somewhat smaller than those obtained using the $k_{\perp}$ algorithm since not all particles are assigned to a jet, as noted above. Since the highest energy jets have a much larger energy than the two lower energy ones, they are likely to be quark jets with high probability, due to the nature of the gluon radiation spectrum. From Monte Carlo study, this probability is estimated to be about $97 \%$. In this paper, jet energies refer to the calculated energies, and not to the visible ones, unless otherwise stated.

\section{$4 \quad$ Jet tagging using secondary vertices}

The method we introduced [4] for obtaining quark and gluon jet properties is based on the reconstruction of a displaced secondary vertex in one of the two lower energy jets of the normalmixture events. The secondary vertex is associated with heavy quark decay, especially that of the $b$ quark. At LEP, $b$ quarks are produced almost exclusively at the electro-weak vertex, directly from the $\mathrm{Z}^{0}$ decay: thus a jet with a b quark is likely to be a quark jet. The lower energy jets without secondary vertices thus constitute a highly enriched sample of gluon jets. By employing these "anti-tagged" gluon jets, we obtain jet properties which are virtually unbiased by the requirement that a reconstructed secondary vertex be present in an event.

To identify secondary vertices in jets, tracks in a jet which have coordinate information in $r$ - $\phi$ from at least one of the silicon detector layers are fitted to a common vertex. The track which contributes the largest $\chi^{2}$ value to the fit is removed if that $\chi^{2}$ is larger than 4.0. The fit is then repeated until all tracks contribute a $\chi^{2}$ value less than 4.0 or else there are fewer than three tracks remaining, in which case no secondary vertex is reconstructed for this particular jet. For jets with a reconstructed secondary vertex, the decay length projected onto the momentum vector of the jet in the $r$ - $\phi$ plane, $L$, is calculated with respect to the primary vertex, along with its error, $\sigma_{L}$. The primary vertex is reconstructed for each event using a $\chi^{2}$ minimization method which also incorporates the average beam spot position derived from the data as a constraint [14]. The average beam spot position itself is used for events failing the primary vertex finding, amounting to less than $0.1 \%$ of the total number of events. The sign of the decay length is obtained by summing the three-momenta of the tracks fitted to the secondary vertex; $L>0$ if the secondary vertex is displaced from the primary vertex in the same hemisphere as this momentum sum, and $L<0$ otherwise.

To identify quark jets, we require that one of the lower energy jets in the normal-mixture sample contains a reconstructed secondary vertex with decay length significance, defined by $L / \sigma_{L}$, greater than 5.0, and that the other lower energy jet does not contain a secondary vertex with $L / \sigma_{L}$ greater than 2.0. Furthermore, the decay length $L$ of the secondary vertex in the tagged jet is required to be less than $0.6 \mathrm{~cm} .{ }^{4}$ Additionally, the tracks fitted to the secondary vertex are examined. A track is deemed to be "significant" if its signed impact parameter value in the $r$ - $\phi$ plane with respect to the primary vertex, $b$, satisfies $b / \sigma_{b}>2.5$, with $\sigma_{b}$ the error of $b$. The sign of $b$ is assigned in the same way as described above for $L$. The event is retained

\footnotetext{
${ }^{4}$ The mean decay length of $\mathrm{b}$ hadrons at LEP, projected into the $r-\phi$ plane, is about $0.2 \mathrm{~cm}$.
} 
as a tagged event if the secondary vertex contains at least two significant tracks, in addition to the criteria given above.

Fig. 1(a) shows the distribution of $L / \sigma_{L}$ for the two lower energy jets of the normal-mixture sample added together, with jets defined using the $k_{\perp}$ algorithm, after all the requirements given above except for those concerning $L / \sigma_{L}$ have been imposed. The corresponding distribution for the cone algorithm is shown in Fig. 1(b). The distributions are peaked at $L / \sigma_{L}$ values near to 6.0 rather than near zero because two significant tracks are required to be present in the vertices. The tag interval $L / \sigma_{L}>5.0$ used to identify quark jets is indicated by the vertical dotted lines and arrows. The histogram shows the Monte Carlo prediction from the Jetset parton shower model [15] using the Peterson fragmentation function [16] for heavy quark production. Our implementation of this model is discussed in section 5.1. The Monte Carlo sample includes simulation of the OPAL detector [17] and the same selection criteria and analysis procedures as the data. The overall description of the data by the Monte Carlo is seen to be adequate. A slight shift to larger $L / \sigma_{L}$ values is observable in the data distributions compared to the Monte Carlo predictions. This small discrepancy is most likely related to somewhat small values for the mean $b$ hadron lifetime and the mean charged particle multiplicity in $b$ hadron decays used in the simulation. The effects of the uncertainty due to these $b$ hadron characteristics are included in our evaluation of the systematic uncertainties, as discussed in section 5.1.

Starting with the normal-mixture samples discussed in the previous section, 2416 tagged events are obtained using the $k_{\perp}$ jet finder and 2079 events using the cone one: we refer to these events as the "tagged" samples. About 55\% of the tagged events found using the cone algorithm are also found using the $k_{\perp}$ algorithm. The mean energy values of the jets in the tagged and normal-mixture samples are consistent with each other to within the statistical uncertainties, for both jet definitions. The tagged samples are subsets of the normal-mixture ones, but since they are small subsets we treat the tagged and normal-mixture data as though they are statistically independent of each other.

An example of an event tagged in this manner is shown in Fig. 2. Fig. 2(a) shows the full event view, projected into the $r$ - $\phi$ plane. The one-fold symmetric nature of the event with respect to the highest energy jet (upper right quadrant) is evident. Fig. 2(b) shows an enlarged view in the region of the silicon microvertex detector. An enlarged view in the vicinity of the $\mathrm{e}^{+} \mathrm{e}^{-}$collision point is shown in Fig. 2(c). In Fig. 2(c), a displaced secondary vertex is visible in the lower energy jet on the left side of the event, while all the tracks of the other lower energy jet (bottom side) converge towards the primary vertex. The jet on the left side is tagged as a quark jet while that on the bottom is identified as a gluon jet by anti-tagging. A displaced secondary vertex is also visible in the highest energy jet of the event, in the upper right quadrant of Fig. 2(c), consistent with a quark origin for that jet.

\section{Correction method}

To obtain fully corrected information for the quark and gluon jet data, we apply a two step procedure. First, the measured distributions are corrected for residual quark and gluon jet misidentification using an algebraic technique. Second, the data are multiplied bin-by-bin by correction factors to account for detector acceptance and resolution. We next describe these 
two correction steps. Following this, we describe a method to check the consistency of the Monte Carlo estimates of the jet purity values, using the data.

\subsection{Correction for quark and gluon jet misidentification}

The algebraic method used to correct for quark and gluon jet misidentification is the same as that which we introduced in [4]. If $D_{\text {n.mix }}\left(z_{i}\right)$ represents a distribution constructed from the two lower energy jets of the normal-mixture sample, with $z_{i}$ the content of bin $i$, then

$$
D_{\text {n.mix }}\left(z_{i}\right)=\left(1-q_{\text {n.mix }}\right) \cdot G\left(z_{i}\right)+q_{\text {n.mix }} \cdot Q\left(z_{i}\right),
$$

where $G\left(z_{i}\right)$ and $Q\left(z_{i}\right)$ are the distributions for pure gluon and pure quark jet states, respectively, with $q_{\text {n.mix }}$ the fraction of the jets which are quark jets. Analogously, if $D_{\text {g.tag }}\left(z_{i}\right)$ represents the distribution constructed from the anti-tagged gluon jets of the tagged sample, then

$$
D_{\text {g.tag }}\left(z_{i}\right)=\left(1-q_{\text {g.tag }}\right) \cdot G\left(z_{i}\right)+q_{\text {g.tag }} \cdot Q\left(z_{i}\right),
$$

where $q_{\mathrm{g} \text {.tag }}$ is the fraction of quark jets in the anti-tagged gluon jet sample. For perfect gluon jet identification, $q_{\mathrm{g} . t a g}$ would be zero. In reality, $q_{\mathrm{g} \text {.tag }}$ is not zero because of imperfect heavy quark jet identification using secondary vertices and because the highest energy jet in an event is sometimes the gluon jet. Assuming that $G\left(z_{i}\right)$ is the same in relations (2) and (3), and that the same holds true for $Q\left(z_{i}\right)$, the two equations may be inverted for $G\left(z_{i}\right)$ and $Q\left(z_{i}\right)$ once the coefficients $q_{\text {n.mix }}$ and $q_{\text {g.tag }}$ are known. The validity of these assumptions is discussed below. This correction method introduces bin-to-bin statistical fluctuations for $Q\left(z_{i}\right)$ which are about the same size and are anti-correlated with those of $G\left(z_{i}\right)$. Therefore, for distributions where it is appropriate, we present not only the measured gluon and quark jet distributions but also their ratio, in which correlations can easily be taken into account for the statistical uncertainties.

The values of $q_{\text {n.mix }}$ and $q_{\text {g.tag }}$ are estimated using events generated with the Jetset Monte Carlo program [15], version 7.3. Each simulated hadron jet is associated with an underlying quark or gluon jet. To perform this association, we employ the following method. Monte Carlo events which include simulation of the OPAL detector and the same event selection and analysis procedures as the data are examined at the parton level. The two hadron jets closest in angle to the directions of the primary quark and anti-quark which have evolved from the $\mathrm{Z}^{0}$ decay are considered to be the quark jets; the remaining jet is identified as the gluon jet. Jetset with its standard hadronization mechanism is known to yield too hard an energy spectrum for $b$ hadrons. Therefore we use Jetset in a non-default mode, in which $\mathrm{c}$ and $\mathrm{b}$ quark hadronization are described by the fragmentation function of Peterson et al. [16], in which case Jetset provides a much better description of the $b$ hadron energy spectrum measurements from LEP. The parameter values we use for Jetset result from a fit to OPAL measurements of global event shape distributions and are given in [4]. Using Jetset, the coefficients $q_{\text {n.mix }}$ and $q_{\text {g.tag }}$ are found to have the values $q_{\text {n.mix }}=0.516 \pm 0.001$ (stat.) \pm 0.007 (syst.) and $q_{\text {g.tag }}=0.070 \pm$ 0.005 (stat.) \pm 0.014 (syst.) for the $k_{\perp}$ jet finder, and $q_{\mathrm{n} \text {.mix }}=0.516 \pm 0.001$ (stat.) \pm 0.007 (syst.) and $q_{\text {g.tag }}=0.073 \pm 0.006$ (stat.) \pm 0.023 (syst.) for the cone jet finder. The gluon jet purity we obtain, given by $1-q_{\mathrm{g} \text {.tag }}$, is therefore about $93 \%$ for both jet finding methods. The same results are obtained using the Herwig parton shower model [18] (discussed in section 6), to within the statistical uncertainties. 
Three sources of systematic uncertainty for $q_{\mathrm{g} \text { tag }}$ are considered. First, the uncertainty due to imperfect knowledge of the $b$ hadron production and decay mechanism is evaluated by varying parameters in the Monte Carlo by the error ranges of current measurements, as follows.

1. The mean $b$ hadron lifetime was varied by $\pm 0.10 \mathrm{ps}[19]$ about a central value of $1.55 \mathrm{ps}$.

2. The Peterson parameter $\epsilon_{\mathrm{b}}$ that controls the $\mathrm{b}$ quark fragmentation function was varied from 0.0025 to 0.0095 , from a central value of 0.0057 , corresponding to a change in the mean scaled energy of $\mathrm{b}$ hadrons, $\left\langle x_{E}\right\rangle$, of from 0.68 to $0.72[20]$.

3. The mean charged particle multiplicity in $\mathrm{b}$ hadron decays including $\Lambda$ and $\mathrm{K}_{s}^{0}$ decay products, $\left\langle n_{\mathrm{ch} .}\right\rangle_{\mathrm{B}}$, was varied by \pm 0.5 tracks [21] about a central value of 5.2 .

4. The relative coupling strength of the $\mathrm{Z}^{0}$ to $\mathrm{b}$ quarks, $\Gamma_{\mathrm{b} \overline{\mathrm{b}}} / \Gamma_{\text {hadron }}$, was varied by $\pm 0.002[22]$ from a central value of 0.218 .

The systematic uncertainty associated with each item was taken to be the maximum variation in $q_{\text {g.tag }}$ that was observed relative to the standard result. The uncertainties in $q_{\mathrm{g} . t a g}$ related to the Peterson parameter $\epsilon_{\mathrm{c}}$ for the $\mathrm{c}$ quark fragmentation function and to the relative $\mathrm{Z}^{0}$ coupling strength to $\mathrm{c}$ quarks, $\Gamma_{\mathbf{c} \overline{\mathbf{c}}} / \Gamma_{\text {hadron, }}$, were evaluated in the same manner and were found to be negligible. Second, the uncertainty due to imperfect modeling of the detector is assessed by taking the maximum difference found relative to the standard result if the track impact parameter resolution in the detector simulation is improved or degraded by $20 \%$. Variation of the Monte Carlo resolution values for the other track parameters is found to cause no additional uncertainty. Third, the uncertainty due to the ambiguity of defining whether a jet originates from a quark or a gluon is evaluated by employing an alternative technique to make this association relative to the method presented above. Using Jetset events with detector simulation which have satisfied the same selection requirements as the data, three jets are reconstructed at the parton level and are matched to the hadron jets by finding the combination which minimizes the sum of the angular differences between them. ${ }^{5}$ The jets which contain the primary quark and anti-quark, after their evolution has terminated, are taken to be the quark jets, while the remaining jet is taken to be the gluon jet. For about $1.5 \%$ of the events defined using the $k_{\perp}$ jet finder, and $3.0 \%$ of the events defined using the cone jet finder, the primary quark and anti-quark are assigned to the same jet: these events are not used to estimate the jet purities for this alternative method to associate hadron and parton jets. The difference in purity values obtained using the two techniques to identify quark and gluon jets in the Monte Carlo is taken as the estimate of the systematic uncertainty associated with the jet definition ambiguity. The results of these systematic studies are summarized in Table 1. The total systematic uncertainties given above for $q_{\text {g.tag }}$ are estimated by adding the different contributions in quadrature. The systematic errors given above for $q_{\mathrm{n} \text {.mix }}$ are evaluated in an analogous manner.

Our method to correct for quark and gluon jet misidentification is based on the assumption that the gluon jet distribution $G\left(z_{i}\right)$ is the same in relations (2) and (3). This is the principal assumption of our analysis technique: that the gluon jets have the same properties in the

\footnotetext{
${ }^{5}$ For the $k_{\perp}$ jet finder, we require three jets to be reconstructed at the parton level; for the cone jet finder, the purity estimates for the alternative technique to associate hadron jets with quarks and gluons are based on the $86 \%$ of the events which are found to yield three jets at the parton level using the standard jet resolution choices.
} 
normal-mixture and tagged data sets. The justification for this assumption is that the gluon jets are selected under the same circumstances in both cases: they appear in the same kinematic configuration and are defined using the same track and jet finding criteria. There is a large difference between the quark flavor composition of the normal-mixture and tagged data sets, which we list in the top two rows of Table 2, but the properties of hard, acollinear gluon jets do not depend on the event flavor according to QCD [23]. Other possible biases in the gluon jet properties introduced by the analysis technique have been assessed using the Monte Carlo and are found to be essentially negligible. ${ }^{6}$ A second assumption of our analysis is that the quark jet distribution $Q\left(z_{i}\right)$ is the same in relations (2) and (3): thus that the quark jet background to the anti-tagged gluon jet data $\left(Q\left(z_{i}\right)\right.$ in relation $\left.(3)\right)$ has the same properties as the quark jets in the normal-mixture sample $\left(Q\left(z_{i}\right)\right.$ in relation (2)). This assumption is not entirely correct, because one of the principal sources of quark jet background in relation (3) is from events in which the gluon jet has the highest energy, recoiling against two $b$ quark jets in the opposite hemisphere. For these events, $Q\left(z_{i}\right)$ in relation (3) has an enhanced b quark component compared to $Q\left(z_{i}\right)$ in relation (2). The other source of background in relation (3) arises when the gluon jet is tagged, rather than the quark jet: these events occur mostly when two tracks from the decay of a $\Lambda$ or $\mathrm{K}_{s}^{0}$ hadron in the gluon jet are combined with a third track to define a secondary vertex. From Monte Carlo study, the overall bias due to these effects is found to be small compared to the statistical uncertainties. Residual effects are removed by the correction procedure for detector acceptance and resolution, described below, since the Monte Carlo is expected to simulate the relevant features with sufficient accuracy.

An explicit breakdown of the flavor composition of Monte Carlo events with correctly and incorrectly identified gluon jets in relation (3) is given in the last three rows of Table 2 for the analysis based on the $k_{\perp}$ jet definition. From the last row of this table, it is seen that events in which the gluon jet has the vertex tag exhibit a somewhat different flavor composition than the normal-mixture sample. This implies a small violation of our assumption that the gluon jet properties are independent of the quark flavor. Specifically, it is seen that there is an excess of about $10 \%$ in $\mathrm{s}, \mathrm{c}$ and $\mathrm{b}$ events compared to $\mathrm{d}$ and $\mathrm{u}$ events, which corresponds to about $1 \%$ of the events in the entire tagged sample. From further study, this excess is observed to be related to tracks near the cores of quark jets which are assigned to the gluon jet by the $k_{\perp}$ jet finder: $\mathrm{s}$, $\mathrm{c}$ and $\mathrm{b}$ jets contain a larger proportion of tracks from $\Lambda$ and $\mathrm{K}_{s}^{0}$ decays than $\mathrm{d}$ and $\mathrm{u}$ jets, explaining the excess of events with these three flavor types in this background class. For the cone jet finder, tracks near the quark jet cores are virtually never assigned to the gluon jet: the corresponding analysis reveals that events in which the gluon jet has the vertex tag do not exhibit a significant deviation from the flavor composition of the normal-mixture sample. The level of events with a potential bias in the gluon jet properties is therefore estimated to be well below $1 \%$ in this case. Residual effects related to this bias are removed by the detector corrections since they are included in the Monte Carlo simulations.

\subsection{Correction for detector acceptance and resolution}

A bin-by-bin multiplicative method is used to correct the measured gluon and quark jet distributions $G\left(z_{i}\right)$ and $Q\left(z_{i}\right)$ for the effects of detector acceptance and resolution. The correction

\footnotetext{
${ }^{6}$ There is a known bias of the gluon jet properties for only about $1 \%$ of the tagged events, using the $k_{\perp}$ jet finder, and for less than $1 \%$ of the tagged events, using the cone jet finder, as discussed below.
} 
factors are obtained using two different samples of Jetset Monte Carlo events. One sample includes initial-state photon radiation, simulation of the OPAL detector and the same selection and analysis procedures as the data, including the secondary vertex reconstruction and algebraic procedure described in section 5.1 to obtain pure quark and gluon jet information. Good agreement is found between the gluon and quark jet distributions derived from this Monte Carlo sample and the $G\left(z_{i}\right)$ and $Q\left(z_{i}\right)$ data distributions, respectively, for all variables studied. The second Monte Carlo sample does not include initial-state photon radiation or detector simulation and treats all charged and neutral particles with mean lifetimes greater than $3 \cdot 10^{-10} \mathrm{~s}$ as stable. The same three-jet event selection criteria as described in section 3 are applied except that the jets are not required to satisfy $|\cos \theta|<0.9$. The quark and gluon jets in this second sample are identified with Monte Carlo information using the method given in section 5.1. The multiplicative correction factors are obtained by taking the ratios of the distributions predicted by the second sample to those predicted by the first one on a bin-by-bin basis. The distributions $Q\left(z_{i}\right)$ and $G\left(z_{i}\right)$ are multiplied bin-by-bin by these correction factors to obtain the fully corrected quark and gluon jet measurements. The good agreement between the distributions constructed using the first Monte Carlo sample described above and $G\left(z_{i}\right)$ and $Q\left(z_{i}\right)$ justifies this bin-by-bin technique. The values of the detector correction factors are presented along with the data in sections 6 and $7:$ their values mostly lie between 0.8 and 1.2 .

\subsection{Consistency check of the jet purity values}

There is an almost linear relationship between the purity of the gluon jets and the $b$ quark fraction in the Monte Carlo event samples since we mainly tag jets using b quark identification. A consistency check of the gluon jet purity values derived from the Monte Carlo may therefore be performed by testing the Monte Carlo predictions for the $b$ quark fractions. One measure of the $b$ quark fraction is the rate at which secondary vertices are reconstructed in the highest energy jets of the events: these jets are almost $100 \%$ quark jets and have not been biased by any tagging conditions in our analysis.

The open points in Fig. 3(a) show the Monte Carlo results obtained by changing the quark tagging conditions in the lower energy jets of the normal-mixture sample and examining the fraction of the tagged events for which a secondary vertex with $L / \sigma_{L}>5$ is also reconstructed

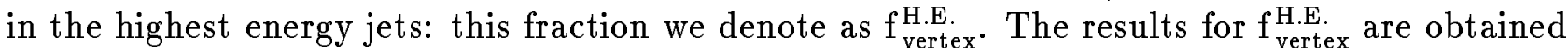
using the $k_{\perp}$ jet finder and are plotted versus the gluon jet purity $1-q_{\text {g.tag }}$ in the events. The right-most point corresponds to the standard tagged sample, with $1-q_{\mathrm{g} \text {.tag }}=0.93$. The left-most point corresponds to the normal-mixture sample (no tagging of the lower energy jets), with $1-q_{\text {n.mix }} \approx 0.48$. The intermediate points are found by applying less restrictive tagging criteria for the lower energy jets than we employ for the standard analysis. This plot establishes the linear relationship between the gluon jet purity and the $b$ quark fraction. The solid points

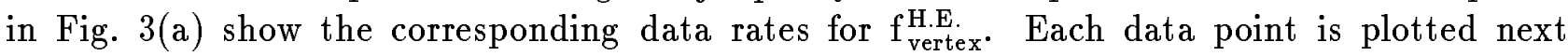
to the Monte Carlo one having the same tagging condition for the lower energy jets. Note that only the ordinate in Fig. 3(a) may be measured from the data. However, the abscissa value of the left-most point, corresponding to the normal-mixture sample, is determined as a simple consequence of the gluon radiation spectrum: if the highest energy jet is a quark jet for $f_{q} \approx 97 \%$ of the events, then one of the lower energy jets must be a gluon jet in $f_{q} / 2 \approx 48 \%$ of the events. Thus the gluon jet purity value given for the normal-mixture data point does 
not have a large uncertainty for the purposes of this analysis. Also, the $b$ quark fraction for the normal-mixture data point comes essentially from $\Gamma_{\mathrm{b} \overline{\mathrm{b}}} / \Gamma_{\text {hadron }}$ and so also does not have an important uncertainty for our purposes. The analogous plot to Fig. 3(a) for the cone jet finder yields values for $\mathrm{f}_{\text {vertex }}^{\mathrm{H} . \mathrm{f} \text {. }}$ which are about $5 \%$ larger in the data relative to the Monte Carlo irrespective of the tagging condition in the lower energy jets. Since the discrepancy is also present for the normal-mixture sample for which the gluon jet purity and $b$ quark fraction are believed to be understood, this discrepancy is likely to be due to a difference between the Monte Carlo and data efficiencies for reconstructing secondary vertices using this jet finder rather than to a difference between the gluon jet purity values.

Therefore, to test the Monte Carlo using a measurable quantity related to the gluon jet purity, we show in Figs. 3(b) and (c) the ratio of the observed rates at which secondary vertices are observed in the highest energy jets of the tagged and normal-mixture events, displayed in

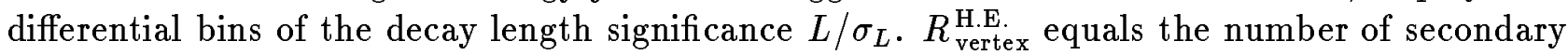
vertices in a certain bin of $L / \sigma_{L}$ for the highest energy jets, normalized by the number of events in the sample. The results found using the standard criteria for the tagged samples (yielding 93\% estimated gluon jet purity) are shown by the solid points. The results found using an alternative lifetime tagging algorithm to identify quark jets (yielding about $78 \%$ estimated gluon jet purity) are shown by the open triangles. This alternative method to identify quark jets is used as a systematic check in our analysis and is described in section 8. Fig. 3(b) shows the results obtained using the $k_{\perp}$ jet finder. Those found using the cone jet finder are shown in Fig. 3(c). The right-most bin in Figs. 3(b) and (c) includes all $L / \sigma_{L}$ values larger than 30 .

For values of $L / \sigma_{L}$ below about 5.0, the distributions shown in Figs. 3(b) and (c) are dominated by light quark jets and the observed rates do not differ much between the normalmixture and tagged data sets: thus the values of the ratios are approximately unity. For values of $L / \sigma_{L}$ above about 5.0, the secondary vertex rate in the highest energy jets is seen to be three to four times larger in the tagged samples than it is in the normal-mixture ones, using the standard tagging criteria, because of the enhanced b quark component of the tagged data sets. For the alternative tagging algorithm, the ratio values for $L / \sigma_{L}>5$ are lower since the jet purities achieved using this method are not as high. The corresponding results from the Monte Carlo are shown by the solid and dashed histograms in Figs. 3(b) and (c). The Monte Carlo agrees well with the data for both jet finders and for both quark tagging algorithms, demonstrating that the enhancement factor for $b$ quarks in the tagged samples, relative to the known $b$ quark fraction in the normal-mixture samples, is well simulated. This makes it plausible that the Monte Carlo estimates for $q_{\mathrm{g} \text {.tag }}$ are reliable.

\section{$6 \quad$ Results using the $k_{\perp}$ jet finder}

In this section, we present our results for quark and gluon jet properties measured using the $k_{\perp}$ jet definition. Systematic uncertainties for these data are discussed in section 8 . The data are compared to the predictions of the Jetset, Herwig [18], Ariadne [24] and Cojets [25] parton shower models. Our implementation of Jetset was discussed in section 5.1. The version of Herwig we use is 5.6; that we use for Ariadne is 4.06. The parameter values of these two models were tuned by us to provide a good description of global event characteristics in $\mathrm{Z}^{0}$ decays and are given in [4] and [26], respectively. For Cojets, we examine the predictions of 
two different versions, 6.12 and 6.23, and use the default parameter sets provided by the Monte Carlo author, which also yield good descriptions of the global event characteristics in hadronic $\mathrm{Z}^{0}$ decays. For version 6.12 , there is essentially no difference between quark and gluon jet properties. This version therefore provides a useful "toy model" with which to demonstrate the non-triviality of our results for quark and gluon jet differences. For version 6.23 , differences between quark and gluon jets have been implemented. The Monte Carlo samples are generated in the same manner as the Jetset sample without detector simulation discussed in section 5.2.

\subsection{Jet width distributions}

One of the characteristics which is expected to differ between quark and gluon jets is the angular distribution of jet energy with respect to the jet axis. Fig. 4 shows the normalized distribution of the quark and gluon jet energy around the jet axis, $\left(1 / E^{\text {jet }}\right)\left(\mathrm{d} E^{\text {jet }} / \mathrm{d} \chi\right) \mathrm{d} \chi$ versus $\chi$, where $\chi$ is the angle between a particle and the axis of the jet to which it is assigned. This distribution is constructed by calculating the fraction of the jet visible energy contained in annular rings around the axis, on an event-by-event basis, and then accumulating the information from each event to obtain the histogram shown. The distributions shown in Fig. 4 extend out to $60^{\circ}$ from the jet axis and encompass more than $99 \%$ of the jet visible energy for both the quark and gluon jets. The bin-by-bin factors used to correct for detector acceptance and resolution are shown in the small figure above the data distributions. A minimum bin size of $2^{\circ}$ is chosen to match the estimated resolution with which the jet direction is determined. This estimate is obtained by comparing the jet directions in the Monte Carlo before and after the effects of the detector and track selection have been included: an RMS difference of $2.3^{\circ}$ is found for the two lower energy jets.

Comparing the quark jet data, shown by the solid points, to the gluon jet data, shown by the open points, it is seen that the gluon jet energy is markedly less collimated around the jet direction than is the quark jet energy. Thus gluon jets are observed to be broader than quark jets, in agreement with our earlier findings [3, 4]. This confirms one of the general expectations for quark and gluon jet differences predicted by QCD, mentioned in the introduction. The ratio of the gluon to the quark jet measurements is presented in Fig. 5. The correlations introduced by the algebraic procedure to obtain pure quark and gluon jet information are taken into account in the errors shown. (This comment is true for all the ratio values between gluon and quark jet data presented in this paper.) Numerical values for the data in Figs. 4 and 5 are given in Table 3.

Fig. 4 also shows the predictions of the Jetset, Herwig and Ariadne models. The predictions of Cojets are not included in Fig. 4 in order to keep the presentation of the data relatively simple. All three models are seen to provide an adequate description of the measurements. In Fig. 5, the predictions of the models, including those of Cojets, are shown. Cojets 6.23 is seen also to describe the data adequately, while Cojets 6.12 , which does not include a significant quark and gluon jet difference as noted above, is in disagreement with the data, as expected. 


\subsection{Charged particle fragmentation function}

A second feature which is expected to differ between quark and gluon jets is the inclusive distribution of particle energy in the jets, known as the fragmentation function. Fig. 6 shows the charged particle fragmentation function, $\left(1 / N_{\text {event }}\right) \mathrm{d} n_{\text {ch. }} / \mathrm{d} x_{E}$ versus $x_{E}$, of the quark and gluon jets, where $x_{E}=E / E^{\text {jet }}$ is the scaled energy of a particle with respect to the energy of the jet to which it is assigned. Note that there are no data in the last bin of the gluon jet fragmentation function, corresponding to $0.80<x_{E}<1.0$. The distributions are normalized so that the integral, $\int_{0}^{1}\left[\left(1 / N_{\text {event }}\right) \mathrm{d} n_{\text {ch. }} / \mathrm{d} x_{E}\right] \mathrm{d} x_{E}$, gives the mean charged particle multiplicity of the quark or gluon jets. This differs from the normalization used in our earlier publications [3, 4], in which the corresponding integrals were unity. In all cases, the bin size is considerably larger than the corresponding energy resolution estimated for particles in the bin. The ratio of the gluon to the quark jet distributions is shown in Fig. 7. Numerical values for these measurements are given in Table 4.

The detector corrections for the first bin of Fig. 6, corresponding to $x_{E}<0.01(E<0.25 \mathrm{GeV})$, are large since charged particles with $p_{\perp}$ values below $0.15 \mathrm{GeV} / c$ are not included in the analysis, as mentioned in section 2. The detector corrections for this bin have values of 5.8 and 3.5 for the gluon and quark jets, respectively. ${ }^{7}$ The corresponding correction for the first bin of the ratio distribution, Fig. 7, is 1.6 .

From Fig. 6, it is seen that gluon jets contain substantially fewer particles with large energies than quark jets, in agreement with our earlier results $[3,4]$ and the general expectations of QCD mentioned in the introduction. From Fig. 6, Jetset and Ariadne are seen to provide a reasonable description of the measurements. The Herwig predictions at large $x_{E}$ values are somewhat below the data for both the quark and gluon jet distributions. The Cojets predictions, shown in Fig. 7, demonstrate that version 6.23 describes the overall trend of the data, although it does not agree with them as well as Jetset, Herwig or Ariadne, while version 6.12 is in strong disagreement with the experimental observations, again as expected.

\subsection{Mean charged multiplicity}

A third property which is expected to differ between quark and gluon jets is the mean particle multiplicity. The mean charged particle multiplicities of the gluon and quark jets are measured to be $\left\langle n_{k_{\perp}}^{\text {ch. }}\right\rangle_{\text {gluon }}=9.10 \pm 0.07$ (stat.) \pm 0.09 (syst.) and $\left\langle n_{k_{\perp}}^{\text {ch. }}\right\rangle_{\text {quark }}=7.27 \pm 0.07$ (stat.) \pm 0.08 (syst.), where the systematic uncertainties are discussed in section 8 . These values have been corrected using the procedures of section 5 , treating the mean multiplicity as a distribution with a single bin. We obtain the same results to within very small differences if we integrate the fragmentation functions to obtain the mean multiplicity values. The ratio, $\left\langle n_{k_{\perp}}^{\text {ch. }}\right\rangle_{\text {gluon }} /\left\langle n_{k_{\perp}}^{\text {ch. }}\right\rangle_{\text {quark }}$, is $1.251 \pm 0.024$ (stat.) \pm 0.029 (syst.). Our results for the mean charged particle multiplicity values are summarized in the top row of Table 5 , in which the correction factors used to account for detector effects are also given. A comparison of these results to the predictions of the QCD models is presented in section 6.4 .

\footnotetext{
${ }^{7}$ Repeating the analysis with the same event samples but using charged tracks with momentum $p$ larger than $0.10 \mathrm{GeV} / c$ yields detector corrections of 1.1 and 1.5 for the quark and gluon jets in this bin; the change in the corrected data values is negligible.
} 
Thus, using the $k_{\perp}$ jet finder, the gluon jets are observed to yield a mean charged particle multiplicity value which is about $25 \%$ larger than that of the quark jets, in agreement with our earlier result [4] based on the same jet definition. ${ }^{8}$ Our result also confirms the general expectation of QCD that the mean particle multiplicity of gluon jets should exceed that of quark jets, mentioned in the introduction. (See [27] for a discussion of how these experimental results should be interpreted in comparison to theory [1].) Recently, the ALEPH Collaboration [28], using a similar method to ours [4], has obtained a similar value for $\left\langle n_{k_{\perp}}^{\text {ch. }}\right\rangle_{\text {gluon }} /\left\langle n_{k_{\perp}}^{\text {ch. }}\right\rangle_{\text {quark }}$.

\subsection{Sensitivity to $\boldsymbol{y}_{\text {cut }}$}

To investigate the sensitivity of the results presented above to the choice of the jet resolution parameter, $y_{c u t}$, we repeated the analysis using $y_{\text {cut }}$ values of 0.005 and 0.05 . These values of $y_{\text {cut }}$ were chosen because they differ widely from the value of 0.02 chosen for our standard analysis, while they are not so large or so small that the event statistics are reduced by too large a factor. With $y_{c u t}=0.005,1681$ events are selected in the tagged sample, compared to 1411 events using $y_{c u t}=0.05$. About $94 \%$ of the events selected using $y_{\text {cut }}=0.005$ are also selected in the standard analysis using $y_{\text {cut }}=0.02$; the corresponding number for $y_{\text {cut }}=0.05$ is also $94 \%$. Of the events selected using $y_{c u t}=0.005$, only $47 \%$ are also selected using $y_{c u t}=0.05$, however.

The smaller $y_{\text {cut }}$ value yields somewhat narrower jets compared to those observed using $y_{c u t}=0.02$. To illustrate this, Fig. 8(a) shows the fractional difference between the results found for $y_{\text {cut }}=0.005$ and $y_{\text {cut }}=0.02$, for the $\left(1 / E^{\text {jet }}\right)\left(\mathrm{d} E^{\text {jet }} / \mathrm{d} \chi\right) \mathrm{d} \chi$ versus $\chi$ distribution (Fig. 4). The larger $y_{c u t}$ value yields slightly broader jets compared to the standard analysis, as illustrated in Fig. 8(b), which shows the fractional difference between the results found using $y_{\text {cut }}=0.05$ and $y_{c u t}=0.02$. The differences between the results obtained for the different $y_{c u t}$ values are observed to be generally modest compared to the differences between the quark and gluon jet curves in Fig. 4. The ratio of the gluon to quark jet data, shown in Fig. 5, is virtually unaffected by the $y_{\text {cut }}$ choice since the numerator and denominator behave in essentially the same manner as $y_{\text {cut }}$ changes, as seen from Fig. 8. Thus the $y_{\text {cut }}$ choice does not influence the quark-gluon jet width differences we observe in a significant manner.

A similar study for the charged particle fragmentation functions of Fig. 6 reveals that $y_{c u t}=0.05$ yields somewhat softer spectra for both the quark and gluon jet data compared to $y_{c u t}=0.005$, but that, again, the difference is modest compared to the difference between the quark and gluon jet results. The ratio of the gluon to quark jet measurements shown in Fig. 7 is largely unaffected by the $y_{\text {cut }}$ choice, although there is a slight tendency for the differences between quark and gluon jets to increase with $y_{c u t}$.

The sensitivity of the mean charged particle multiplicity values to $y_{\text {cut }}$ is shown in Fig. 9(a): $\left\langle n_{k_{\perp}}^{\text {ch. }}\right\rangle_{\text {gluon }}$ and $\left\langle n_{k_{\perp}}^{\text {ch. }}\right\rangle_{\text {quark }}$ decrease by $3-4 \%$ for $y_{\text {cut }}=0.005$, and increase by about the same amount for $y_{c u t}=0.05$, compared to the standard results. The ratio of the gluon to quark jet measurements, $\left\langle n_{k_{\perp}}^{\text {ch. }}\right\rangle_{\text {gluon }} /\left\langle n_{k_{\perp}}^{\text {ch. }}\right\rangle_{\text {quark }}$, exhibits almost no dependence on $y_{\text {cut }}$, however, as shown in Fig. 9(b). Fig. 9 demonstrates again that Jetset, Herwig and Ariadne are all generally

\footnotetext{
${ }^{8}$ Our earlier result, $\left\langle n_{k_{\perp}}^{\text {ch. }}\right\rangle_{\text {gluon }} /\left\langle n_{k_{\perp}}^{\text {ch. }}\right\rangle_{\text {quark }}=1.33 \pm 0.09$ (stat. + syst.), was not corrected for detector effects; the corresponding detector correction factor is found to be 1.013 and so is near unity.
} 
successful at describing the data, that Cojets version 6.23 is somewhat less successful, and that Cojets version 6.12 is in disagreement with them. The mean multiplicity measurements obtained using the alternative $y_{\text {cut }}$ choices are summarized in Table 5.

\section{$7 \quad$ Results using the cone jet finder}

Next, we present our results for quark and gluon jet properties measured using the cone jet definition. The comments given at the beginning of section 6 also apply here.

\subsection{Jet width distributions}

One of the principal motivations for introducing a cone jet definition in our analysis is to facilitate the comparison of jet properties between $\mathrm{e}^{+} \mathrm{e}^{-}$and $\mathrm{p} \overline{\mathrm{p}}$ experiments. To characterize the width of a jet, we therefore select variables which have been used at $p \bar{p}$ colliders. One such variable is the energy profile of a jet, $\psi_{\mathrm{E}}(r / R)[29]$, defined for a jet of half angle $R$ as the fraction of the jet energy contained within a smaller cone of half angle $r$ which is coaxial with the jet. A related variable is the differential energy profile, $\phi_{\mathrm{E}}(r / R)[29]$, defined by:

$$
\phi_{\mathrm{E}}(r / R) \equiv \frac{\mathrm{d} \psi_{\mathrm{E}}(r / R)}{\mathrm{d}(r / R)}
$$

with $\mathrm{d}(r / R)$ the bin width. To compare quark and gluon jets, $\phi_{\mathrm{E}}(r / R)$ is preferable to $\psi_{\mathrm{E}}(r / R)$ because the bin-to-bin contents are less correlated. The $\phi_{\mathrm{E}}(r / R)$ distribution is very similar to the $\left(1 / E^{\text {jet }}\right)\left(\mathrm{d} E^{\text {jet }} / \mathrm{d} \chi\right) \mathrm{d} \chi$ distribution studied in the case of the $k_{\perp}$ jet finder (Fig. 4$)$. In Fig. 10, we present our measurement of $\phi_{\mathrm{E}}(r / R)$ versus $r / R$ for the quark and gluon jets. A bin size of $\mathrm{d}(r / R)=(1 / 15)$ has been chosen because this matches the estimated angular resolution of about $2^{\circ}$ for determining the jet direction. Gluon jets are seen to be substantially broader than quark jets, in agreement with our results from the $k_{\perp}$ analysis. The differential charged particle multiplicity profile, $\phi_{\mathrm{M}}^{\text {ch. }}(r / R)$, defined in analogy to $\phi_{\mathrm{E}}(r / R)$, is shown in Fig. 11: the greater breadth of gluon jets compared to quark jets is also visible in this distribution. Figs. 12 and 13 display the corresponding integral profile distributions, $\psi_{\mathrm{E}}(r / R)$ and $\psi_{\mathrm{M}}^{\text {ch. }}(r / R)$, versus $r / R$, while the ratios of the gluon to quark jet measurements for $\phi_{\mathrm{E}}(r / R)$ and $\phi_{\mathrm{M}}^{\text {ch. }}(r / R)$ are presented in Figs. 14 and 15. Numerical values for these data are given in Tables 6 and 8 for the energy measurements and in Tables 7 and 9 for the multiplicity measurements.

Included in Figs. 10-13 are the predictions of the Jetset, Herwig and Ariadne models. In addition to these three models, the Cojets predictions are shown in Figs. 14 and 15.

In a previous publication [7], we studied the $\phi_{\mathrm{E}}(r / R)$ and $\psi_{\mathrm{E}}(r / R)$ distributions in our data using jets with energies of about $45 \mathrm{GeV}$ : these jets are almost always quark jets since they are close to the kinematic limit in $\mathrm{Z}^{0}$ decays. The $\mathrm{e}^{+} \mathrm{e}^{-}$distributions were compared to measurements of $\phi_{\mathrm{E}}(r / R)$ and $\psi_{\mathrm{E}}(r / R)$ for jets measured by the CDF Collaboration in $\mathrm{p} \overline{\mathrm{p}}$ collisions at a c.m. energy of $1.8 \mathrm{TeV}[29]$. The $\mathrm{p} \overline{\mathrm{p}}$ jets were produced in the central region of pseudorapidity, $0.1<|\eta|<0.7$, where $\eta=-\ln \left(\tan \frac{\theta}{2}\right)$, and also had energies of about $45 \mathrm{GeV}: \mathrm{p} \overline{\mathrm{p}}$ jets selected under these conditions are expected to be predominantly gluon jets. We observed 
that the jets from $\mathrm{p} \overline{\mathrm{p}}$ collisions were substantially broader than the ones from our experiment. The OPAL and CDF measurements of $\phi_{\mathrm{E}}(r / R)$ for the $45 \mathrm{GeV}$ jets are displayed in Fig. 16(a). A cone size of $R=57^{\circ}$ has been used. Shown in contrast to these measurements are the results from the present study for $24 \mathrm{GeV}$ quark and gluon jets with $R=30^{\circ}$. The CDF data have been corrected for the presence of underlying events using an energy density of $0.7 \mathrm{GeV} / R^{2}$ as explained in [7]. The uncertainties shown for the CDF data include the difference between the $0.7 \mathrm{GeV} / R^{2}$ corrected data and the data with no correction for underlying events.

Interpreting the results for the $45 \mathrm{GeV}$ jets to correspond to quark jets for OPAL and to gluon jets for $\mathrm{CDF}$, it is seen that the $45 \mathrm{GeV}$ jets are narrower than the $24 \mathrm{GeV}$ ones for both jet types. The difference between the $45 \mathrm{GeV}$ jets from the $\mathrm{e}^{+} \mathrm{e}^{-}$and $\mathrm{p} \overline{\mathrm{p}}$ experiments bears a striking resemblance to the difference between quark and gluon jets observed in the present study, however. This is emphasized in Fig. 16(b), in which the ratios of the $45 \mathrm{GeV}$ (solid points) and $24 \mathrm{GeV}$ (open points) gluon to quark jet distributions are displayed. A larger fractional difference is observed between the jet data at $45 \mathrm{GeV}$ than at $24 \mathrm{GeV}$. Monte Carlo study suggests that some of the difference between the 45 and $24 \mathrm{GeV}$ distributions in Fig. 16(b) is due to the different choice of cone size $R$, as is demonstrated by the dashed and dash-dotted curves in that figure which show the results we obtain from Jetset for the analysis described in this paper using cone sizes of $30^{\circ}$ and $50^{\circ}$, respectively, with $\epsilon=10 \mathrm{GeV}$. We also show the results yielded by Jetset for $45 \mathrm{GeV}$ jets with $R=50^{\circ}$, obtained by running the event generator for the analysis described in this paper at a $\mathrm{e}^{+} \mathrm{e}^{-}$c.m. energy of $175 \mathrm{GeV}$ rather than $91.2 \mathrm{GeV}$. Thus the results of Fig. 16 support the conclusion we made in [7]: that much of the difference between the $\mathrm{e}^{+} \mathrm{e}^{-}$and $\mathrm{p} \overline{\mathrm{p}}$ data can be attributed to differences between quark and gluon jet properties. We note that the metric used here to define the cone size $R$ differs from that used for the $\mathrm{p} \overline{\mathrm{p}}$ data. For the $\mathrm{p} \overline{\mathrm{p}}$ results, the jets are defined by $\sqrt{\Delta \eta^{2}+\Delta \phi^{2}}<R$, where $\Delta \eta$ and $\Delta \phi$ are the differences in pseudorapidity and azimuthal angle between the cone axis and a particle direction, with $\phi$ measured in radians. The difference between the $\eta$ - $\phi$ metric and the $\chi$ metric employed here was shown in [7] to be small for the central pseudorapidity region used for the $\mathrm{p} \overline{\mathrm{p}}$ analysis, however.

\subsection{Charged particle fragmentation function}

The charged particle fragmentation functions of the quark and gluon jets, $\left(1 / N_{\text {event }}\right) \mathrm{d} n_{\text {ch. }} / \mathrm{d} x_{E}$ versus $x_{E}$, are shown in Fig. 17. The ratio of the gluon to the quark jet data is presented in Fig. 18. It is seen that gluon jets have a strikingly softer particle energy spectrum than quark jets, in agreement with the results based on the $k_{\perp}$ jet definition. Numerical values for these measurements are given in Table 10. As for the case of the $k_{\perp}$ analysis, the detector corrections for the first bin of these distributions are large. The detector corrections for the first bin of Fig. 17 are 5.8 for the gluon jet and 3.5 for the quark jet, while that for the first bin of Fig. 18 is 1.7. The Monte Carlo predictions, included in Figs. 17 and 18, are seen to demonstrate the same trends relative to the data as were observed for the $k_{\perp}$ study (compare Figs. 17 and 18 to Figs. 6 and 7). 


\subsection{Mean charged multiplicity}

For the cone jet finder, the measured mean charged particle multiplicity values of the gluon and quark jets are found to be $\left\langle n_{\text {cone }}^{\text {ch. }}\right\rangle_{\text {gluon }}=6.26 \pm 0.06$ (stat. $) \pm 0.07$ (syst.) and $\left\langle n_{\text {cone }}^{\text {ch. }}\right\rangle_{\text {quark }}=5.71 \pm$ 0.05 (stat.) \pm 0.05 (syst.). These values are lower than those found using the $k_{\perp}$ jet finder since not all particles in an event are necessarily assigned to a jet. The ratio, $\left\langle n_{\text {cone }}^{\text {ch. }}\right\rangle_{\text {gluon }} /\left\langle n_{\text {cone }}^{\text {ch. }}\right\rangle_{\text {quark }}$, is $1.096 \pm 0.023$ (stat.) \pm 0.23 (syst.). These results, along with the values of the detector correction factors, are summarized in the top row of Table 11.

\subsection{Sensitivity to $R$ and $\epsilon$}

To investigate the sensitivity of our results to the choice of the cone size $R$, the analysis was repeated using $R$ values of $40^{\circ}$ and $50^{\circ}$, rather than $30^{\circ}$ as in the standard analysis, with $\epsilon$ fixed at $10 \mathrm{GeV}$ : this yielded 1616 and 485 tagged events, respectively. The analysis using $R=40^{\circ}$ shares $68 \%$ of its events with that using $R=30^{\circ}$; the corresponding number for $R=50^{\circ}$ is $47 \%$. To investigate the sensitivity of the results to the choice of the minimum visible jet energy $\epsilon$, the analysis was repeated using $\epsilon$ values of $5 \mathrm{GeV}$ and $15 \mathrm{GeV}$, with $R$ fixed at $30^{\circ}: 1844$ and 1245 tagged events were selected using these two alternative $\epsilon$ values, respectively. Of these events, $91 \%$ and $97 \%$ were also contained in the sample selected using $\epsilon=10 \mathrm{GeV}$. Of the events selected using $\epsilon=5 \mathrm{GeV}, 59 \%$ are contained in the sample selected using $\epsilon=15 \mathrm{GeV}$.

Fig. 19(a) shows the fractional difference between the results obtained using $R=30^{\circ}$ and $R=50^{\circ}$ for the differential jet energy profile, $\phi_{\mathrm{E}}(r / R)$ versus $r / R$ (Fig. 10). The quark jet data, shown by the solid points, demonstrate that the quark jets defined using $R=50^{\circ}$ have a larger fraction of their energy concentrated at small $r / R$ values than do the quark jets defined using $R=30^{\circ}$. The gluon jet data, shown by the open points, exhibit an opposite trend for small $r / R$ values. Thus the differences between the quark and gluon jet distributions become more pronounced as $R$ increases. Fig. 19(b) shows the fractional difference between the $\phi_{\mathrm{E}}(r / R)$ distributions obtained using $\epsilon=5 \mathrm{GeV}$ and $\epsilon=15 \mathrm{GeV}$. In this case, little difference is observed between the results found for the two different jet resolution choices. The features seen in Fig. 19 for the differential energy profile are also observed for the differential charged particle multiplicity profile, $\phi_{\mathrm{M}}^{\text {ch. }}(r / R)$ versus $r / R$ (Fig. 11), as the jet resolution parameters are varied.

For the charged particle fragmentation function, $\left(1 / N_{\text {event }}\right) \mathrm{d} n_{\mathrm{ch}} / \mathrm{d} x_{E}$, important differences are observed as $R$ changes because more soft particles are incorporated into the jets as the cone size increases. The gluon jet fragmentation functions found using $R=30^{\circ}$ and $R=50^{\circ}$ are shown in Fig. 20. For purposes of comparison, the corresponding distribution obtained using the $k_{\perp}$ jet definition with $y_{c u t}=0.02$ (Fig. 6) is included in Fig. 20. Fig. 21 shows an analogous comparison for the quark jet fragmentation functions. The spectra defined using $R=50^{\circ}$ (open points) are seen to be substantially softer than those defined using $R=30^{\circ}$ (solid points), as is especially pronounced for the gluon jet. In contrast to the differences observed when $R$ is varied, essentially no change is observed in the quark and gluon jet fragmentation functions if $\epsilon$ is varied between $5 \mathrm{GeV}$ and $15 \mathrm{GeV}$, however. Comparing the results for the $k_{\perp}$ jet finder (histogram) to the solid and open points in Fig. 20, it is seen that the $k_{\perp}$ result is softer than the cone one for $R=30^{\circ}$ and harder than the cone one for $R=50^{\circ}$. Smaller differences are observed between the results of the $k_{\perp}$ and cone definitions for the quark jet measurements 
shown in Fig. 21. For all jet definitions, the fragmentation function of the gluon jet is much softer than that of the quark jet.

The sensitivity of the mean charged particle multiplicity values to the cone size is shown in Fig. 22(a). For $R=50^{\circ}$, the mean multiplicity values of the gluon and quark jets are $40 \%$ and $22 \%$ larger than for $R=30^{\circ}$. The corresponding results for the ratio of the gluon to quark jet data are shown in Fig. 22(b): an increase is seen in this ratio as $R$ becomes larger. For $R=50^{\circ}$, $\left\langle n_{\text {cone }}^{\text {ch. }}\right\rangle_{\text {gluon }} /\left\langle n_{\text {cone }}^{\text {ch. }}\right\rangle_{\text {quark }}$ is found to have a value of 1.26 , compared to its value of 1.10 found for $R=30^{\circ}$ (section 7.3). In contrast to the variation which is observed as $R$ changes, little variation is observed in the multiplicity values as $\epsilon$ changes. Our results for the mean charged particle multiplicity values of quark and gluon jets obtained using the different jet resolution conditions are summarized in Table 11.

\section{Systematic checks}

To assess potential systematic uncertainties for the measurements presented above, we consider effects related to the measurement process and accuracy of the detector simulation program, the correction procedure, the track selection, and the three-jet event definition. Our evaluation of these uncertainties is discussed in the following paragraphs. First, we present the investigations which were performed, then the manner in which this information is used to evaluate systematic error. Following this, we present an additional systematic test based on a different lifetime tagging algorithm to identify quark jets. A last source of systematic uncertainty is due to the limited size of the Monte Carlo sample used to obtain the detector correction factors. This source contributes an uncertainty which is about $65 \%$ as large as the experimental statistical error.

The experimental systematic uncertainty, due to imperfections in the measurement process and simulation of the detector, was evaluated by repeating the analysis using charged tracks alone for both the data and detector-simulated Monte Carlo samples. The data were corrected back to the same level, including charged and neutral particles with mean lifetimes greater than $3 \cdot 10^{-10} \mathrm{~s}$, as in the standard analysis. The same selection criteria given in sections 2,3 and 4 were used. For the cone jet finder, an additional check was made for the distributions based on energy measurements (the $\psi_{\mathrm{E}}(r / R)$ and $\phi_{\mathrm{E}}(r / R)$ distributions) by repeating the analysis using only electromagnetic calorimeter clusters. For the analysis using the calorimeter only, all clusters - both those associated and those not associated with charged tracks - were used, and a requirement that at least eight clusters be present in an event replaced the requirement of at least five charged tracks. Otherwise the selection criteria were the same as for the standard analysis. Secondary vertices were defined in the calorimeter-only case by using charged tracks which fell within the cones defined by the clusters. A calorimeter-only analysis was not performed using the $k_{\perp}$ jet finder because it is not obvious how to assign charged tracks to the calorimeter-only jets, in order to identify secondary vertices, in a manner that preserves the integrity of the jet definition.

Two potential sources of systematic uncertainty associated with the correction procedure were investigated: the values of the algebraic correction coefficients $q_{\text {n.mix }}$ and $q_{\text {g.tag }}$ (relations (2) and (3)), and possible model dependence of the bin-by-bin factors used to account for 
detector effects. The first of these uncertainties was evaluated by repeating the analysis using values of $q_{\text {n.mix }}$ and $q_{\mathrm{g} \text {.tag }}$ which differed from their standard ones by their total uncertainties given in section 5.1. The second of these uncertainties was evaluated by using Herwig for the detector corrections instead of Jetset.

Potential sensitivity of the results to details of the track selection was assessed by repeating the analysis twice more: once with the requirement that charged tracks point to the event origin to within $2 \mathrm{~cm}$ in the $r-\phi$ plane, rather than $5 \mathrm{~cm}$ as in the standard analysis, and once with the restriction that the charged and neutral particles appear in the barrel region of the detector only, $\left|\cos \left(\theta_{\text {particle }}\right)\right|<0.7$.

Possible uncertainty related to the three-jet event definition was evaluated by repeating the analysis using a minimum of five particles per jet rather than two, and by repeating it once again requiring the angle between the highest energy jet in an event and the two lower energy ones to be $150 \pm 5^{\circ}$ rather than $150 \pm 10^{\circ}$.

Comparing the results obtained for these different conditions to the standard ones, systematic effects are observed only for the analysis using different values of the correction coefficients $q_{\text {n.mix }}$ and $q_{\text {g.tag }}$ and for that based on charged tracks alone. For the analysis based on charged tracks alone, the gluon jet fragmentation function is found to be systematically softer than in the standard analysis, for both the $k_{\perp}$ and cone jet definitions. No systematic trends are observed for the quark jet fragmentation function or for any of the other distributions, however. In contrast, the results obtained using Herwig instead of Jetset for detector corrections or the other criteria given above for track and cluster selection or to define a three jet event do not result in visible systematic deviations from the standard results for any of the distributions studied. Instead, bin-to-bin fluctuations above and below the standard results shown in Figs. 47, 10-15 and 17-18 are observed, which are small compared to the statistical uncertainties and which exhibit no clear systematic trend. The same is true for the calorimeter-only analysis employed for the $\psi_{\mathrm{E}}(r / R)$ and $\phi_{\mathrm{E}}(r / R)$ measurements (Figs. 10, 12 and 14).

Based on this information, systematic errors are assigned in the following manner. For the distributions involving measurements of the jet width (Figs. 4-5 and 10-15), two sources of systematic uncertainty are included: (1) the maximum difference between the standard results and those obtained using the extreme values of $q_{\text {n.mix }}$ and $q_{\text {g.tag }}$, and (2) the statistical uncertainty due to the detector correction factors. The total systematic uncertainty is defined by adding the two terms in quadrature for each bin of each distribution. The total uncertainties, given by the quadrature sum of the systematic and experimental statistical terms, are shown by the vertical error lines in Figs. 4-5 and 10-15. For each data point, the size of the experimental statistical error is indicated by the small horizontal bars. For the fragmentation functions (Figs. 6-7 and 17-18), three sources of systematic uncertainty are included: the two listed above, and (3) the difference between the standard results and those based on charged tracks alone. This third systematic term is added in quadrature with the other terms to define the total errors, shown in Figs. 6-7 and 17-18 by the vertical error lines. Numerical values for the total uncertainties of all these measurements are given in Tables 3-4 and 6-10. Systematic errors for the mean charged particle multiplicity values are evaluated in the same manner as for the fragmentation functions. The total systematic uncertainties for the multiplicity measurements are given in Tables 5 and 11 for the analyses which employ standard jet resolution parameters. For the ratio of the gluon to quark jet multiplicities, an explicit breakdown of the contributions 
of the various systematic terms is given in Table 12 .

As a last systematic check, the analysis was repeated using an alternative lifetime tagging algorithm to identify quark jets relative to that described in section 4 . Rather than use the decay length significance $L / \sigma_{L}$, an event was tagged if one of the two lower energy jets in the normal-mixture samples contained a reconstructed secondary vertex with a positive decay length $L$ between 0.15 and $0.50 \mathrm{~cm}$, with a decay length error $\sigma_{L}$ less than $0.10 \mathrm{~cm}$, while the other lower energy jet did not have a secondary vertex or else its decay length was less than $0.15 \mathrm{~cm} .{ }^{9}$ In addition, the number of tracks required to form a vertex was reduced to two, from the standard value of three: 4643 and 4324 tagged events were found using the $k_{\perp}$ and cone jet finders, respectively, with the standard jet resolution parameters. About $35 \%$ of these events are contained in the tagged samples found using the criteria of section 4 . The estimated purities of the anti-tagged gluon jets are $79.2 \%$ and $77.4 \%$ for the two jet finding methods. A check such as that described in section 5.3 was made between the Monte Carlo and data. The results are shown by the open triangles and dashed lines in Figs. 3(b) and (c). Good agreement between data and Monte Carlo is observed for the reconstructed secondary vertex rate in the highest energy jets of the tagged samples, compared to that observed for the normal-mixture samples, establishing a consistency check for the reliability of the Monte Carlo purity estimates. This alternative technique to obtain the tagged data sets therefore results in large differences in both the samples of events and the data corrections, compared to the standard analysis. Despite these differences, the effect on the results was in all cases found to be small. For example, the corrected results for the ratio of the gluon to quark jet mean charged particle multiplicities are found to be $\left\langle n_{k_{\perp}}^{\text {ch. }}\right\rangle_{\text {gluon }} /\left\langle n_{k_{\perp}}^{\text {ch. }}\right\rangle_{\text {quark }}=1.275 \pm 0.026$ (stat.) and $\left\langle n_{\text {cone }}^{\text {ch. }}\right\rangle_{\text {gluon }} /\left\langle n_{\text {cone }}^{\text {ch. }}\right\rangle_{\text {quark }}=1.113 \pm 0.023$ (stat. $)$, which agree with the standard results given in Tables 5 and 11 to well within the uncertainties. Therefore, we do not consider the technique chosen to identify the quark jets to represent an additional source of systematic error.

\section{Comparison to the gluon jet fragmentation function from $F_{L}(x)$ and $F_{T}(x)$}

Recently, OPAL has presented a study of the production angles of charged particles in inclusive hadronic $\mathrm{Z}^{0}$ events as a function of scaled particle energy $x=2 \cdot E / E_{c . m}$. [8]. From this information, we determined the longitudinal and transverse charged particle fragmentation functions, $F_{L}(x)$ and $F_{T}(x)$. By fitting to $F_{L}(x)$ and $F_{T}(x)$, a measurement of the charged particle fragmentation function of the gluon jet was extracted. This method to determine the gluon jet fragmentation function is therefore complementary to the method employed here, especially since it does not utilize a jet finder. The measurements obtained using this alternative technique are expected to be especially useful at small $x$ values, where the assignment of particles to a particular jet is highly dependent on the jet finding method.

In Fig. 23, we present a comparison of the gluon jet fragmentation function from [8] to those found in the present analysis using the standard jet resolution parameters. The results from the different methods are seen to be fairly consistent for values of $x_{E}$ larger than 0.20 . For $0.05<x_{E}<0.20$, where the results from $F_{L}(x)$ and $F_{T}(x)$ are expected to be most reliable,

\footnotetext{
${ }^{9}$ These criteria are the same as those chosen for our earlier publication [4].
} 
the fragmentation functions from the present analysis lie below that from [8]. Overall, the fragmentation function from $F_{L}(x)$ and $F_{T}(x)$ is seen to be softer than those of the present study. The differences between the distributions shown in Fig. 23 would be qualitatively consistent with a scaling violation if the data from [8] correspond to a larger mean jet energy scale than the value of $24 \mathrm{GeV}$ studied here. The calculations [30] used to extract the gluon fragmentation function from $F_{L}(x)$ and $F_{T}(x)$ are available to first order only, however, and as a consequence the jet energy scale to which those results are valid is unspecified. We naturally expect differences between the fragmentation function obtained using $F_{L}(x)$ and $F_{T}(x)$ and those obtained in this analysis because of the different theoretical and experimental definitions of the gluon jet.

\section{Discussion and summary}

In this paper, we have presented detailed measurements of quark and gluon jet properties and differences, based on the particle energy and multiplicity in jets. Our analysis employs a sample of several thousand tagged one-fold symmetric three jet events from $Z^{0}$ decays, in conjunction with a sample of over 50000 untagged symmetric events, to identify quark and gluon jets with energies of about $24 \mathrm{GeV}$ in an essentially unbiased and model independent manner. This work extends our investigations published in 1991 and 1993. The principal new feature of the present study is that we employ a cone jet finding algorithm similar to that used for $\mathrm{p} \overline{\mathrm{p}}$ experiments, in addition to the $k_{\perp}$ algorithm common for $\mathrm{e}^{+} \mathrm{e}^{-}$experiments. Thus, the measurements presented here should allow a direct comparison of $\mathrm{e}^{+} \mathrm{e}^{-}$and $\mathrm{p} \overline{\mathrm{p}}$ quark and gluon jet data, once an analysis using similar jet energies and cone jet resolution parameter values becomes available from the $\mathrm{p} \overline{\mathrm{p}}$ experiments. For the purposes of the present work, comparison of the results obtained using the two different jet finding methods permits us to assess the extent to which our conclusions depend on the jet definition. Within the cone and $k_{\perp}$ frameworks, we have also examined the sensitivity of the measurements to the values of the jet resolution parameters.

The results found using both the cone and $k_{\perp}$ jet finders demonstrate that gluon jets are substantially broader than quark jets, as measured from the distributions of particle energy or multiplicity around the jet axis. We also observe the charged particle fragmentation function, $\left(1 / N_{\text {event }}\right) \mathrm{d} n_{\text {ch. }} / \mathrm{d} x_{E}$, to be much softer for gluon jets than for quark jets, irrespective of the jet finding method. Within the $k_{\perp}$ framework, the results for quark and gluon jet properties are found to exhibit a fairly weak dependence on the choice of the resolution parameter, $y_{c u t}$, while the quark and gluon jet differences demonstrate almost no dependence on this choice. Within the cone framework, we similarly observe little dependence of the results on the minimum jet energy value, $\epsilon$. For the other resolution parameter of the cone algorithm, the cone size $R$, important differences are observed for different parameter choices. In general, the differences between quark and gluon jet properties become larger as $R$ increases, establishing the importance of soft particles at relatively large angles to the jet axes to the measured quark and gluon jet characteristics.

For the $k_{\perp}$ algorithm, we obtain $\left\langle n_{k_{\perp}}^{\text {ch. }}\right\rangle_{\text {gluon }} /\left\langle n_{k_{\perp}}^{\text {ch. }}\right\rangle_{\text {quark }}=1.251 \pm 0.024$ (stat.) \pm 0.029 (syst.) for the ratio of the gluon to quark jet mean charged particle multiplicity, essentially independent of the $y_{\text {cut }}$ value. For the cone algorithm, using a cone size of $R=30^{\circ}$, the corresponding result is $\left\langle n_{\text {cone }}^{\text {ch. }}\right\rangle_{\text {gluon }} /\left\langle n_{\text {cone }}^{\text {ch. }}\right\rangle_{\text {quark }}=1.096 \pm 0.023$ (stat.) \pm 0.023 (syst.), essentially independent of $\epsilon$, but this value increases to about 1.26 for a cone size of $R=50^{\circ}$. These differences in the 
results for the ratio value of gluon to quark jet particle multiplicity establish the importance of the jet definition to the interpretation of the experimental result and its comparison to QCD predictions, as has recently been emphasized in [27].

\section{Acknowledgements}

We thank Steve Ellis for many enjoyable and informative conversations concerning aspects of this analysis.

It is a pleasure to thank the SL Division for the efficient operation of the LEP accelerator and their continuing close cooperation with our experimental group. In addition to the support staff at our own institutions we are pleased to acknowledge the

Department of Energy, USA,

National Science Foundation, USA,

Particle Physics and Astronomy Research Council, UK,

Natural Sciences and Engineering Research Council, Canada, Fussefeld Foundation,

Israel Ministry of Science,

Israel Science Foundation, administered by the Israel Academy of Science and Humanities, Minerva Gesellschaft,

Japanese Ministry of Education, Science and Culture (the Monbusho) and a grant under the Monbusho International Science Research Program,

German Israeli Bi-national Science Foundation (GIF),

Direction des Sciences de la Matière du Commissariat à l'Energie Atomique, France,

Bundesministerium für Forschung und Technologie, Germany,

National Research Council of Canada,

A.P. Sloan Foundation and Junta Nacional de Investigação Científica e Tecnológica, Portugal. 


\section{References}

[1] S.J. Brodsky and J. Gunion, Phys. Rev. Lett. 37 (1976) 402;

K. Konishi, A. Ukawa and G. Veneziano, Phys. Lett. B78 (1978) 243;

K. Shizuya and S.-H.H. Tye, Phys. Rev. Lett. 41 (1978) 787;

M.B. Einhorn and B.G. Weeks, Nucl. Phys. B146 (1978) 445;

H.P. Nilles and K.H. Streng, Phys. Rev. D23 (1981) 1944;

A.H. Mueller, Nucl. Phys. B241 (1984) 141;

J.B. Gaffney and A.H. Mueller, Nucl. Phys. B250 (1985) 109;

E.D. Malaza and B.R. Webber, Nucl. Phys. B267 (1986) 702.

[2] JADE Collaboration, W. Bartel et al., Phys. Lett. 123B (1983) 460;

UA2 Collaboration, P. Bagnaia et al., Phys. Lett. 144B (1984) 291;

HRS Collaboration, M. Derrick et al., Phys. Lett. 165B (1985) 449;

MARK2 Collaboration, A. Petersen et al., Phys. Rev. Lett. 55 (1985) 1954;

UA1 Collaboration, G. Arnison et al., Nucl. Phys. B276 (1986) 253;

TASSO Collaboration, W. Braunschweig et al., Z. Phys. C45 (1989) 1;

AMY Collaboration, Y.K. Kim et al., Phys. Rev. Lett. 63 (1989) 1772;

CLEO Collaboration, M.S. Alam et al., Phys. Rev. D46 (1992) 4822;

VENUS Collaboration, H. Takaki et al., Phys. Rev. Lett. 71 (1993) 38.

[3] OPAL Collaboration, M.Z. Akrawy et al., Phys. Lett. B261 (1991) 334;

OPAL Collaboration, G. Alexander et al., Phys. Lett. B265 (1991) 462.

[4] OPAL Collaboration, P.D. Acton et al., Z. Phys. C58 (1993) 387.

[5] S. Catani et al., Phys. Lett. B269 (1991) 432;

N. Brown and W. J. Stirling, Z. Phys. C53 (1992) 629;

S. Bethke, Z. Kunszt, D. Soper and W.J. Stirling, Nucl. Phys. B370 (1992) 310.

[6] J.E. Huth et al., Research Directions for the Decade, Snowmass (1990), p.134, ed. E.L. Berger (World Scientific, Singapore);

CDF Collaboration, F. Abe et al., Phys. Rev. D45 (1992) 1448.

[7] OPAL Collaboration, R. Akers et al., Z. Phys. C63 (1994) 197.

[8] OPAL Collaboration, R. Akers et al., "Measurement of the longitudinal, transverse and asymmetry fragmentation functions at LEP", CERN-PPE/95-057.

[9] OPAL Collaboration, K. Ahmet et al., Nucl. Instr. and Meth. A305 (1991) 275.

[10] P. Allport et al., Nucl. Instr. and Meth. A324 (1993) 34;

P. Allport et al., Nucl. Instr. and Meth. A346 (1994) 476.

[11] M. Arignon et al., Nucl. Instr. and Meth. A313 (1992) 103.

[12] D. Charlton, F. Meijers, T. Smith and P. Wells, Nucl. Instr. and Meth. A325 (1993) 129; OPAL Collaboration, G. Alexander et al., Z. Phys. C52 (1991) 175.

[13] J.D. Bjorken and S.J. Brodsky, Phys. Rev. D1 (1970) 1416;

SLAC-LBL Collaboration, G. Hanson et al., Phys. Rev. Lett. 35 (1975) 1609. 
[14] OPAL Collaboration, P.D. Acton et al., Phys. Lett. B273 (1991) 355.

[15] T. Sjöstrand, Comp. Phys. Comm. 39 (1986) 347;

T. Sjöstrand and M. Bengtsson, Comp. Phys. Comm. 43 (1987) 367;

T. Sjöstrand, CERN-TH.6488/92.

[16] C. Peterson, D. Schlatter, I. Schmitt and P. Zerwas, Phys. Rev. D27 (1983) 105.

[17] J. Allison et al., Nucl. Instr. and Meth. A317 (1992) 47.

[18] G. Marchesini, B.R. Webber et al., Comp. Phys. Comm. 67 (1992) 465.

[19] Review of Particle Properties, Phys. Rev. D50 (1994) 1173.

[20] ALEPH Collaboration, D. Decamp et al., Phys. Lett. B244 (1990) 551;

L3 Collaboration, B. Adeva et al., Phys. Lett. B261 (1991) 177;

OPAL Collaboration, M.Z. Akrawy et al., Phys. Lett. B263 (1991) 311;

ALEPH Collaboration, D. Decamp et al., Phys. Lett. B266 (1991) 218;

DELPHI Collaboration, P. Abreu et al., Z. Phys. C56 (1992) 47.

[21] OPAL Collaboration, R. Akers et al., Z. Phys. C61 (1994) 209;

OPAL Collaboration, R. Akers et al., "A measurement of charged particle multiplicity in $Z^{0} \rightarrow \mathrm{c} \overline{\mathrm{c}}$ and $Z^{0} \rightarrow \mathrm{b} \overline{\mathrm{b}}$ events", CERN-PPE/95-038.

[22] D. Schaile in Proc. XXVII Int. Conf. on High Energy Physics, Glasgow 1994, ed. P.J. Bussey and I.G. Knowles (Institute of Physics Publishing, Bristol and Philadelphia);

T. Behnke and D.G. Charlton, "Electroweak measurements using heavy quarks at LEP", CERN-PPE/95-011, to be published in Physica Scripta.

[23] V. Khoze in Proc. Int. Symp. Lepton Photon Interactions, Stanford 1989, ed. M. Riordan (World Scientific, Singapore) 1990.

[24] L. Lönnblad, Comp. Phys. Comm. 71 (1992) 15.

[25] R. Odorico, Comp. Phys. Comm. 32 (1984) 139;

R. Odorico, Comp. Phys. Comm. 59 (1990) 527.

[26] OPAL Collaboration, M. Z. Akrawy et al., Z. Phys. C47 (1990) 505.

[27] J.W. Gary, Phys. Rev. D49 (1994) 4503.

[28] ALEPH Collaboration, D. Buskulic et al., Phys. Lett. B346 (1995) 389.

[29] CDF Collaboration, F. Abe et al., Phys. Rev. Lett. 70 (1993) 713.

[30] P. Nason and B.R. Webber, Nucl. Phys. B421 (1994) 473. 


\begin{tabular}{|c|c|c|}
\hline Systematic study & $k_{\perp}$ analysis & Cone analysis \\
\hline$q_{\text {g.tag }}$, central value and stat. error & $0.070 \pm 0.005$ (stat.) & $0.073 \pm 0.006$ (stat.) \\
\hline b lifetime & 0.008 & 0.014 \\
\hline $\mathrm{b}$ fragmentation function & 0.004 & 0.009 \\
\hline$\left\langle n_{\text {ch. }}\right\rangle_{\mathrm{B}}$ & 0.005 & 0.003 \\
\hline$\Gamma_{\mathrm{b} \overline{\mathrm{b}}} / \Gamma_{\text {hadron }}$ & 0.001 & 0.001 \\
\hline Impact parameter resolution & 0.009 & 0.012 \\
\hline Jet definition ambiguity & 0.002 & 0.011 \\
\hline Total systematic uncertainty & 0.014 & 0.023 \\
\hline
\end{tabular}

Table 1: Summary of systematic uncertainties for the gluon jet purity value, $q_{\text {g.tag }}$.

\begin{tabular}{|c|c|ccccc|}
\hline Sample & Condition & $\mathrm{d}$ & $\mathrm{u}$ & $\mathrm{s}$ & $\mathrm{c}$ & $\mathrm{b}$ \\
\hline \hline Normal-mixture & All events (100\%) & $22.3 \pm \mathbf{0 . 2}$ & $\mathbf{1 7 . 5} \pm \mathbf{0 . 1}$ & $22.1 \pm \mathbf{0 . 2}$ & $17.4 \pm 0.1$ & $20.7 \pm 0.1$ \\
\hline Tagged & All events (100\%) & $\mathbf{0 . 8 \pm 0 . 2}$ & $\mathbf{0 . 9} \pm \mathbf{0 . 2}$ & $1.7 \pm 0.2$ & $7.4 \pm 0.5$ & $89.2 \pm 0.6$ \\
\hline \hline \multirow{7}{*}{ Tagged } & $\begin{array}{c}\text { Correct gluon jet } \\
\text { identification, } G\left(z_{i}\right) \\
(93.0 \%)\end{array}$ & $0.3 \pm 0.1$ & $0.4 \pm 0.1$ & $0.6 \pm 0.2$ & $6.8 \pm 0.5$ & $91.9 \pm 0.5$ \\
\cline { 2 - 7 } & $\begin{array}{c}\text { Incorrect gluon jet } \\
\text { identification, } Q\left(z_{i}\right) \\
\text { (1) Gluon jet is the } \\
\text { highest energy jet (3.2\%) } \\
\text { (2) Gluon jet has the } \\
\text { vertex tag (3.8\%) }\end{array}$ & $12.5 \pm 3.2$ & $12.5 \pm 3.2$ & $28.8 \pm 4.4$ & $20.2 \pm 3.9$ & $26.0 \pm 4.3$ \\
\hline
\end{tabular}

Table 2: Flavor composition of the normal-mixture and tagged data samples as determined from the Jetset Monte Carlo including simulation of the detector. An explicit breakdown is also given for events with correctly and incorrectly identified gluon jets in the tagged sample (corresponding to $G\left(z_{i}\right)$ and $Q\left(z_{i}\right)$ in relation (3), respectively). The numbers in parentheses give the percentage of the sample satisfying the condition listed. The values shown are those found using the $k_{\perp}$ jet finder. Similar values are found using the cone jet finder. 


\begin{tabular}{|c|c|c|c|}
\hline$\chi$ & Gluon jet & Quark jet & Ratio \\
\hline $0^{\circ}-2^{\circ}$ & $0.0392 \pm 0.0041$ & $0.0871 \pm 0.0039$ & $0.450 \pm 0.063$ \\
\hline $2^{\circ}-4^{\circ}$ & $\mathbf{0 . 0 8 9 1 \pm \mathbf { 0 . 0 0 5 1 }}$ & $0.1548 \pm 0.0052$ & $0.575 \pm 0.049$ \\
\hline $4^{\circ}-6^{\circ}$ & $0.0963 \pm 0.0046$ & $0.1472 \pm 0.0049$ & $0.655 \pm 0.048$ \\
\hline $6^{\circ}-8^{\circ}$ & $0.1118 \pm 0.0046$ & $0.1070 \pm 0.0039$ & $1.045 \pm 0.074$ \\
\hline $8^{\circ}-10^{\circ}$ & $0.0883 \pm 0.0035$ & $0.0924 \pm 0.0035$ & $0.956 \pm 0.067$ \\
\hline $10^{\circ}-12^{\circ}$ & $0.0778 \pm 0.0030$ & $0.0720 \pm 0.0033$ & $1.081 \pm 0.084$ \\
\hline $12^{\circ}-14^{\circ}$ & $0.0672 \pm 0.0026$ & $0.0576 \pm 0.0026$ & $1.166 \pm 0.090$ \\
\hline $14^{\circ}-16^{\circ}$ & $0.0605 \pm 0.0024$ & $0.0440 \pm 0.0022$ & $1.37 \pm 0.11$ \\
\hline $16^{\circ}-18^{\circ}$ & $0.0494 \pm 0.0020$ & $0.0388 \pm 0.0022$ & $1.27 \pm 0.11$ \\
\hline $18^{\circ}-20^{\circ}$ & $0.0425 \pm 0.0018$ & $0.0319 \pm 0.0018$ & $1.33 \pm 0.12$ \\
\hline $20^{\circ}-24^{\circ}$ & $0.0346 \pm 0.0011$ & $0.0239 \pm 0.0012$ & $1.45 \pm 0.11$ \\
\hline $24^{\circ}-28^{\circ}$ & $0.02510 \pm 0.00090$ & $0.01632 \pm 0.00091$ & $1.54 \pm 0.13$ \\
\hline $28^{\circ}-32^{\circ}$ & $0.01939 \pm 0.00080$ & $0.01005 \pm 0.00066$ & $1.93 \pm 0.19$ \\
\hline $32^{\circ}-36^{\circ}$ & $0.01287 \pm 0.00059$ & $0.00797 \pm 0.00058$ & $1.62 \pm 0.18$ \\
\hline $36^{\circ}-44^{\circ}$ & $0.00887 \pm 0.00034$ & $0.00428 \pm 0.00031$ & $2.07 \pm 0.21$ \\
\hline $44^{\circ}-52^{\circ}$ & $0.00494 \pm 0.00024$ & $0.00304 \pm 0.00021$ & $1.62 \pm 0.17$ \\
\hline $52^{\circ}-60^{\circ}$ & $0.000335 \pm 0.00019$ & $0.00200 \pm 0.00018$ & $1.68 \pm 0.23$ \\
\hline
\end{tabular}

Table 3: The $\left(1 / E^{\text {jet }}\right)\left(\mathrm{d} E^{\text {jet }} / \mathrm{d} \chi\right) \mathrm{d} \chi$ distribution, obtained using the $k_{\perp}$ jet definition. The uncertainties include both the statistical and systematic terms. These data are displayed in Figs. 4 and 5. 


\begin{tabular}{|c|c|c|c|}
\hline$x_{E}$ & Gluon jet & Quark jet & Ratio \\
\hline $0.00-0.01$ & $61 \pm 45$ & $40.1 \pm 3.2$ & $1.5 \pm 1.1$ \\
\hline $0.01-0.02$ & $144.3 \pm 7.9$ & $105.4 \pm 3.5$ & $1.369 \pm \mathbf{0 . 0 8 3}$ \\
\hline $0.02-0.03$ & $119.6 \pm 5.0$ & $89.8 \pm 3.2$ & $1.332 \pm 0.094$ \\
\hline $0.03-0.04$ & $102.5 \pm 3.1$ & $66.0 \pm 3.0$ & $1.55 \pm 0.11$ \\
\hline $0.04-0.05$ & $80.2 \pm 3.0$ & $55.0 \pm 2.4$ & $1.46 \pm 0.11$ \\
\hline $0.05-0.06$ & $65.1 \pm 3.4$ & $45.0 \pm 2.6$ & $1.45 \pm 0.16$ \\
\hline $0.06-0.07$ & $50.7 \pm 2.2$ & $39.9 \pm 2.1$ & $1.27 \pm 0.11$ \\
\hline $0.07-0.08$ & $42.5 \pm 2.2$ & $32.1 \pm 1.8$ & $1.32 \pm 0.14$ \\
\hline $0.08-0.09$ & $35.4 \pm 1.8$ & $27.6 \pm 1.6$ & $1.29 \pm 0.13$ \\
\hline $0.09-0.10$ & $29.6 \pm 1.6$ & $24.3 \pm 1.5$ & $1.22 \pm 0.13$ \\
\hline $0.10-0.12$ & $22.81 \pm 0.95$ & $19.75 \pm 0.94$ & $1.155 \pm 0.094$ \\
\hline $0.12-0.14$ & $16.67 \pm 0.91$ & $15.3 \pm 1.1$ & $1.09 \pm 0.14$ \\
\hline $0.14-0.16$ & $12.06 \pm 0.71$ & $12.05 \pm 0.74$ & $1.00 \pm 0.11$ \\
\hline $0.16-0.18$ & $8.87 \pm 0.57$ & $9.68 \pm 0.62$ & $0.92 \pm 0.11$ \\
\hline $0.18-0.20$ & $7.00 \pm 0.53$ & $7.87 \pm 0.55$ & $0.89 \pm 0.12$ \\
\hline $0.20-0.25$ & $4.83 \pm 0.28$ & $5.18 \pm 0.27$ & $0.931 \pm 0.089$ \\
\hline $0.25-0.30$ & $2.37 \pm 0.18$ & $3.47 \pm 0.20$ & $0.683 \pm 0.082$ \\
\hline $0.30-0.40$ & $0.998 \pm 0.096$ & $1.87 \pm 0.10$ & $0.533 \pm 0.073$ \\
\hline $0.40-0.50$ & $0.414 \pm 0.065$ & $0.689 \pm 0.064$ & $0.60 \pm 0.13$ \\
\hline $0.50-0.60$ & $0.106 \pm 0.023$ & $0.347 \pm 0.031$ & $0.305 \pm 0.087$ \\
\hline $0.60-0.80$ & $0.032 \pm 0.011$ & $0.111 \pm 0.010$ & $0.29 \pm 0.11$ \\
\hline $0.80-1.00$ & - & $0.0212 \pm 0.0029$ & - \\
\hline
\end{tabular}

Table 4: The $\left(1 / N_{\text {event }}\right) \mathrm{d} n_{\text {ch. }} / \mathrm{d} x_{E}$ distribution, obtained using the $k_{\perp}$ jet definition. The uncertainties include both the statistical and systematic terms. These data are displayed in Figs. 6 and 7.

\begin{tabular}{|c|c|c|c|}
\hline \multirow{2}{*}{ jet definition } & $\left\langle n_{k_{\perp}}^{\text {ch. }}\right\rangle_{\text {gluon }}$ & $\left\langle n_{k_{\perp}}^{\text {ch. }}\right\rangle_{\text {quark }}$ & $\frac{\left\langle n_{k_{\perp}}^{\text {ch. }}\right\rangle_{\text {glnon }}}{\left\langle n_{k_{\perp}}^{\text {ch }}\right\rangle_{\text {quark }}}$ \\
\hline \hline$y_{\text {cut }}=\mathbf{0 . 0 2}$ & $9.10 \pm \mathbf{0 . 0 7} \pm \mathbf{0 . 0 9}$ & $7.27 \pm 0.07 \pm \mathbf{0 . 0 8}$ & $1.251 \pm 0.024 \pm \mathbf{0 . 0 2 9}$ \\
& $(1.073)$ & $(0.986)$ & $(1.088)$ \\
\hline$y_{\text {cut }}=\mathbf{0 . 0 0 5}$ & $8.83 \pm \mathbf{0 . 1 0}$ & $\mathbf{6 . 9 9} \pm \mathbf{0 . 0 9}$ & $1.262 \pm \mathbf{0 . 0 3 5}$ \\
& $(1.093)$ & $(0.986)$ & $(1.108)$ \\
\hline$y_{\text {cut }}=\mathbf{0 . 0 5}$ & $\mathbf{9 . 7 4} \pm \mathbf{0 . 1 2}$ & $7.48 \pm \mathbf{0 . 1 0}$ & $1.302 \pm \mathbf{0 . 0 4 1}$ \\
& $(1.076)$ & $(0.985)$ & $(1.092)$ \\
\hline
\end{tabular}

Table 5: Mean charged particle multiplicity values of jets defined using the $k_{\perp}$ jet finder. The numbers in parentheses are the detector correction factors. The uncertainties given for $y_{c u t}=0.02$ include both the experimental statistical (first error) and systematic (second error) terms. For the other $y_{c u t}$ choices, the uncertainties shown are the statistical terms from the experiment and detector correction factors, added in quadrature. These data are displayed in Fig. 9. 


\begin{tabular}{|c|c|c|c|}
\hline$r / R$ & Gluon jet & Quark jet & Ratio \\
\hline $0.000-0.067$ & $0.873 \pm 0.088$ & $1.578 \pm 0.075$ & $0.553 \pm 0.077$ \\
\hline $0.067-0.133$ & $1.620 \pm 0.096$ & $2.94 \pm 0.11$ & $0.551 \pm 0.049$ \\
\hline $0.133-0.200$ & $2.092 \pm 0.096$ & $2.346 \pm 0.085$ & $0.892 \pm 0.067$ \\
\hline $0.200-0.267$ & $1.829 \pm 0.077$ & $1.894 \pm 0.075$ & $0.966 \pm 0.072$ \\
\hline $0.267-0.333$ & $1.555 \pm 0.064$ & $1.433 \pm 0.065$ & $1.085 \pm 0.085$ \\
\hline $0.333-0.400$ & $1.393 \pm 0.058$ & $1.011 \pm 0.055$ & $1.38 \pm 0.12$ \\
\hline $0.400-0.467$ & $1.152 \pm 0.049$ & $0.808 \pm 0.044$ & $1.43 \pm 0.13$ \\
\hline $0.467-0.533$ & $0.891 \pm 0.039$ & $0.684 \pm 0.039$ & $1.30 \pm 0.12$ \\
\hline $0.533-0.600$ & $0.791 \pm 0.036$ & $0.525 \pm 0.039$ & $1.50 \pm 0.16$ \\
\hline $0.600-0.666$ & $0.680 \pm 0.033$ & $0.432 \pm 0.032$ & $1.57 \pm 0.18$ \\
\hline $0.666-0.733$ & $0.561 \pm 0.028$ & $0.390 \pm 0.031$ & $1.44 \pm 0.17$ \\
\hline $0.733-0.800$ & $0.523 \pm 0.028$ & $0.285 \pm 0.027$ & $1.84 \pm 0.25$ \\
\hline $0.800-0.867$ & $0.385 \pm 0.022$ & $0.296 \pm 0.023$ & $1.30 \pm 0.16$ \\
\hline $0.867-0.933$ & $0.382 \pm 0.023$ & $0.188 \pm 0.018$ & $2.03 \pm 0.29$ \\
\hline $0.933-1.000$ & $0.290 \pm 0.019$ & $0.162 \pm 0.022$ & $1.78 \pm 0.33$ \\
\hline
\end{tabular}

Table 6: The $\phi_{\mathrm{E}}(r / R)$ distribution, obtained using the cone jet definition. The uncertainties include both the statistical and systematic terms. These data are displayed in Figs. 10 and 14.

\begin{tabular}{|c|c|c|c|}
\hline$r / R$ & Gluon jet & Quark jet & Ratio \\
\hline $0.000-0.067$ & $0.380 \pm 0.042$ & $0.880 \pm 0.042$ & $0.432 \pm 0.065$ \\
\hline $0.067-0.133$ & $0.959 \pm 0.060$ & $1.778 \pm 0.067$ & $0.539 \pm 0.050$ \\
\hline $0.133-0.200$ & $1.365 \pm 0.069$ & $1.762 \pm 0.065$ & $0.774 \pm 0.062$ \\
\hline $0.200-0.267$ & $1.458 \pm 0.067$ & $1.613 \pm 0.064$ & $0.904 \pm 0.070$ \\
\hline $0.267-0.333$ & $1.354 \pm 0.064$ & $1.419 \pm 0.063$ & $0.954 \pm 0.079$ \\
\hline $0.333-0.400$ & $1.334 \pm 0.062$ & $1.208 \pm 0.064$ & $1.10 \pm 0.10$ \\
\hline $0.400-0.467$ & $1.275 \pm 0.062$ & $1.033 \pm 0.055$ & $1.23 \pm 0.11$ \\
\hline $0.467-0.533$ & $1.144 \pm 0.058$ & $0.915 \pm 0.057$ & $1.25 \pm 0.13$ \\
\hline $0.533-0.600$ & $1.029 \pm 0.053$ & $0.830 \pm 0.059$ & $1.24 \pm 0.14$ \\
\hline $0.600-0.666$ & $0.976 \pm 0.054$ & $0.740 \pm 0.052$ & $1.32 \pm 0.15$ \\
\hline $0.666-0.733$ & $0.868 \pm 0.051$ & $0.687 \pm 0.051$ & $1.26 \pm 0.15$ \\
\hline $0.733-0.800$ & $0.851 \pm 0.052$ & $0.565 \pm 0.049$ & $1.51 \pm 0.20$ \\
\hline $0.800-0.867$ & $0.649 \pm 0.041$ & $0.654 \pm 0.054$ & $0.99 \pm 0.13$ \\
\hline $0.867-0.933$ & $0.681 \pm 0.046$ & $0.439 \pm 0.042$ & $1.55 \pm 0.23$ \\
\hline $0.933-1.000$ & $0.537 \pm 0.039$ & $0.430 \pm 0.046$ & $1.25 \pm 0.20$ \\
\hline
\end{tabular}

Table 7: The $\phi_{\mathrm{M}}^{\text {ch. }}(r / R)$ distribution, obtained using the cone jet definition. The uncertainties include both the statistical and systematic terms. These data are displayed in Figs. 11 and 15. 


\begin{tabular}{|c|c|c|}
\hline$r / R$ & Gluon jet & Quark jet \\
\hline \hline $\mathbf{0 . 0 0 0 - 0 . 0 6 7}$ & $\mathbf{0 . 0 5 8 3} \pm \mathbf{0 . 0 0 5 9}$ & $\mathbf{0 . 1 0 5 3 \pm \mathbf { 0 . 0 0 5 0 }}$ \\
$\mathbf{0 . 0 6 7 - 0 . 1 3 3}$ & $\mathbf{0 . 1 6 5 8} \pm \mathbf{0 . 0 0 9 0}$ & $\mathbf{0 . 3 0 1 3} \pm \mathbf{0 . 0 0 9 5}$ \\
$\mathbf{0 . 1 3 3 - 0 . 2 0 0}$ & $\mathbf{0 . 3 0 6} \pm \mathbf{0 . 0 1 1}$ & $\mathbf{0 . 4 5 8} \pm \mathbf{0 . 0 1 2}$ \\
$\mathbf{0 . 2 0 0 - 0 . 2 6 7}$ & $\mathbf{0 . 4 2 7} \pm \mathbf{0 . 0 1 2}$ & $\mathbf{0 . 5 8 5} \pm \mathbf{0 . 0 1 3}$ \\
$\mathbf{0 . 2 6 7 - 0 . 3 3 3}$ & $\mathbf{0 . 5 3 1} \pm \mathbf{0 . 0 1 3}$ & $\mathbf{0 . 6 8 0} \pm \mathbf{0 . 0 1 3}$ \\
$\mathbf{0 . 3 3 3 - 0 . 4 0 0}$ & $\mathbf{0 . 6 2 4} \pm \mathbf{0 . 0 1 3}$ & $\mathbf{0 . 7 4 8} \pm \mathbf{0 . 0 1 3}$ \\
$\mathbf{0 . 4 0 0 - 0 . 4 6 7}$ & $\mathbf{0 . 7 0 0} \pm \mathbf{0 . 0 1 3}$ & $\mathbf{0 . 8 0 2} \pm \mathbf{0 . 0 1 3}$ \\
$\mathbf{0 . 4 6 7 - 0 . 5 3 3}$ & $\mathbf{0 . 7 6 0} \pm \mathbf{0 . 0 1 3}$ & $\mathbf{0 . 8 4 8} \pm \mathbf{0 . 0 1 3}$ \\
$\mathbf{0 . 5 3 3 - 0 . 6 0 0}$ & $\mathbf{0 . 8 1 2} \pm \mathbf{0 . 0 1 3}$ & $\mathbf{0 . 8 8 3} \pm \mathbf{0 . 0 1 3}$ \\
$\mathbf{0 . 6 0 0 - 0 . 6 6 6}$ & $\mathbf{0 . 8 5 8} \pm \mathbf{0 . 0 1 3}$ & $\mathbf{0 . 9 1 2} \pm \mathbf{0 . 0 1 3}$ \\
$\mathbf{0 . 6 6 6 - 0 . 7 3 3}$ & $\mathbf{0 . 8 9 5} \pm \mathbf{0 . 0 1 4}$ & $\mathbf{0 . 9 3 8} \pm \mathbf{0 . 0 1 3}$ \\
$\mathbf{0 . 7 3 3 - 0 . 8 0 0}$ & $\mathbf{0 . 9 3 0} \pm \mathbf{0 . 0 1 4}$ & $\mathbf{0 . 9 5 7} \pm \mathbf{0 . 0 1 3}$ \\
$\mathbf{0 . 8 0 0 - 0 . 8 6 7}$ & $\mathbf{0 . 9 5 5} \pm \mathbf{0 . 0 1 4}$ & $\mathbf{0 . 9 7 7} \pm \mathbf{0 . 0 1 3}$ \\
$\mathbf{0 . 8 6 7 - 0 . 9 3 3}$ & $\mathbf{0 . 9 8 1} \pm \mathbf{0 . 0 1 4}$ & $\mathbf{0 . 9 8 9} \pm \mathbf{0 . 0 1 3}$ \\
$\mathbf{0 . 9 3 3 - 1 . 0 0 0}$ & $1.000 \pm \mathbf{0 . 0 1 4}$ & $1.000 \pm \mathbf{0 . 0 1 4}$ \\
\hline
\end{tabular}

Table 8: The $\psi_{\mathrm{E}}(r / R)$ distribution, obtained using the cone jet definition. The uncertainties include both the statistical and systematic terms and are correlated from bin to bin. These data are displayed in Fig. 12.

\begin{tabular}{|c|c|c|}
\hline$r / R$ & Gluon jet & Quark jet \\
\hline $0.000-0.067$ & $\overline{00.0256 \pm 0.0028}$ & $\overline{0.0590 \pm 0.0028}$ \\
\hline $0.067-0.133$ & $0.0903 \pm 0.0053$ & $0.1782 \pm 0.0058$ \\
\hline $0.133-0.200$ & $0.1821 \pm 0.0073$ & $0.2964 \pm 0.0078$ \\
\hline $0.200-0.267$ & $0.2804 \pm 0.0087$ & $0.4045 \pm 0.0092$ \\
\hline $0.267-0.333$ & $0.3716 \pm 0.0098$ & $0.500 \pm 0.010$ \\
\hline $0.333-0.400$ & $0.461 \pm 0.011$ & $0.580 \pm 0.010$ \\
\hline $0.400-0.467$ & $0.547 \pm 0.011$ & $0.650 \pm 0.011$ \\
\hline $0.467-0.533$ & $0.624 \pm 0.012$ & $0.711 \pm 0.011$ \\
\hline $0.533-0.600$ & $0.693 \pm 0.012$ & $0.766 \pm 0.012$ \\
\hline $0.600-0.666$ & $0.759 \pm 0.013$ & $0.816 \pm 0.012$ \\
\hline $0.666-0.733$ & $0.817 \pm 0.013$ & $0.862 \pm 0.013$ \\
\hline $0.733-0.800$ & $0.875 \pm 0.013$ & $0.899 \pm 0.013$ \\
\hline $0.800-0.867$ & $0.918 \pm 0.014$ & $0.942 \pm 0.013$ \\
\hline $0.867-0.933$ & $0.964 \pm 0.014$ & $0.972 \pm 0.014$ \\
\hline 0.933-1.000 & $1.000 \pm 0.014$ & $1.000 \pm 0.014$ \\
\hline
\end{tabular}

Table 9: The $\psi_{\mathrm{M}}^{\text {ch. }}(r / R)$ distribution, obtained using the cone jet definition. The uncertainties include both the statistical and systematic terms and are correlated from bin to bin. These data are displayed in Fig. 13. 


\begin{tabular}{|c|c|c|c|}
\hline$x_{E}$ & Gluon jet & Quark jet & Ratio \\
\hline 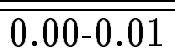 & $7.7 \pm 5.2$ & $\bar{~} 14.3 \pm 1.6$ & $\overline{0.54 \pm 0.39}$ \\
\hline $0.01-0.02$ & $42.1 \pm 3.3$ & $44.6 \pm 1.8$ & $0.943 \pm 0.094$ \\
\hline $0.02-0.03$ & $57.6 \pm 3.7$ & $48.8 \pm 2.1$ & $1.18 \pm 0.11$ \\
\hline $0.03-0.04$ & $55.4 \pm 2.8$ & $48.5 \pm 2.2$ & $1.143 \pm 0.095$ \\
\hline 0.04-0.05 & $54.0 \pm 2.7$ & $42.6 \pm 2.8$ & $1.27 \pm 0.13$ \\
\hline $0.05-0.06$ & $45.9 \pm 3.6$ & $40.7 \pm 2.7$ & $1.13 \pm 0.17$ \\
\hline $0.06-0.07$ & $43.5 \pm 2.2$ & $33.8 \pm 2.1$ & $1.29 \pm 0.13$ \\
\hline $0.07-0.08$ & $38.0 \pm 2.4$ & $28.7 \pm 1.8$ & $1.33 \pm 0.15$ \\
\hline $0.08-0.09$ & $32.8 \pm 2.2$ & $25.8 \pm 1.6$ & $1.27 \pm 0.15$ \\
\hline $0.09-0.10$ & $27.7 \pm 2.2$ & $24.3 \pm 2.0$ & $1.14 \pm 0.18$ \\
\hline $0.10-0.12$ & $24.2 \pm 1.2$ & $19.5 \pm 1.1$ & $1.24 \pm 0.12$ \\
\hline $0.12-0.14$ & $17.66 \pm 0.95$ & $15.88 \pm 0.86$ & $1.11 \pm 0.11$ \\
\hline $0.14-0.16$ & $14.14 \pm 0.92$ & $12.33 \pm 0.83$ & $1.15 \pm 0.14$ \\
\hline $0.16-0.18$ & $10.05 \pm 0.89$ & $11.0 \pm 1.0$ & $0.92 \pm 0.17$ \\
\hline $0.18-0.20$ & $8.99 \pm 0.67$ & $7.78 \pm 0.58$ & $1.16 \pm 0.16$ \\
\hline $0.20-0.25$ & $5.48 \pm 0.32$ & $6.35 \pm 0.44$ & $0.86 \pm 0.10$ \\
\hline $0.25-0.30$ & $3.01 \pm 0.25$ & $4.20 \pm 0.34$ & $0.72 \pm 0.12$ \\
\hline $0.30-0.40$ & $1.66 \pm 0.17$ & $1.93 \pm 0.16$ & $0.86 \pm 0.14$ \\
\hline $0.40-0.50$ & $0.656 \pm 0.096$ & $0.809 \pm 0.069$ & $0.81 \pm 0.17$ \\
\hline $0.50-0.60$ & $0.197 \pm 0.041$ & $0.400 \pm 0.040$ & $0.49 \pm 0.14$ \\
\hline $0.60-0.80$ & $0.049 \pm 0.013$ & $0.152 \pm 0.015$ & $0.32 \pm 0.11$ \\
\hline $0.80-1.00$ & $0.0064 \pm 0.0054$ & $0.0247 \pm 0.0055$ & $0.26 \pm 0.25$ \\
\hline
\end{tabular}

Table 10: The $\left(1 / N_{\text {event }}\right) \mathrm{d} n_{\text {ch. }} / \mathrm{d} x_{E}$ distribution, obtained using the cone jet definition. The uncertainties include both the statistical and systematic terms. These data are displayed in Figs. 17 and 18.

\begin{tabular}{|c|c|c|c|}
\hline jet definition & $\left\langle n_{\text {cone }}^{\text {ch. }}\right\rangle_{\text {gluon }}$ & $\left\langle n_{\text {cone }}^{\text {ch. }}\right\rangle_{\text {quark }}$ & $\frac{\left\langle n_{\text {cone }}^{\text {ch }}\right\rangle_{\text {glnon }}}{\left\langle n_{\text {cone }}^{\text {ch }}\right\rangle_{\text {quark }}}$ \\
\hline \hline$R=30^{\circ}, \epsilon=10 \mathrm{GeV}$ & $6.26 \pm \mathbf{0 . 0 6} \pm 0.07$ & $5.71 \pm \mathbf{0 . 0 5} 0.05$ & $1.096 \pm \mathbf{0 . 0 2 3 \pm 0 . 0 2 3}$ \\
& $(1.031)$ & $(0.944)$ & $(1.092)$ \\
\hline$R=40^{\circ}, \epsilon=10 \mathrm{GeV}$ & $7.43 \pm 0.09$ & $6.44 \pm 0.08$ & $1.153 \pm \mathbf{0 . 0 3 2}$ \\
& $(1.055)$ & $(0.959)$ & $(1.100)$ \\
\hline$R=50^{\circ}, \epsilon=10 \mathrm{GeV}$ & $8.75 \pm \mathbf{0 . 1 7}$ & $6.95 \pm 0.16$ & $1.257 \pm \mathbf{0 . 0 6 1}$ \\
& $(1.118)$ & $(0.962)$ & $(1.161)$ \\
\hline$R=30^{\circ}, \epsilon=5 \mathrm{GeV}$ & $6.37 \pm \mathbf{0 . 0 8}$ & $5.68 \pm 0.07$ & $1.123 \pm \mathbf{0 . 0 3 1}$ \\
& $(1.064)$ & $(0.942)$ & $(1.130)$ \\
\hline$R=30^{\circ}, \epsilon=15 \mathrm{GeV}$ & $6.50 \pm 0.08$ & $5.62 \pm 0.08$ & $1.156 \pm \mathbf{0 . 0 3 4}$ \\
& $(0.998)$ & $(0.919)$ & $(1.085)$ \\
\hline
\end{tabular}

Table 11: Mean charged particle multiplicity values of jets defined using the cone jet finder. The numbers in parentheses are the detector correction factors. The uncertainties given for $R=30^{\circ}$, $\epsilon=10 \mathrm{GeV}$ include both the experimental statistical (first error) and systematic (second error) terms. For the other jet resolution choices, the uncertainties shown are the statistical terms from the experiment and detector correction factors, added in quadrature. These data are displayed in Fig. 22 . 


\begin{tabular}{|c|c|c|}
\hline Systematic study & $\begin{array}{c}k_{\perp} \text { analysis, } \\
y_{\text {cut }}=0.02 \\
\Delta\left(\frac{\left\langle n_{k_{\perp}}^{\text {ch. }}\right\rangle_{\text {glnon }}}{\left\langle n_{k_{\perp}}^{\text {ch }}\right\rangle_{\text {qna rk }}}\right)\end{array}$ & $\begin{array}{c}\text { Cone analysis, } \\
R=30^{\circ}, \epsilon=10 \mathrm{GeV} \\
\Delta\left(\frac{\left\langle n_{\text {conen }}^{\text {ch }}\right\rangle_{\text {glnon }}}{\left\langle n_{\text {cone }}^{\text {ch }}\right\rangle_{\text {quark }}}\right)\end{array}$ \\
\hline $\begin{array}{c}\left\langle n^{\text {ch. }}\right\rangle_{\text {gluon }} /\left\langle n^{\text {ch. }}\right\rangle_{\text {quark }} \\
\text { central value and stat. error }\end{array}$ & $\bar{~} 1.251 \pm 0.024$ (stat.) & $1.096 \pm 0.023$ (stat.) \\
\hline $\begin{array}{l}\text { Correction coeffs. } \\
\left(q_{\text {n.mix }} \text { and } q_{\text {g.tag }}\right)\end{array}$ & 0.010 & 0.001 \\
\hline Charged particles only & 0.021 & 0.018 \\
\hline Monte Carlo statistics & 0.018 & 0.014 \\
\hline Total systematic unce & 0.029 & 0.023 \\
\hline
\end{tabular}

Table 12: Breakdown of the contributions to the systematic uncertainty for the ratios of mean charged particle multiplicity between gluon and quark jets. 

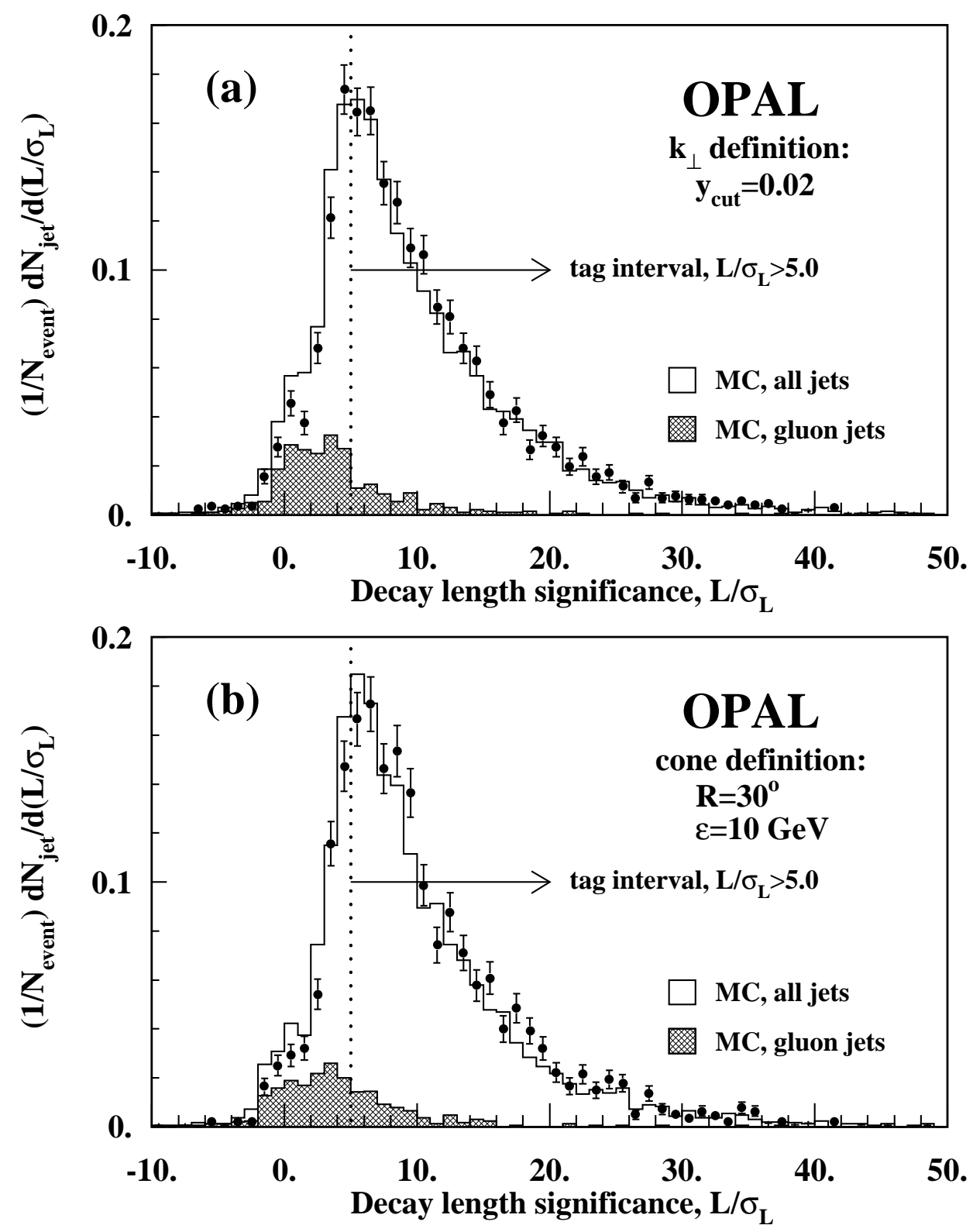

Figure 1: (a) Decay length significance distribution for the two lower energy jets of the normalmixture sample, defined using the $k_{\perp}$ jet finder, compared to the prediction of the Jetset Monte Carlo including detector simulation, after the cuts described in the text have been applied. The contribution of gluon jets to the Monte Carlo distribution is shown by the filled area. (b) Corresponding distribution for the cone jet finder. 


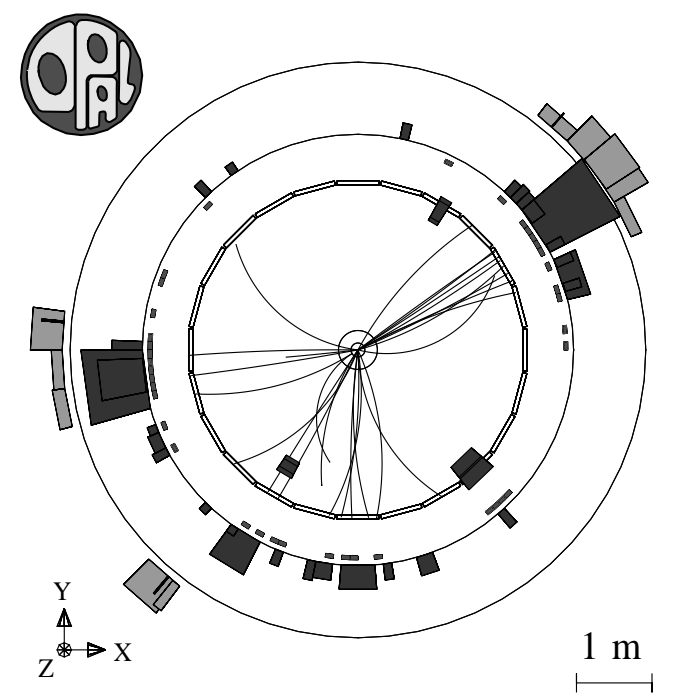

(a)

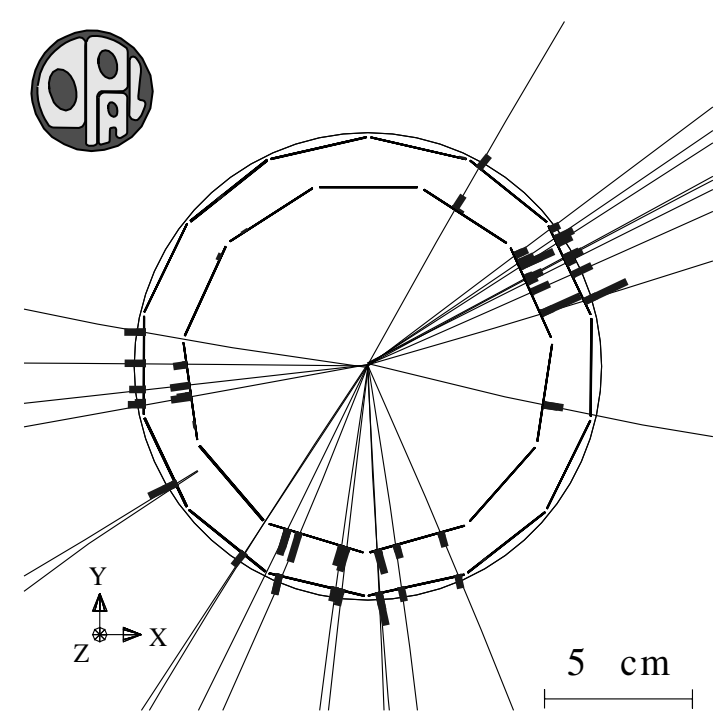

(b)

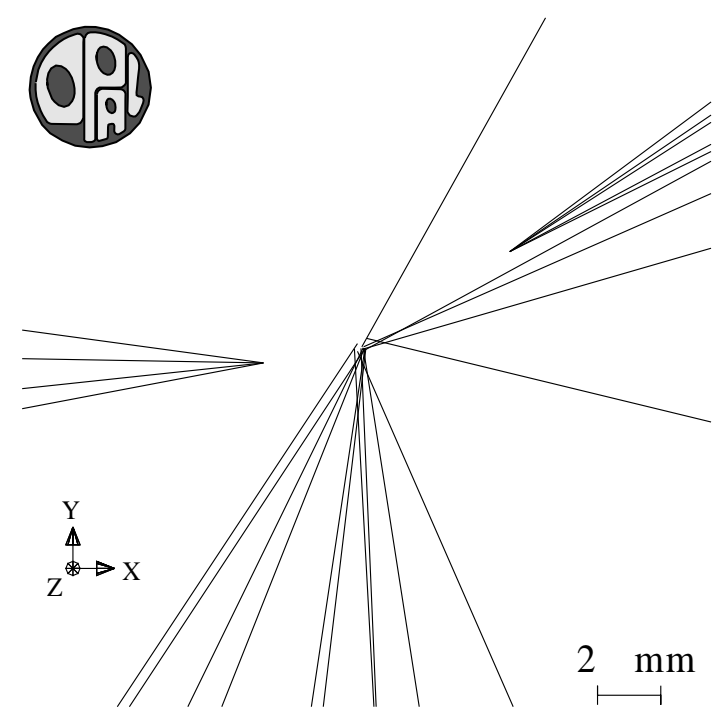

(c)

Figure 2: (a) $r-\phi$ view of a one-fold symmetric three jet event of the type used in this analysis (OPAL data event 207191 from Run 5267 , collected in July 1994), showing the charged tracks reconstructed in the central detector and the energy deposits in the electromagnetic and hadronic calorimeters. (b) Enlarged view showing the tracks and hits in the two layers of the silicon microvertex detector. (c) Enlarged view showing the tracks extrapolated to the region around the $\mathrm{e}^{+} \mathrm{e}^{-}$collision point. 


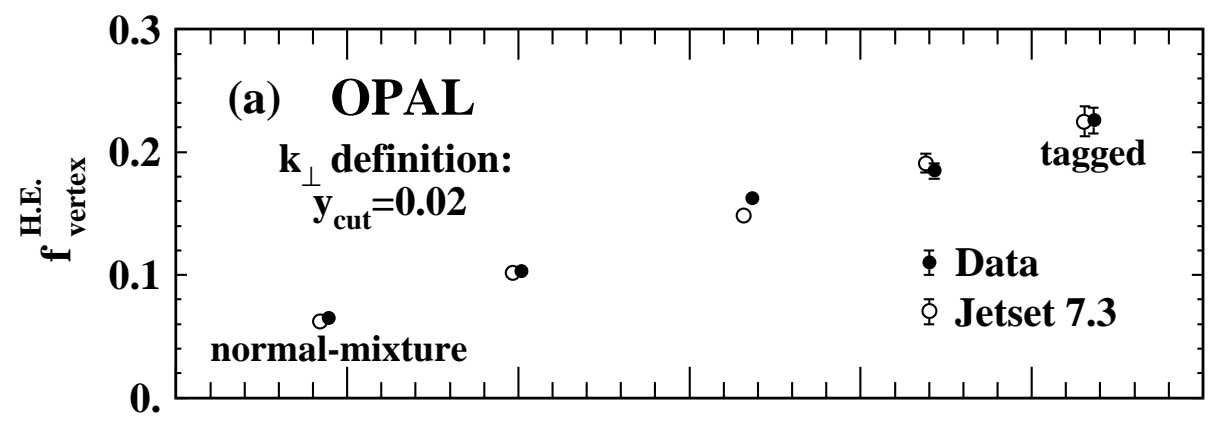

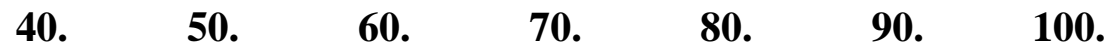

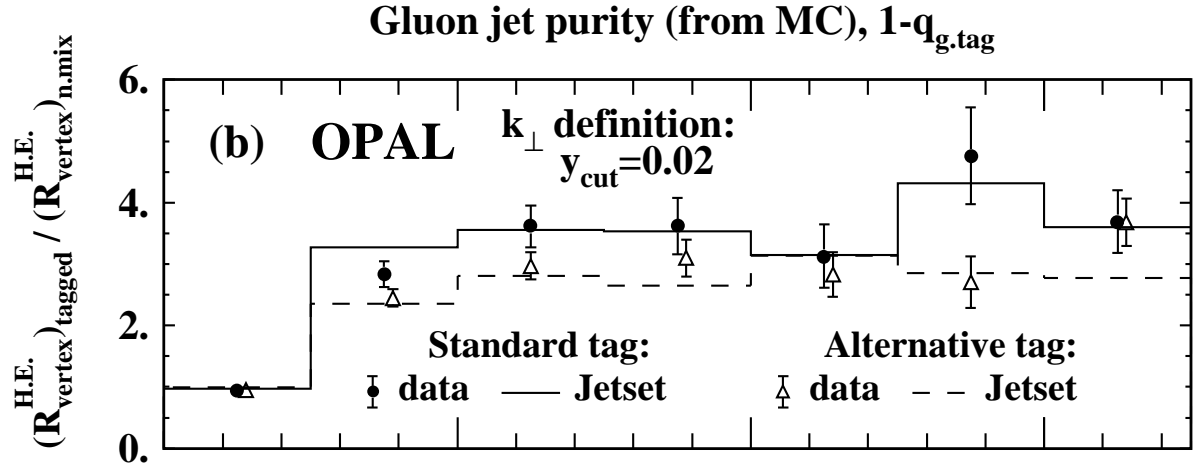

0. $10 . \quad 20 . \quad 30$.

Decay length significance, $L / \sigma_{L}$

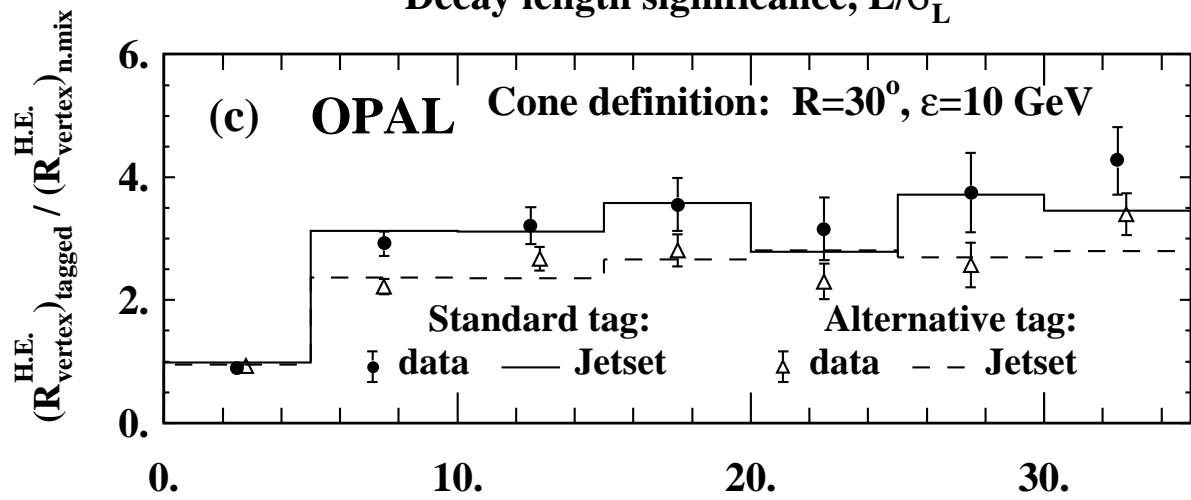

Decay length significance, $L / \sigma_{L}$

Figure 3: (a): The rate at which secondary vertices with $L / \sigma_{L}>5$ are found in the highest energy (H.E.) jets, as the b quark tagging conditions in the lower energy jets are varied, versus the corresponding Monte Carlo value for the gluon jet purity; the results shown are found using the $k_{\perp}$ jet finder. (b) and (c): The ratio of the rates at which secondary vertices are reconstructed in the highest energy jets of the tagged sample, to the corresponding quantity from the normal-mixture sample, displayed in differential bins of $L / \sigma_{L}$ in the highest energy jets for the $k_{\perp}$ and cone jet definitions. Two different samples of tagged events are employed for this study, as discussed in the text. 


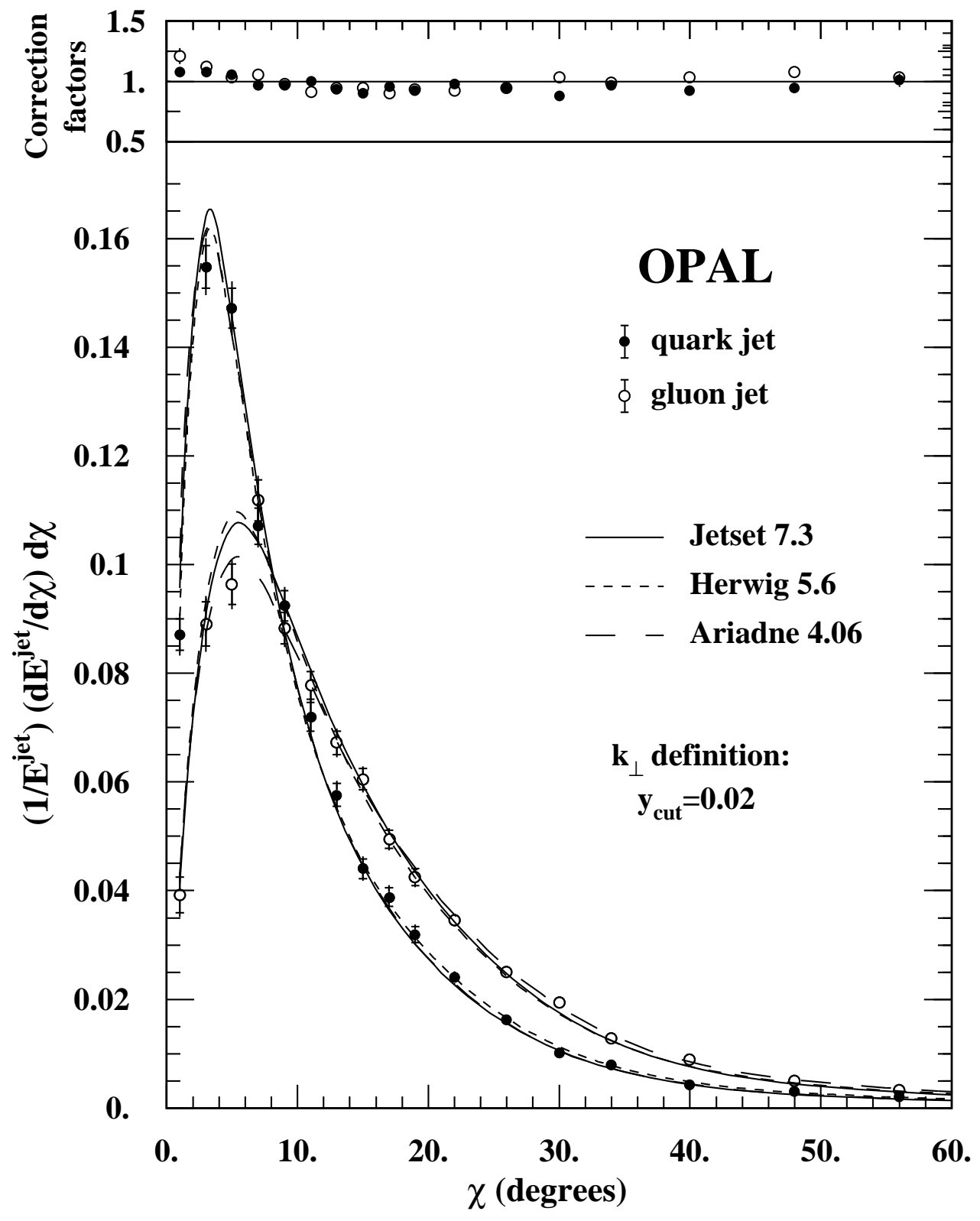

Figure 4: The normalized distribution of jet energy with respect to the jet axis for quark and gluon jets defined using the $k_{\perp}$ jet finder; $\chi$ is the angle of a particle with respect to the jet axis. The data have been corrected for quark and gluon jet misidentification and for the effects of the detector. The errors shown include both the statistical and systematic terms; the experimental statistical uncertainties are indicated by the small horizontal bars. The detector correction factors are shown in the small figure above the data distributions. Also shown are the predictions of the Jetset, Herwig and Ariadne QCD shower models. These data are tabulated in Table 3. 


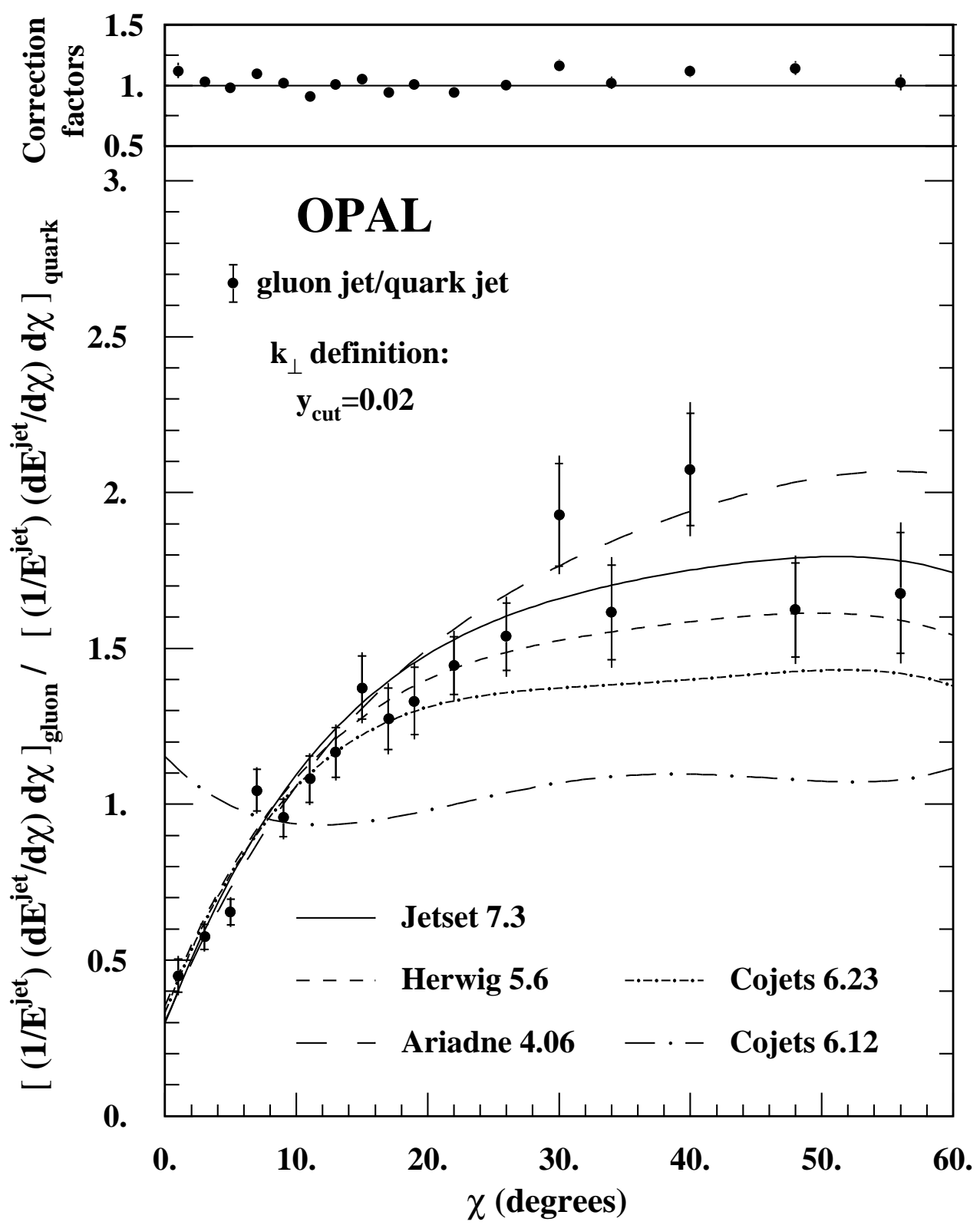

Figure 5: The ratio of the gluon to quark jet measurements for the data shown in Fig. 4. The errors shown include both the statistical and systematic terms; the experimental statistical uncertainties are indicated by the small horizontal bars. The predictions of the Cojets QCD shower model are shown along with those of Jetset, Herwig and Ariadne. These data are tabulated in Table 3. 


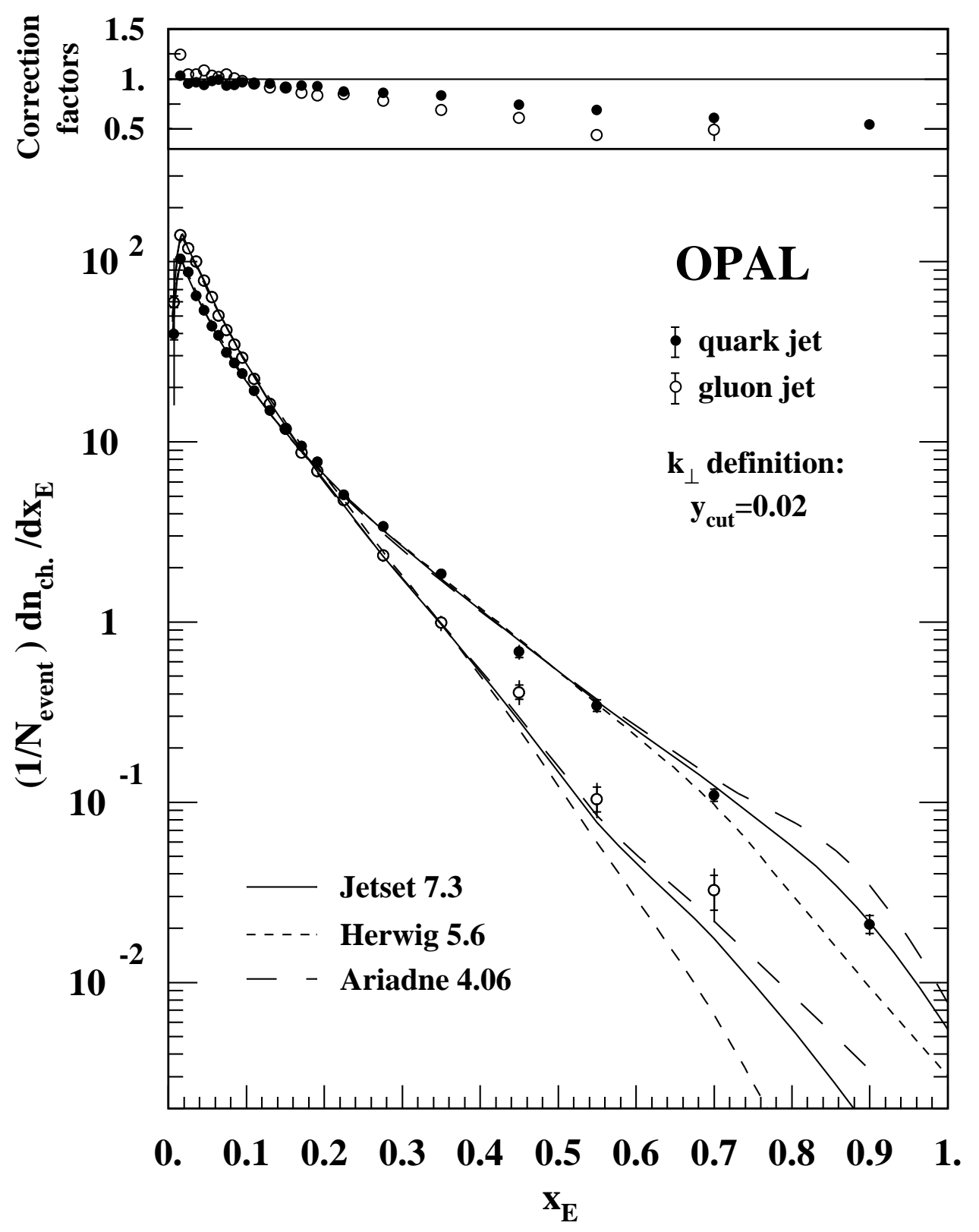

Figure 6: Charged particle fragmentation functions of quark and gluon jets defined using the $k_{\perp}$ jet finder. The errors shown include both the statistical and systematic terms; the experimental statistical uncertainties are indicated by the small horizontal bars. These data are tabulated in Table 4. 


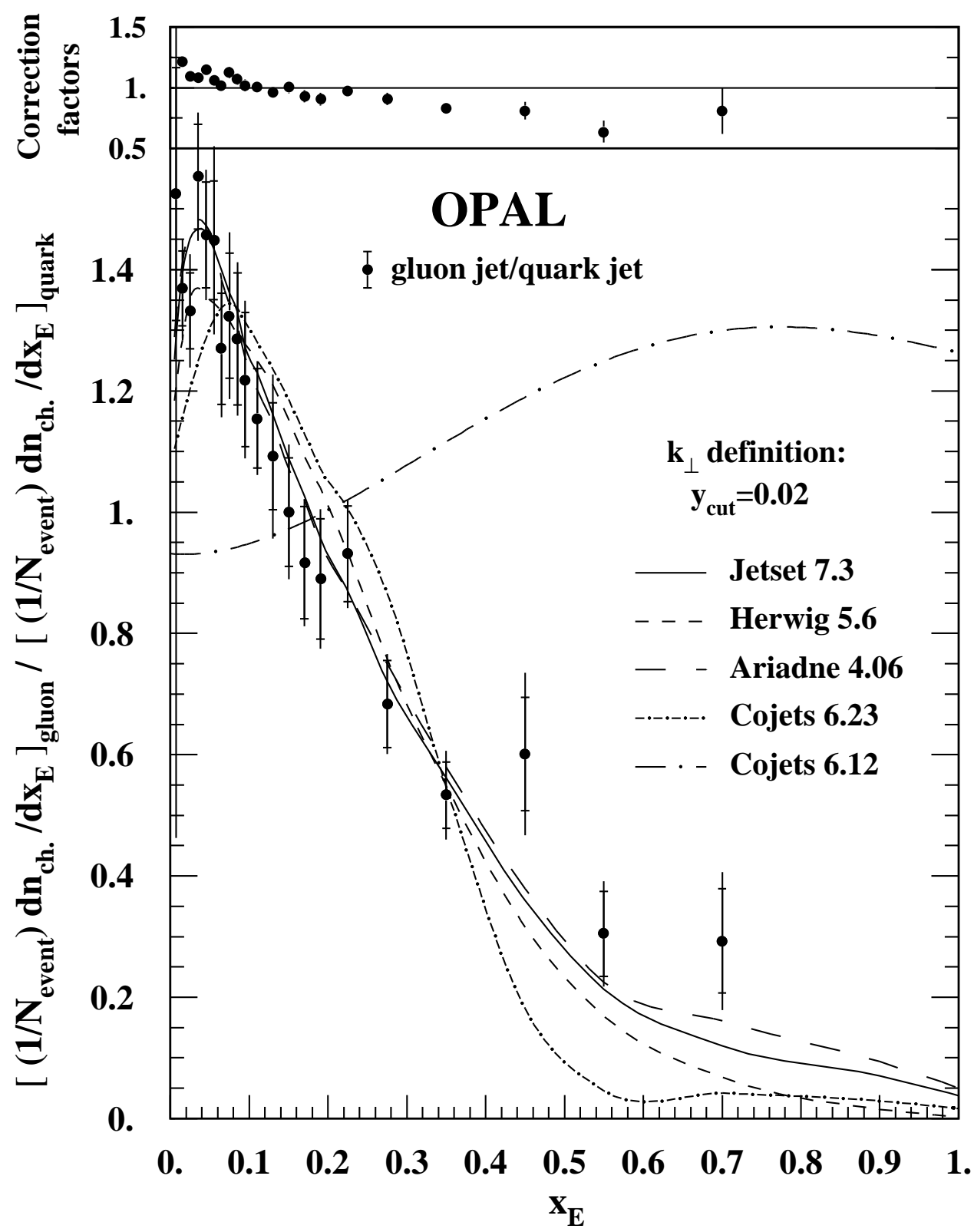

Figure 7: The ratio of the distributions shown in Fig. 6. The errors shown include both the statistical and systematic terms; the experimental statistical uncertainties are indicated by the small horizontal bars. These data are tabulated in Table 4. 

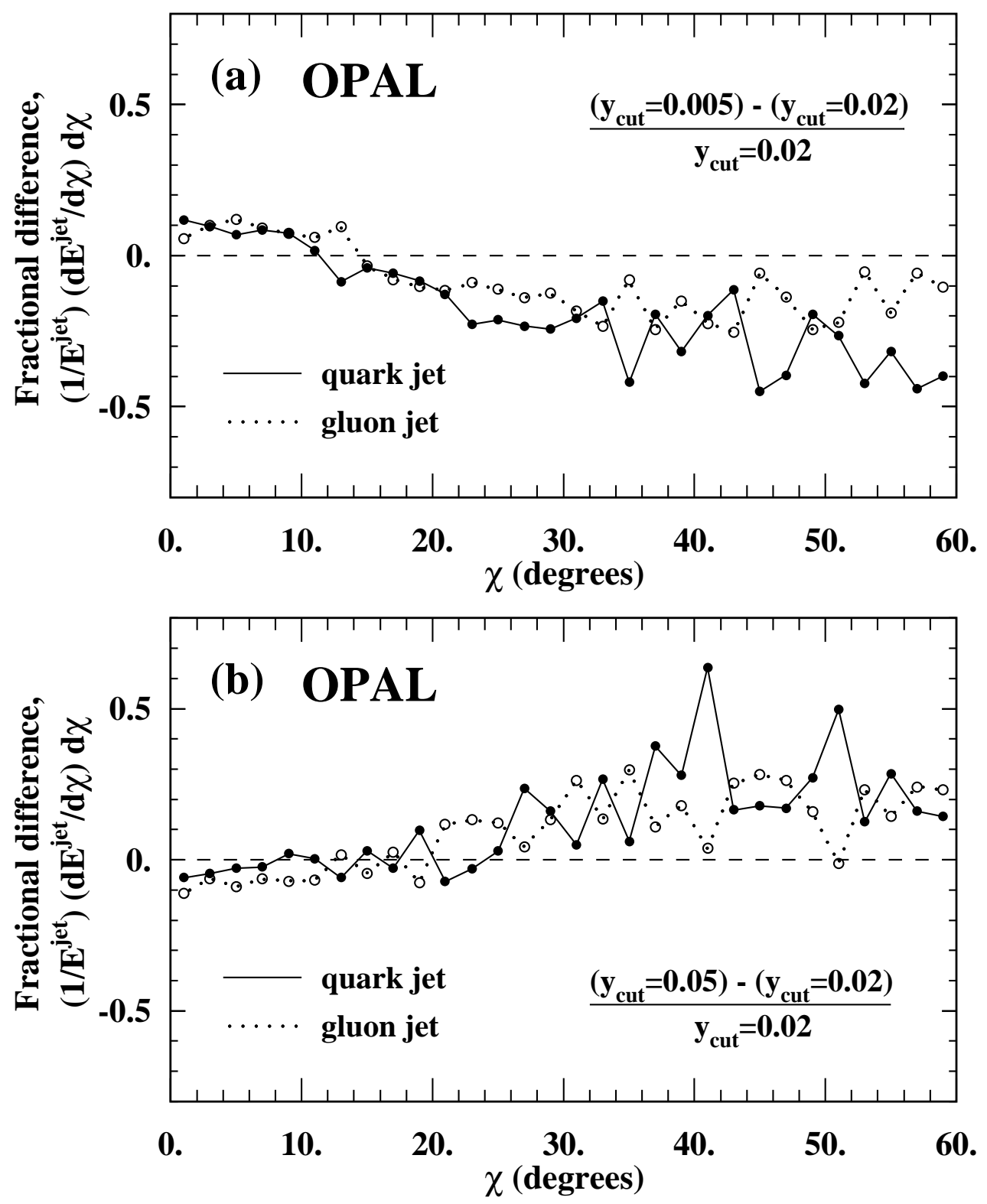

Figure 8: Fractional difference between the normalized jet energy distributions of quark and gluon jets defined using (a) $y_{\text {cut }}=0.005$ and $y_{\text {cut }}=0.02$, and (b) $y_{\text {cut }}=0.05$ and $y_{\text {cut }}=0.02$, using the $k_{\perp}$ jet finder. The point to point fluctuations in the curves indicate the level of statistical uncertainty. 

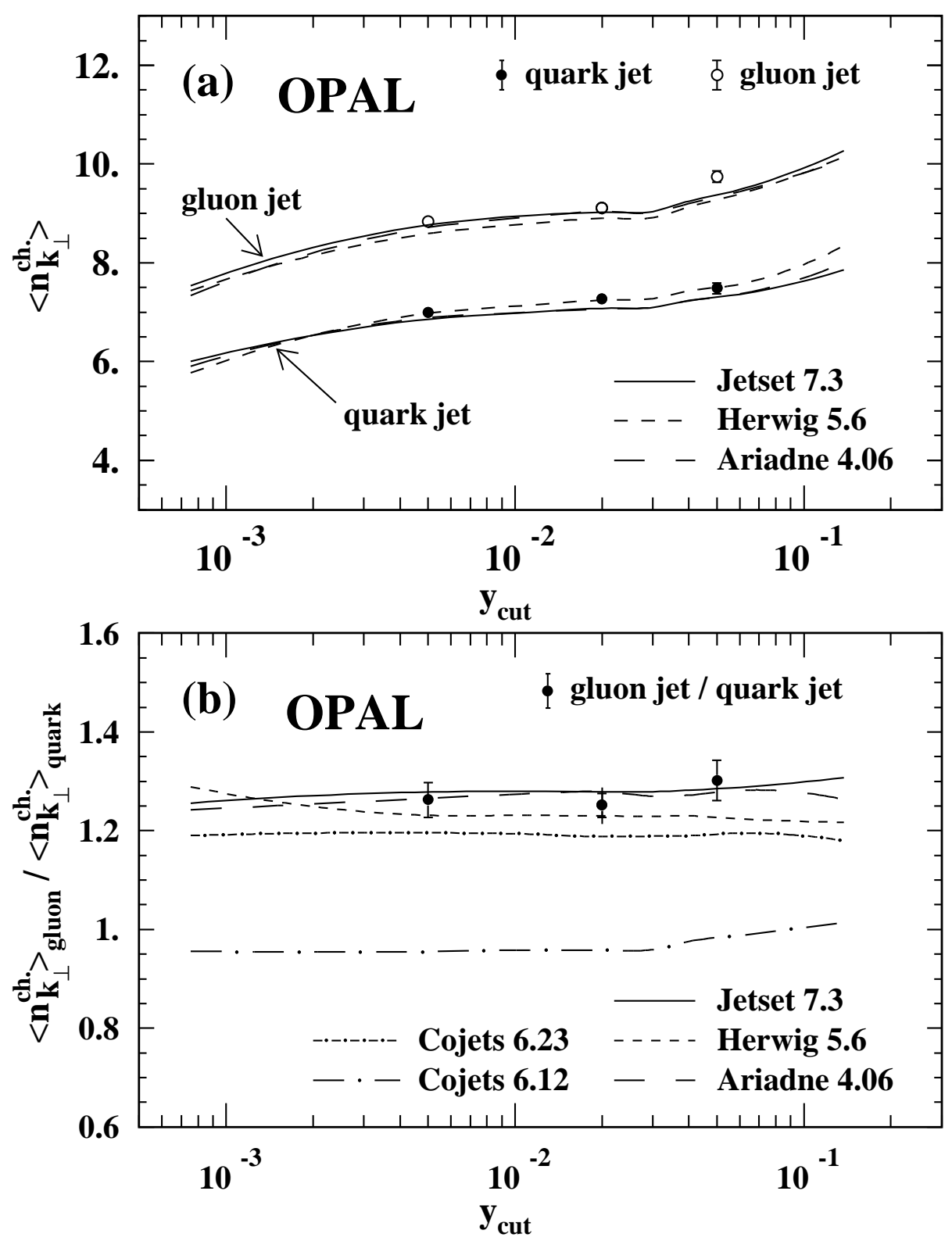

Figure 9: (a) Mean charged multiplicity values of gluon and quark jets defined using the $k_{\perp}$ jet finder with different $y_{\text {cut }}$ values. (b) Ratio of the mean charged multiplicity values of gluon to quark jets as a function of $y_{\text {cut }}$. For the results based on $y_{\text {cut }}=0.02$, the total errors including statistical and systematic terms are shown; the size of the experimental statistical uncertainties are indicated by the small horizontal bars. For the other $y_{\text {cut }}$ values, the uncertainties shown are the statistical terms from the experiment and detector correction factors, added in quadrature. These data are tabulated in Table 5 . 


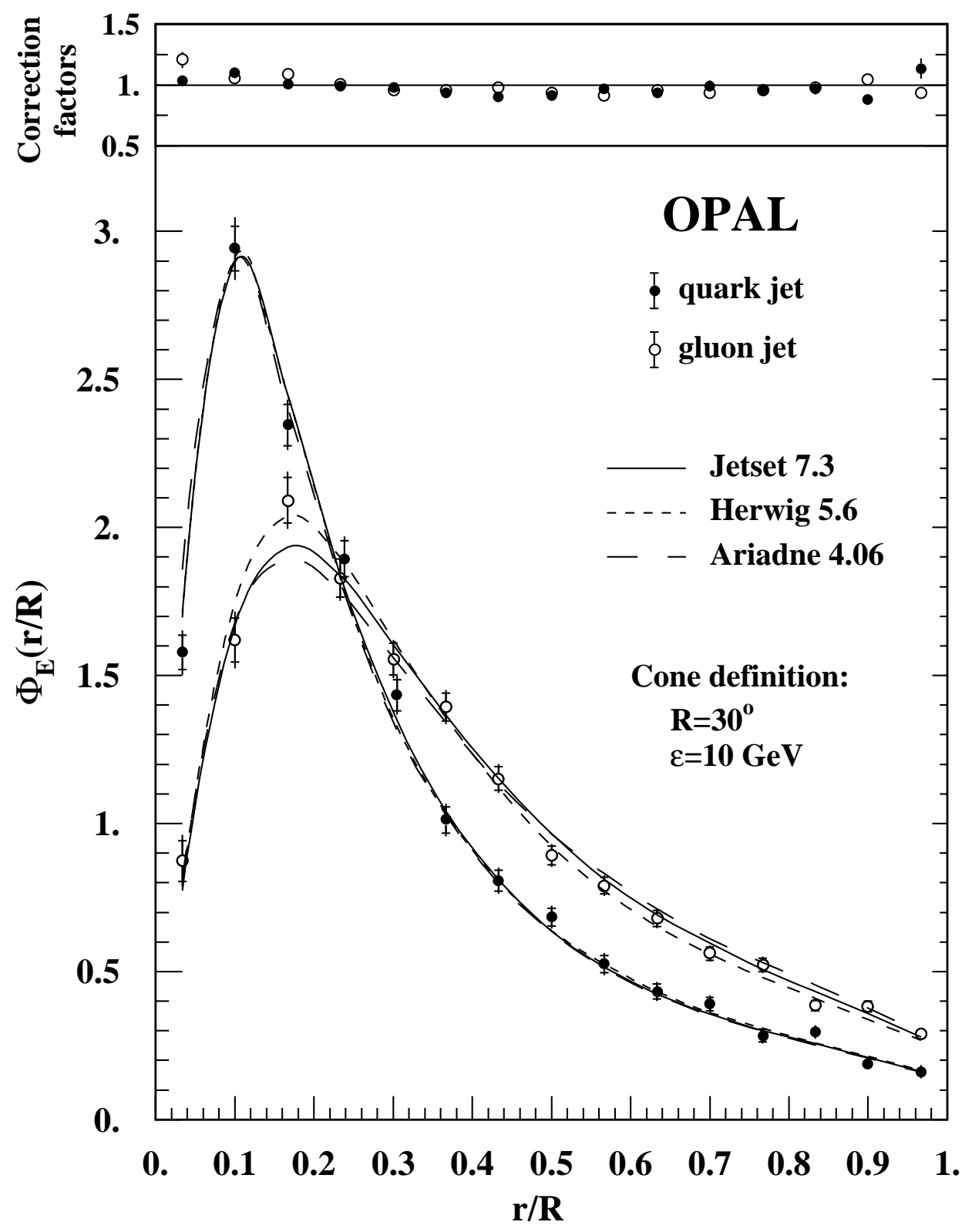

Figure 10: The differential energy profile of gluon and quark jets defined using the cone algorithm. The errors shown include both the statistical and systematic terms; the experimental statistical uncertainties are indicated by the small horizontal bars. These data are tabulated in Table 6 . 


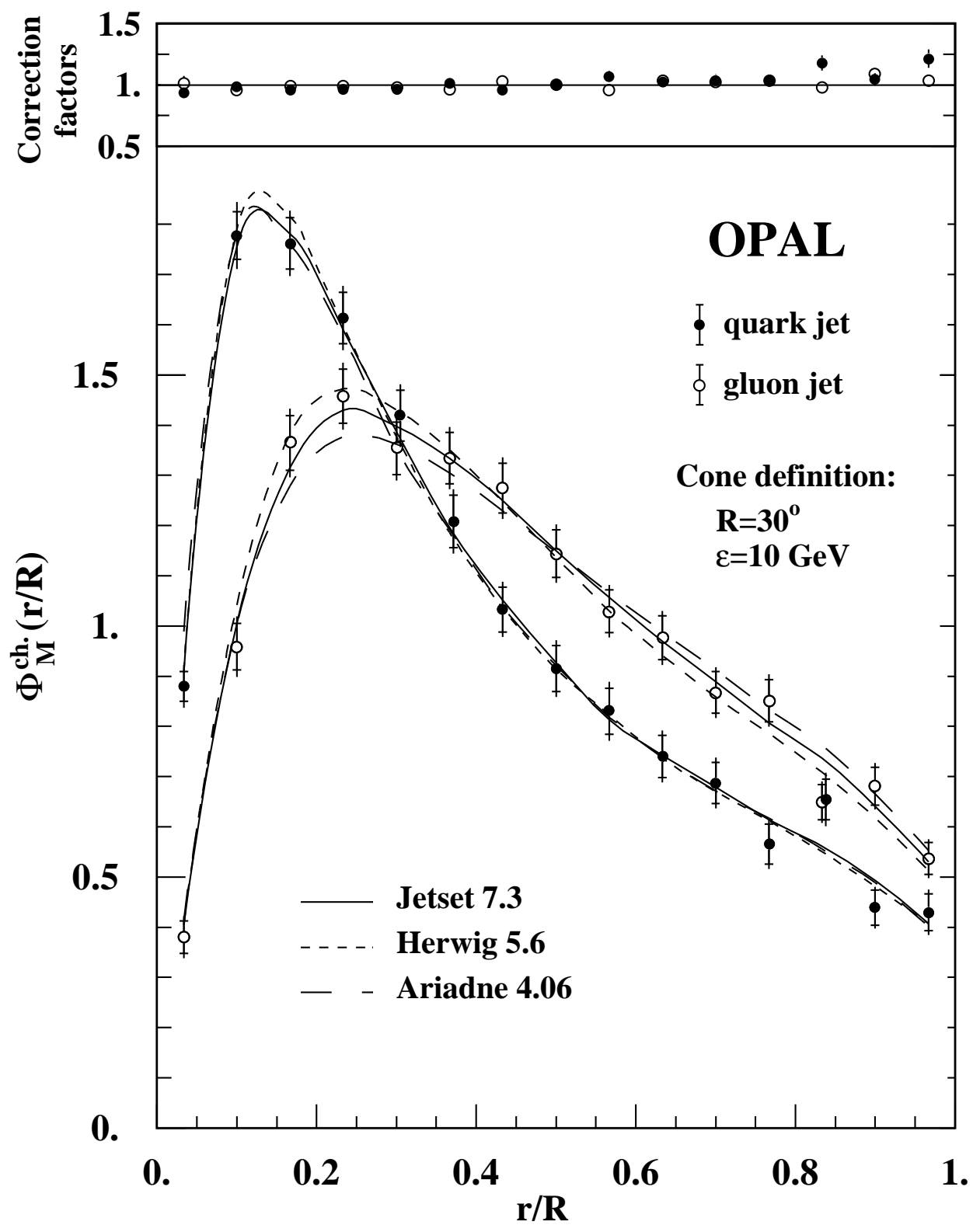

Figure 11: The differential charged particle multiplicity profile of gluon and quark jets defined using the cone algorithm. The errors shown include both the statistical and systematic terms; the experimental statistical uncertainties are indicated by the small horizontal bars. These data are tabulated in Table 7. 


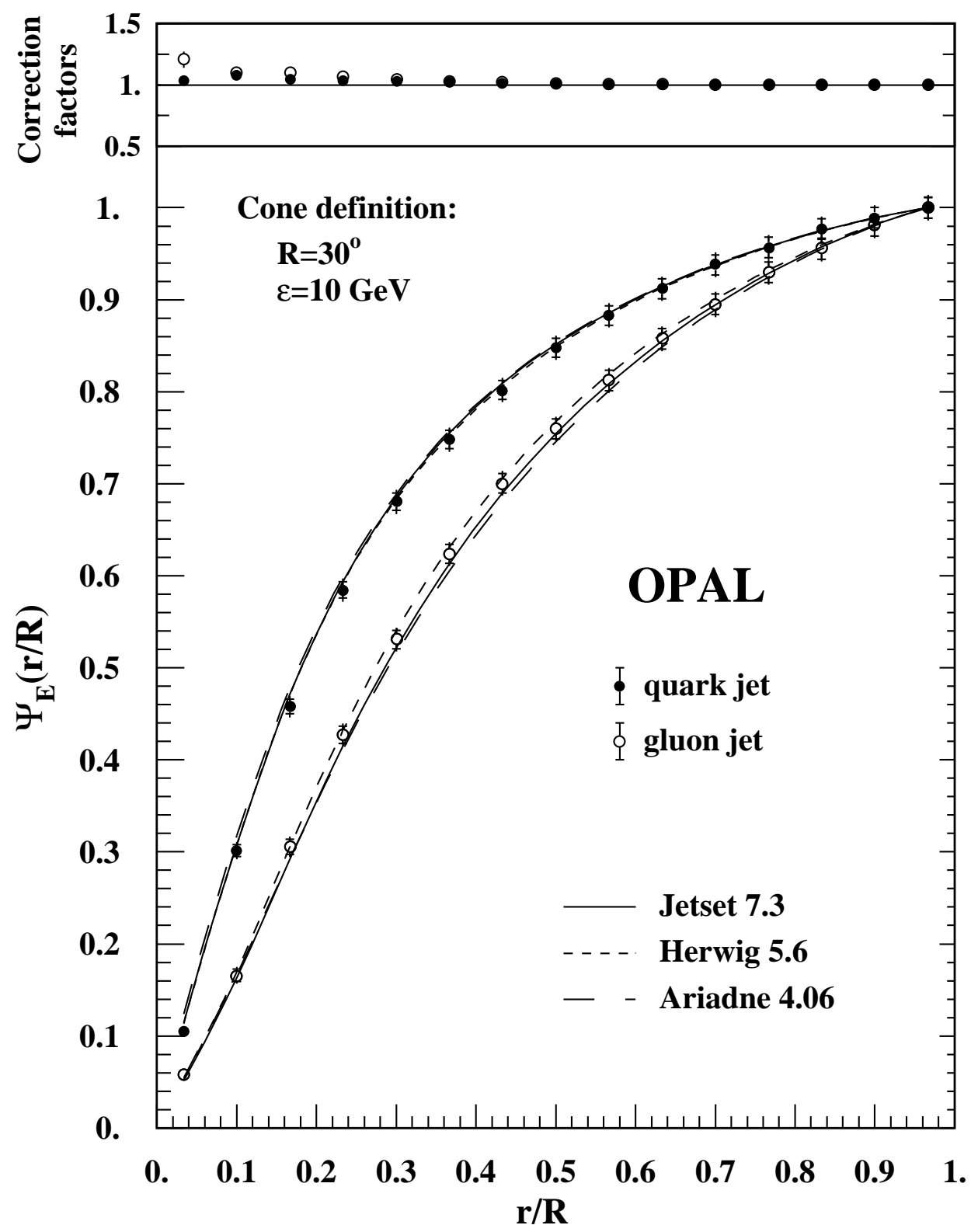

Figure 12: The integral energy profile of gluon and quark jets defined using the cone algorithm. The errors shown include both the statistical and systematic terms and are correlated from bin to bin; the experimental statistical uncertainties are indicated by the small horizontal bars. These data are tabulated in Table 8. 


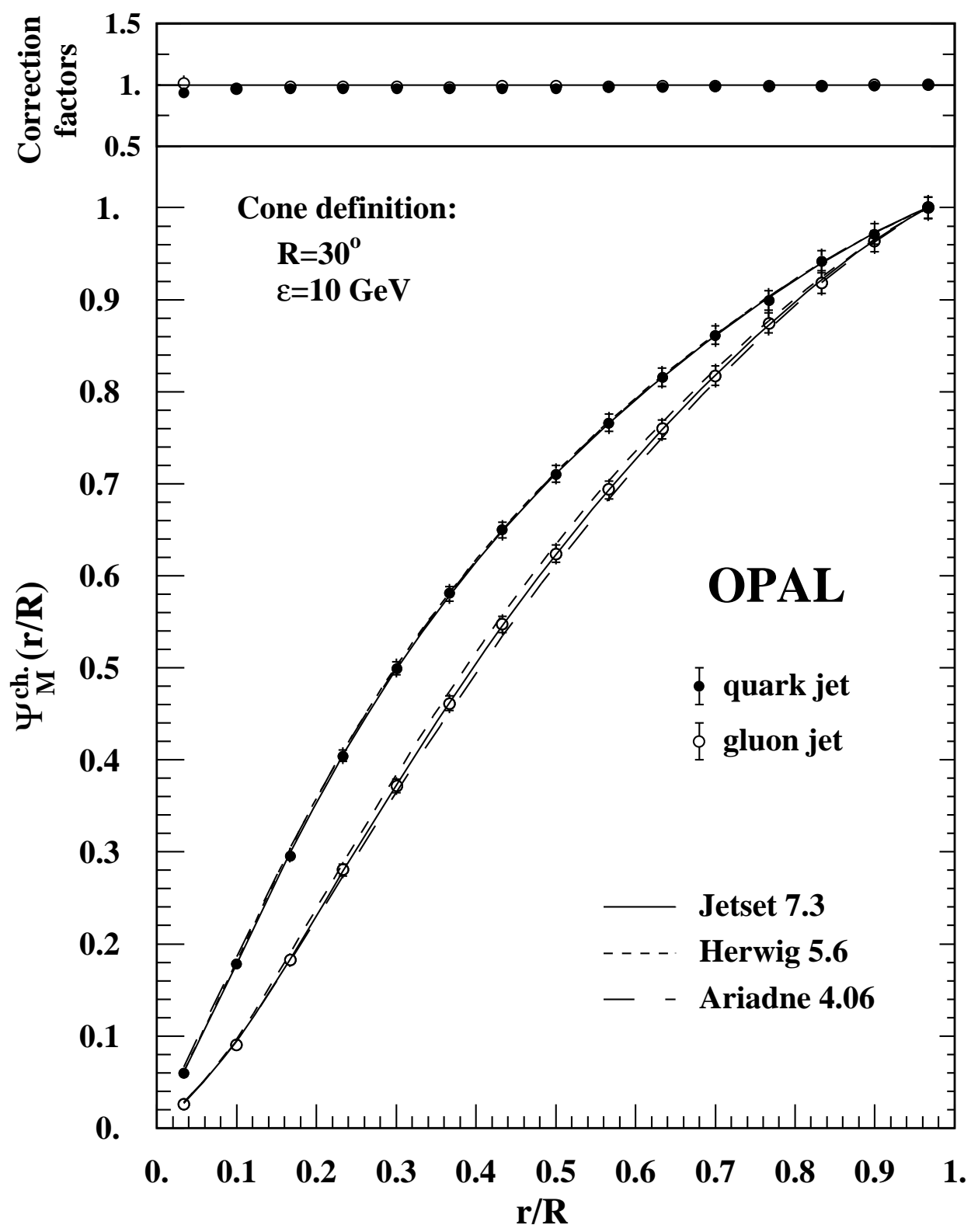

Figure 13: The integral charged particle multiplicity profile of gluon and quark jets defined using the cone algorithm. The errors shown include both the statistical and systematic terms and are correlated from bin to bin; the experimental statistical uncertainties are indicated by the small horizontal bars. These data are tabulated in Table 9. 


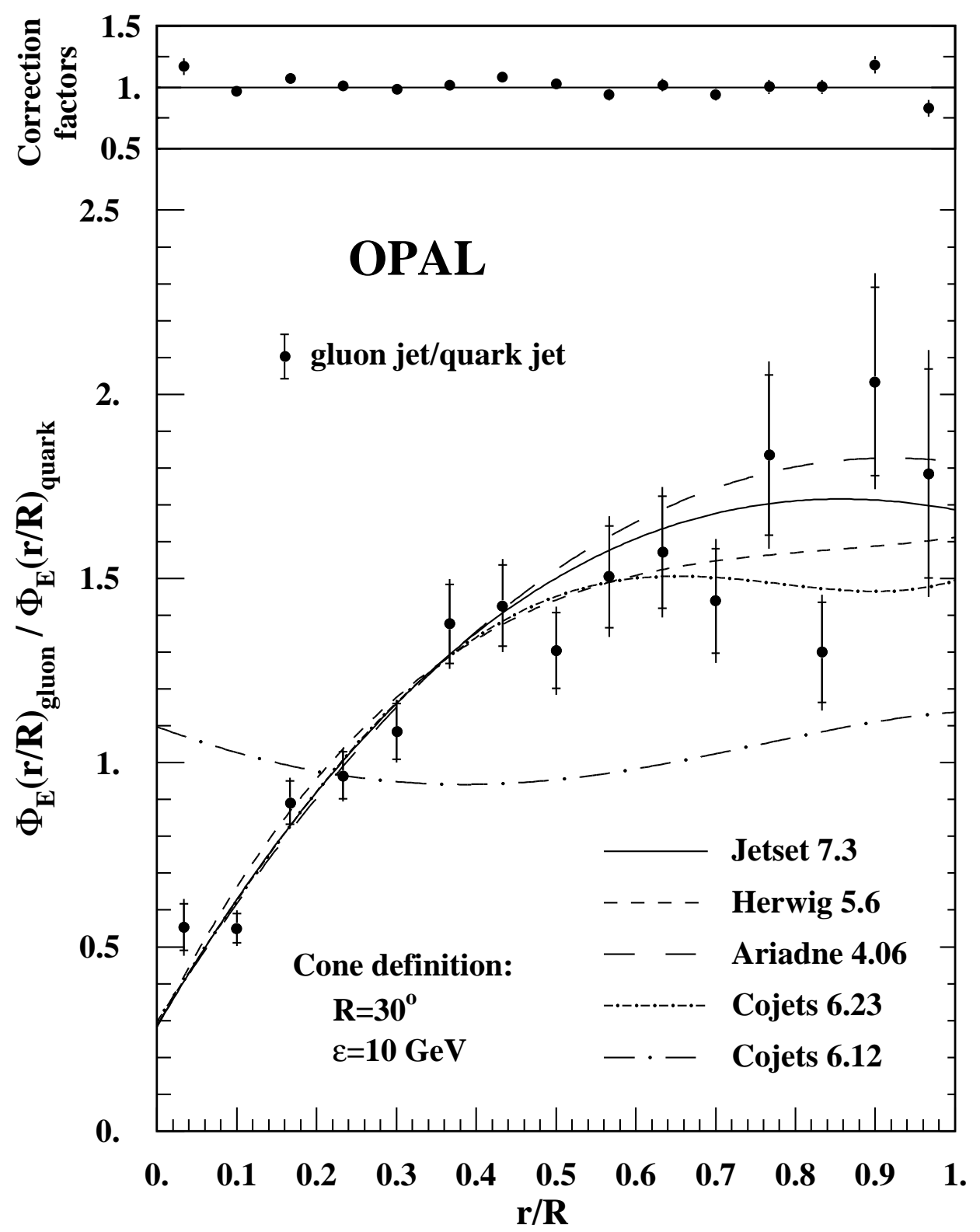

Figure 14: The ratio of the distributions shown in Fig. 10. The errors shown include both the statistical and systematic terms; the experimental statistical uncertainties are indicated by the small horizontal bars. These data are tabulated in Table 6 . 


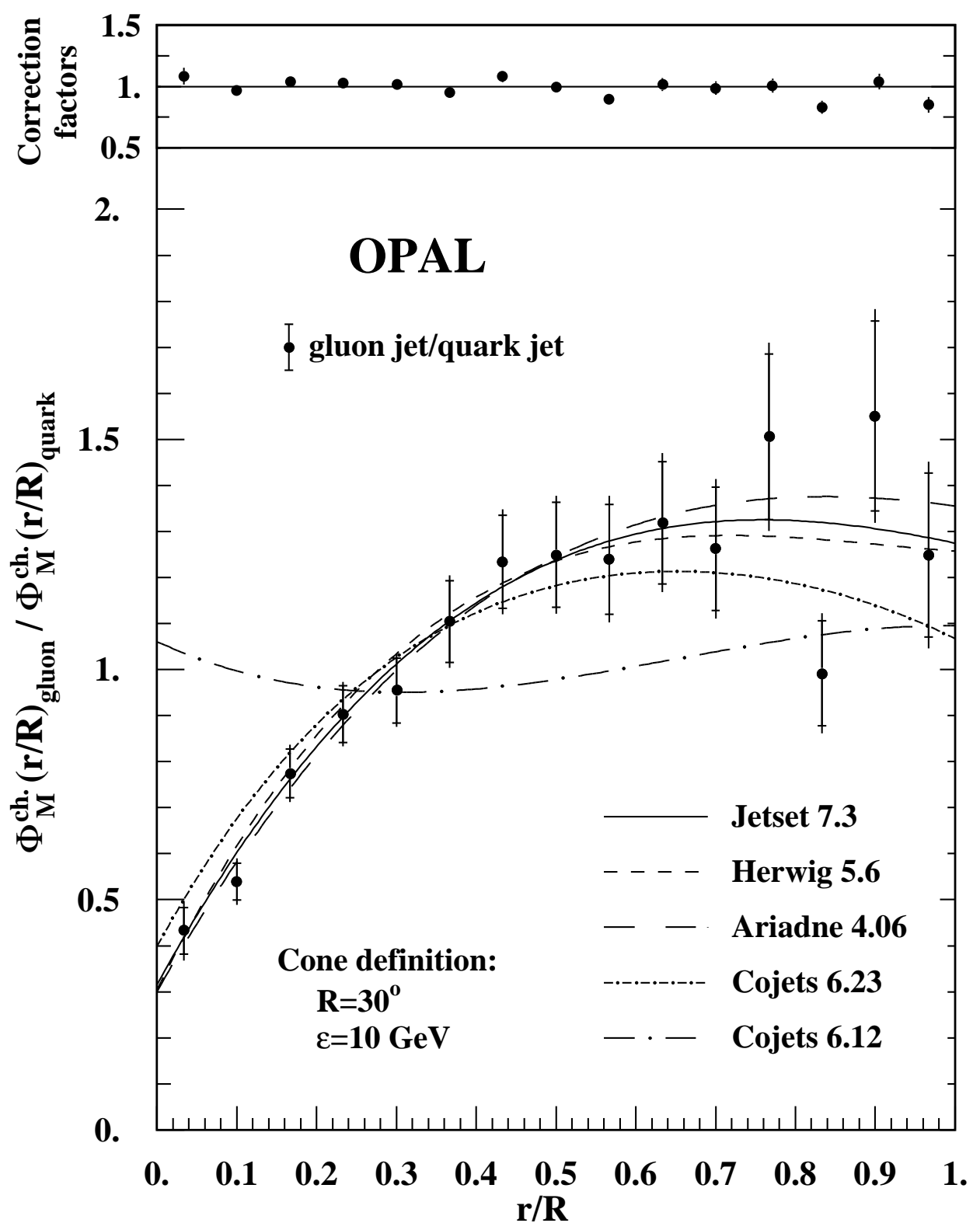

Figure 15: The ratio of the distributions shown in Fig. 11. The errors shown include both the statistical and systematic terms; the experimental statistical uncertainties are indicated by the small horizontal bars. These data are tabulated in Table 7. 

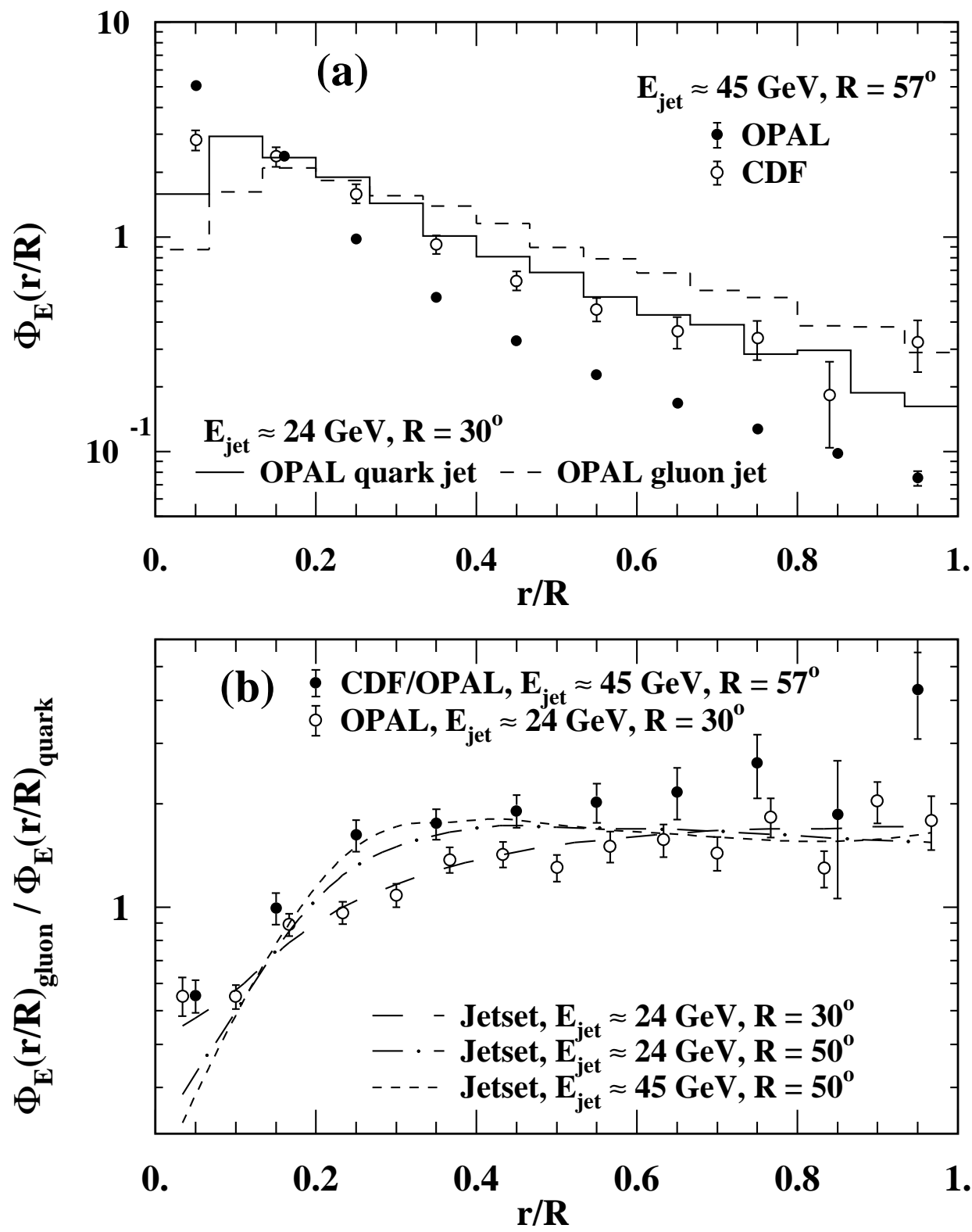

Figure 16: (a) The differential energy profiles of $45 \mathrm{GeV}$ jets measured by OPAL and CDF, using the cone jet finder, compared to the corresponding measurements for $24 \mathrm{GeV}$ jets presented in this paper. (b) Ratios of the data shown in (a); the predictions of the Jetset Monte Carlo for $24 \mathrm{GeV}$ jets with two choices for the cone size $R$, and for $45 \mathrm{GeV}$ jets with $R=50^{\circ}$, are also shown. 


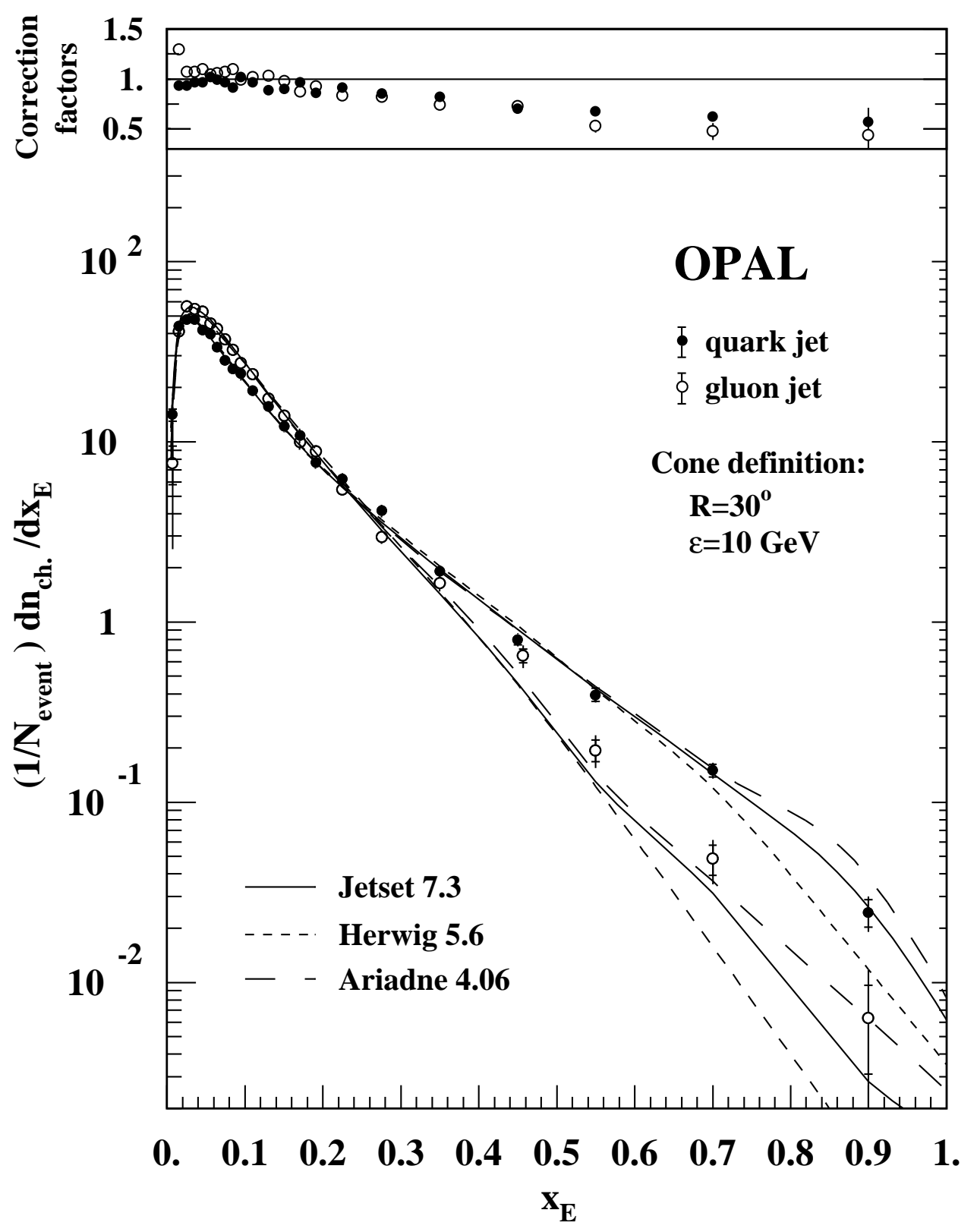

Figure 17: Charged particle fragmentation functions of quark and gluon jets defined using the cone jet finder. The errors shown include both the statistical and systematic terms; the experimental statistical uncertainties are indicated by the small horizontal bars. These data are tabulated in Table 10. 


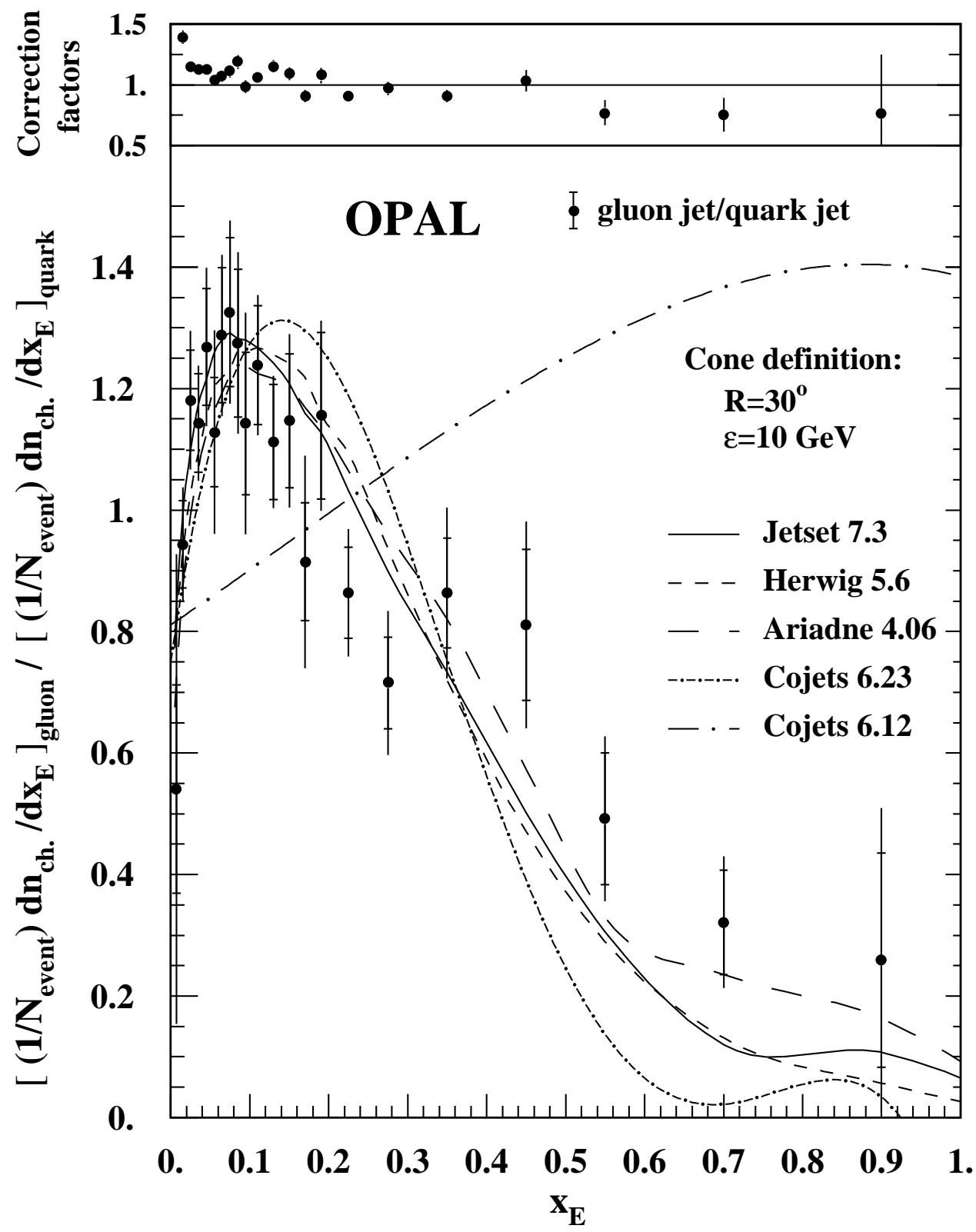

Figure 18: The ratio of the distributions shown in Fig. 17. The errors shown include both the statistical and systematic terms; the experimental statistical uncertainties are indicated by the small horizontal bars. These data are tabulated in Table 10. 

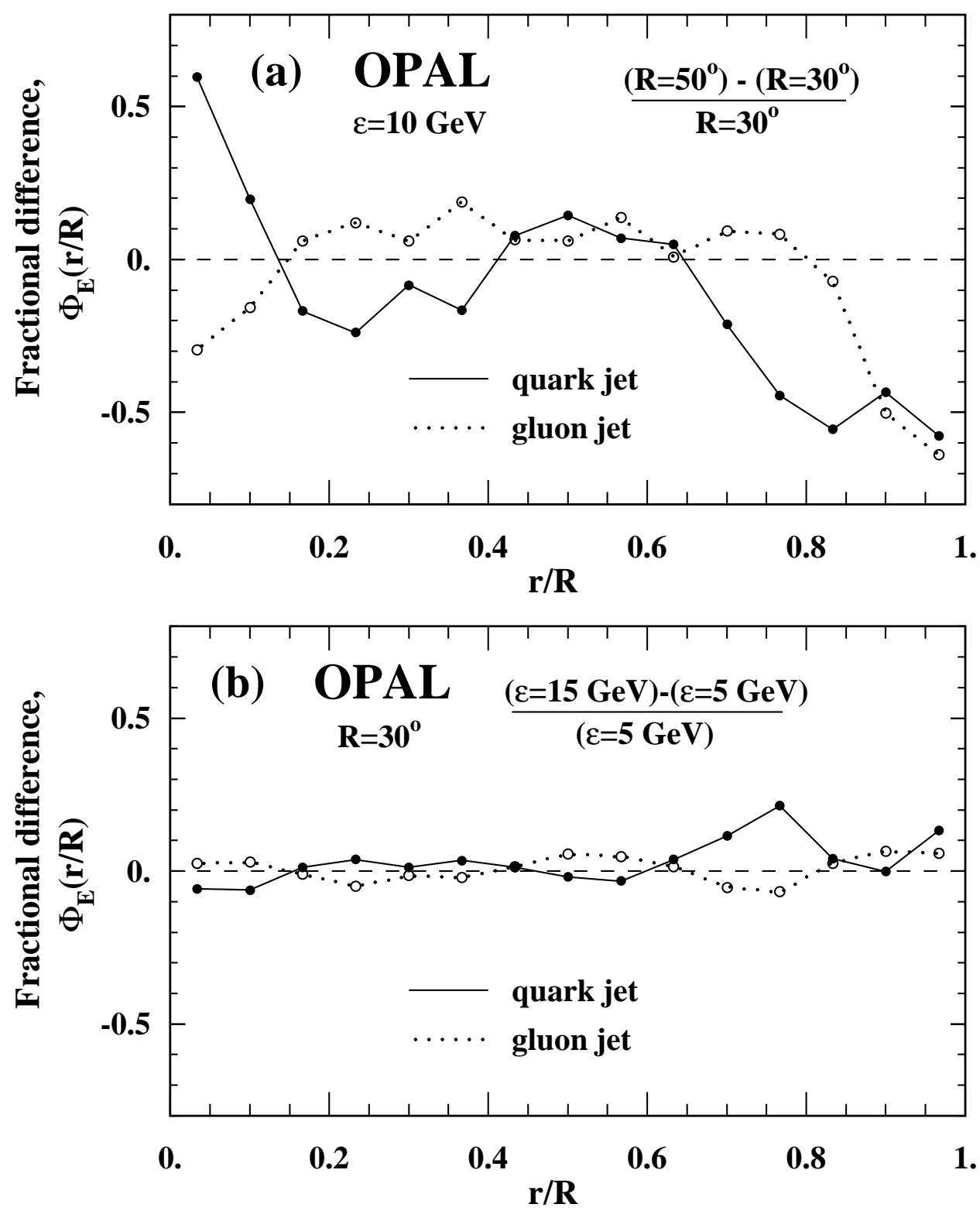

Figure 19: Fractional difference between the differential energy profile distributions of quark and gluon jets using (a) $R=50^{\circ}$ and $R=30^{\circ}$, with $\epsilon=10 \mathrm{GeV}$, and (b) $\epsilon=15 \mathrm{GeV}$ and $\epsilon=5 \mathrm{GeV}$, with $R=30^{\circ}$, using the cone jet finder. The point to point fluctuations in the curves indicate the level of statistical uncertainty. 


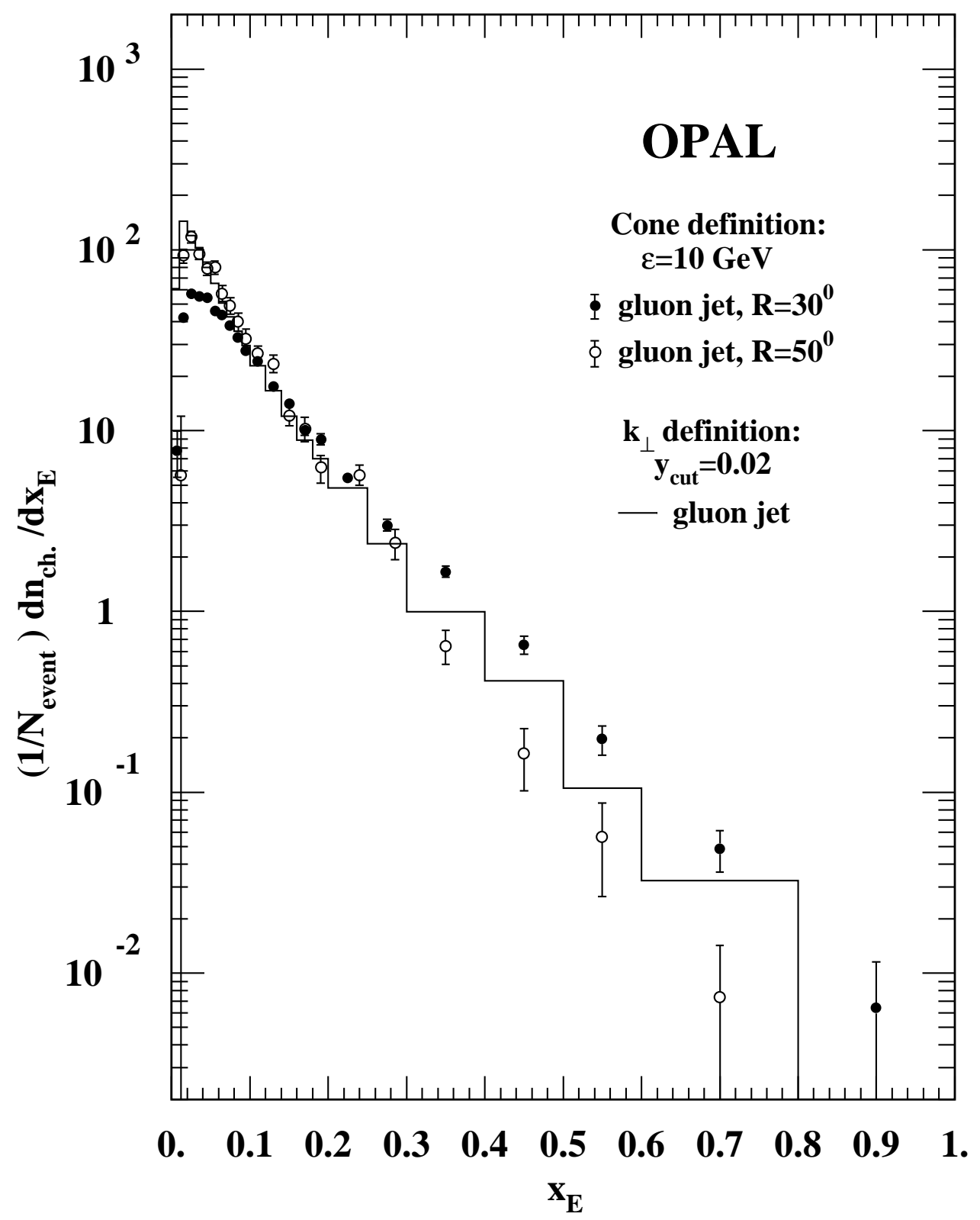

Figure 20: The gluon jet charged particle fragmentation function found using the cone jet finder with different cone sizes $R$ and $\epsilon=10 \mathrm{GeV}$, compared to that found using the $k_{\perp}$ jet finder with $y_{c u t}=0.02$. The uncertainties shown are the statistical terms from the experiment and detector correction factors, added in quadrature. 


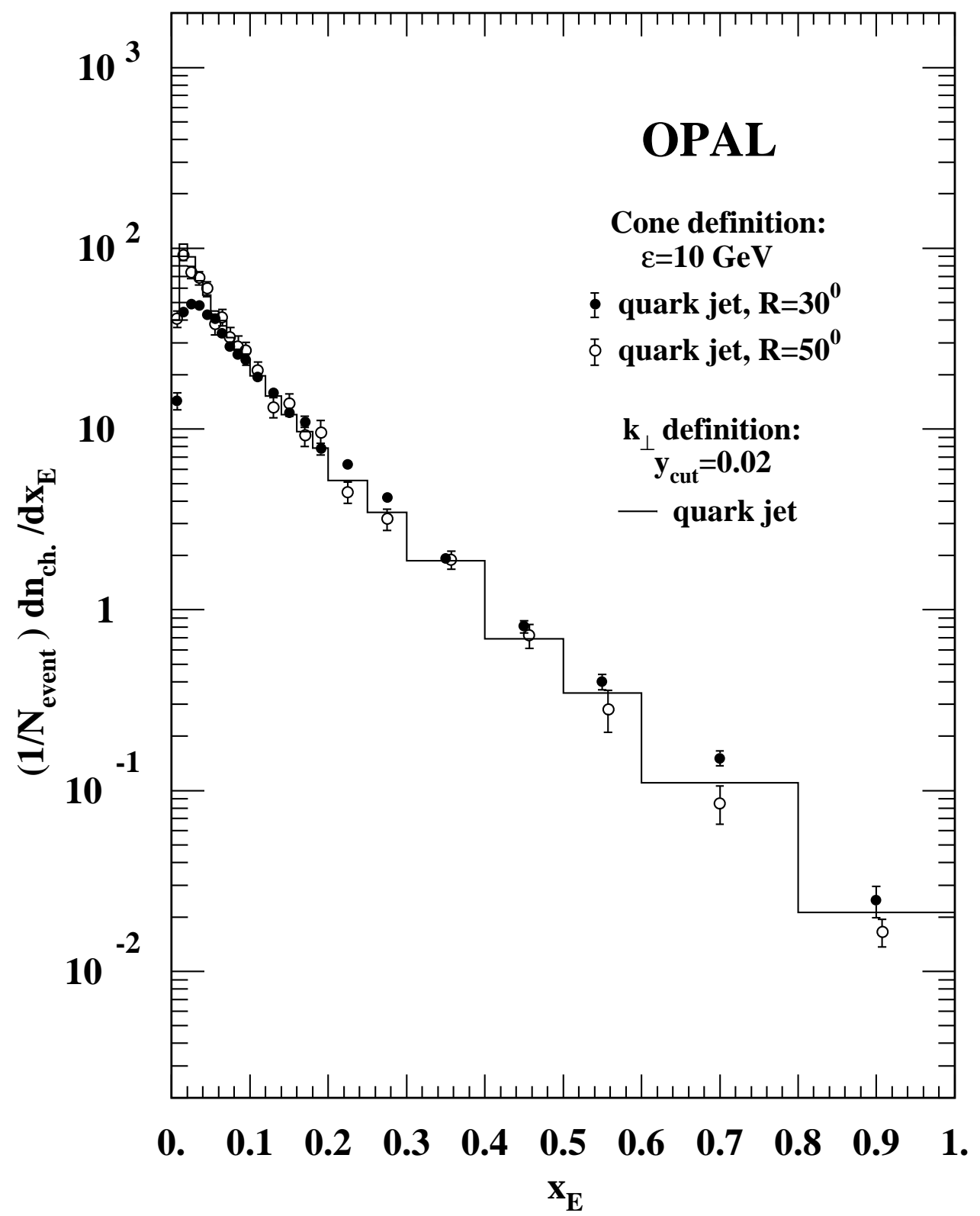

Figure 21: The quark jet charged particle fragmentation function found using the cone jet finder with different cone sizes $R$ and $\epsilon=10 \mathrm{GeV}$, compared to that found using the $k_{\perp}$ jet finder with $y_{c u t}=0.02$. The uncertainties shown are the statistical terms from the experiment and detector correction factors, added in quadrature. 

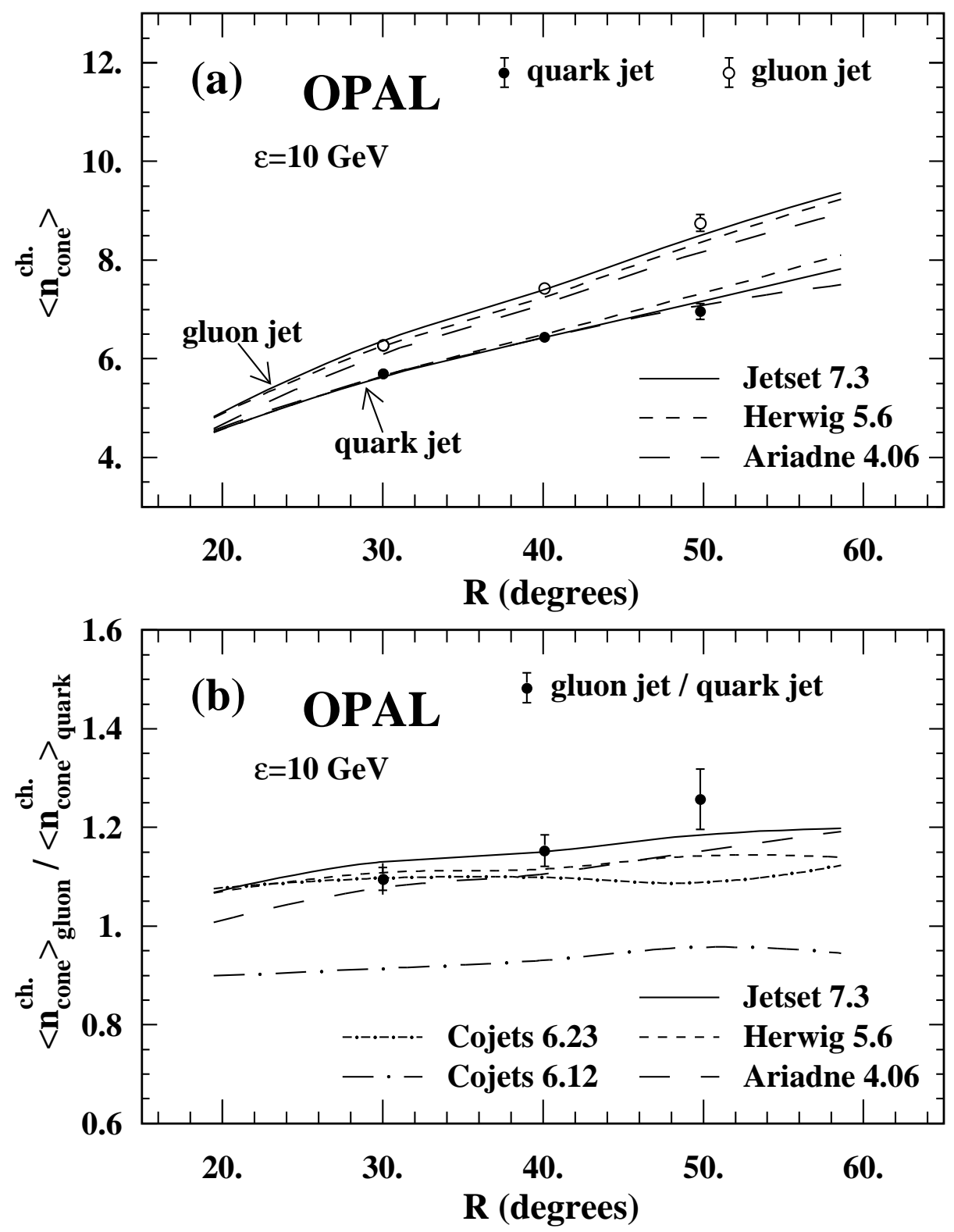

Figure 22: (a) Mean charged multiplicity values of gluon and quark jets defined using the cone jet finder and different cone sizes $R$, with $\epsilon=10 \mathrm{GeV}$. (b) Ratio of the gluon to quark jet mean charged multiplicity values as a function of $R$. For the results based on $R=30^{\circ}$, the total errors including statistical and systematic terms are shown; the size of the experimental statistical uncertainties are indicated by the small horizontal bars. For the other $R$ values, the uncertainties shown are the statistical terms from the experiment and detector correction factors, added in quadrature. These data are tabulated in Table 11. 


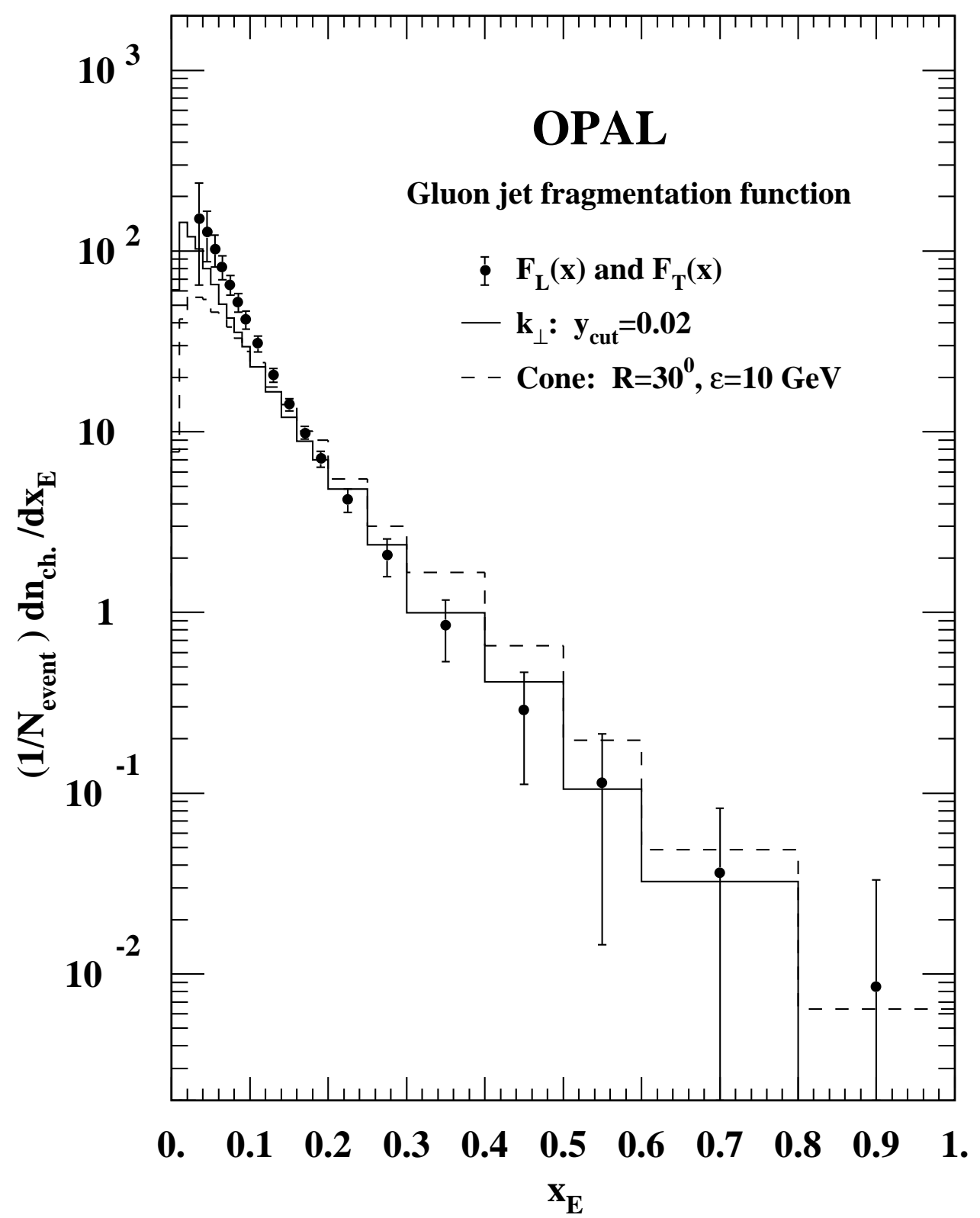

Figure 23: The gluon jet charged particle fragmentation function from [8], compared to that found using the $k_{\perp}$ and cone jet finders with their standard parameter values. 